OVERGENERAL AUTOBIOGRAPHICAL MEMORY AND PSYCHOPATHOLOGY IN ADOLESCENCE

BY

\title{
CHARLOTTE GUTENBRUNNER
}

\author{
A thesis \\ submitted to the Victoria University of Wellington \\ in fulfilment of the requirements for the degree of \\ Doctor of Philosophy \\ in Psychology
}

Victoria University of Wellington

2018 



\begin{abstract}
Overgeneral autobiographical memory (OGM) - the tendency to report more general event memories when instructed to report specific past events - has been implicated in the development and maintenance of poor psychological functioning (Sumner, Griffith, \& Mineka, 2010). One significant limitation of the OGM literature is that few studies have investigated associations between this memory bias and psychopathology in young people (Hitchcock, Nixon, \& Weber, 2014a). Delineating associations between OGM and psychological functioning in adolescence can be argued particularly important, however, as symptoms of psychopathology increase steeply (Cicchetti \& Toth, 1998). Specifically, longitudinal research with community youth is needed to clarify associations between OGM and psychological functioning before the onset of psychopathology. Accordingly, this thesis addressed three important gaps in the literature.

In the first study, we extend the field by testing whether OGM represents a marker of vulnerability for psychopathology (depression and anxiety) in community youth $(N=269)$ across three annual assessment points. Across the entire sample, OGM did not predict symptoms of depression or anxiety. For youth who engage in higher levels of rumination, OGM predicted increases in anxiety symptoms, but only across a single time lag. These findings demonstrate that OGM does not represent a risk factor for emerging psychopathology in community youth. Preliminary evidence suggests that OGM may interact with rumination to influence anxiety symptoms under some conditions.

The second study represents the first to test the predominant model of OGM- the
\end{abstract} CaR-FA-X model (Williams et al., 2007) — in its entirety and across four annual assessment points in community adolescents $(N=323)$. This theoretical account purports that three cognitive vulnerabilities (increased rumination and avoidance, and reduced executive control) foster OGM. Overall, findings from Study 2 suggest that the CaR-FA-X model has limited applicability in community youth. Increased avoidance predicted OGM, but this effect was limited to the final time lag and only emerged in the context of elevated longitudinal depression levels. Perhaps OGM represents a form of cognitive avoidance in youth when low mood persists for extended periods of time.

In the third, and final, study we extend the literature by investigating associations between OGM and event-specific memory detail in a sample of community youth $(N=96)$. We also examined similarities and differences in how these two facets of autobiographical recollection associate with symptoms of depression, anxiety, and rumination across three annual assessment points. We found that youth who reported more specific memories did not 
report more detailed event recollections. Moreover, memory specificity and detail embedded in specific memories did not shed light on changes in psychological functioning. Rather, we found transient evidence of decreases in memory specificity and detail as a function of higher anxiety and rumination. As effects were inconsistent across time, conclusions can only be made cautiously, however.

This thesis advances the field in several ways. The overarching patterns of findings across the three studies highlight that OGM does not represent an index of poor psychological functioning in community adolescents. The memory phenomenon did not predict increases in symptoms of depression or anxiety, nor did the three cognitive vulnerabilities that make up the CaR-FA-X model explain significant change in OGM. Moreover, OGM was not associated with biases in reporting of memory detail. Transient associations between OGM and psychological difficulties were found, but only in the context of heightened risk for psychopathology. Perhaps this style of remembering the past only has negative consequences for well-being in adolescence when it occurs alongside other cognitive and emotional problems. 


\section{Acknowledgements}

This research could not have happened without the generous time and effort from the young people who participated in this longitudinal research project. The information they provided year after year gave me a small glimpse into their most personal inner life, and I feel very privileged that I was allowed to use it for my research. I would also like to say thank you to participants' parents, teachers, principals and other school staff. Without their continued support and hospitality, this project would not have survived.

Next, I would like to say thank you to my primary supervisor Professor Karen Salmon. From the day I first stepped into her office as an undergraduate student, she was supportive, never lost faith in my abilities, and encouraged me to push myself out of my comfort zone. Karen, thank you for your endless wisdom, expertise and patience. I would also like to say thank you to my secondary supervisor Professor Paul Jose, for his constant support and enthusiasm for statistics. My current statistical knowledge is a credit to his ability to explain complex analyses in plain English. I could not have asked for better mentors.

I am grateful for the various forms of financial support I received over the years (Victoria University of Wellington Doctoral and submission scholarships, FSRG travel grants, NZCCP travel grant, Maurice and Phyllis Paykel Trust travel grant). Also, thank you to the Royal Society of New Zealand who funded parts of this project.

Thank you to the many research fellows, assistants and students who were a part of the Salmon lab over the years. You helped with collecting, entering and coding of the data, and, most importantly, became good friends. You made this journey so much more fun and memorable!

I am grateful for the support of my friends. You listened to me go on about the wonders of statistics (I hope I convinced at least one of you!), made me laugh when I was down and bought me food when I had no time to cook. A special thank you to Lauren Bryce and Tim Ganly. Thank you for being two of the most supportive, caring and funny people I know. Although we will no longer see each other on a daily basis in the lab, you have become friends for a lifetime.

Finally, I dedicate this thesis to my family. Mum, Dad and Hannes, I could have not got to the point of finishing without your endless love. You have always believed in me, motivated me to keep going when I struggled, and acted as a constant reminder of what is important in life. Thank you for supporting me emotionally and financially when I needed it. To my Omis, I know you would both be proud. 


\section{Table of Contents}

Abstract

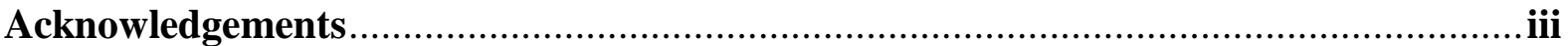

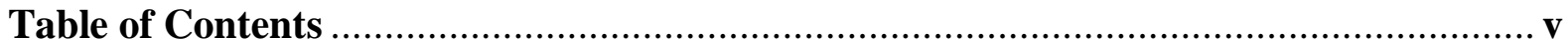

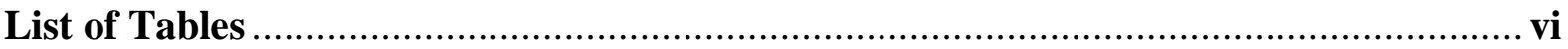

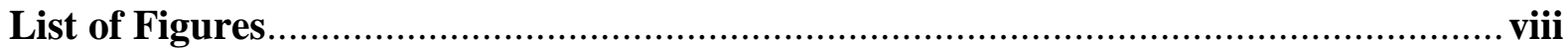

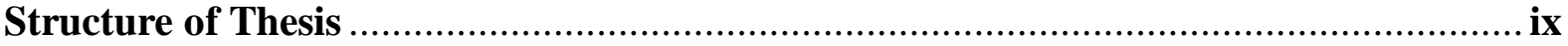

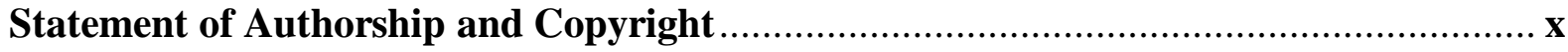

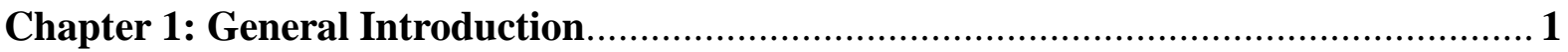

Overgeneral Autobiographical Memory (OGM) ........................................................ 1

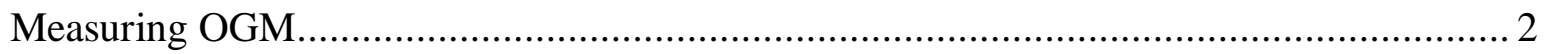

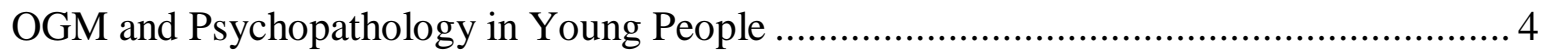

Theory of Overgeneral Autobiographical Memory....................................................... 7

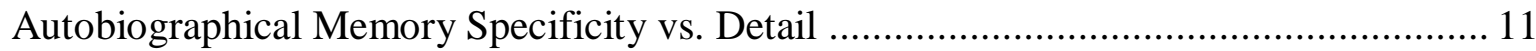

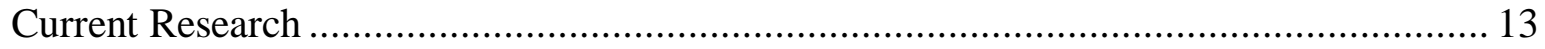

Chapter 2: Do Overgeneral Autobiographical Memories Predict Increased

Psychopathological Symptoms in Community Youth? A 3-Year Longitudinal

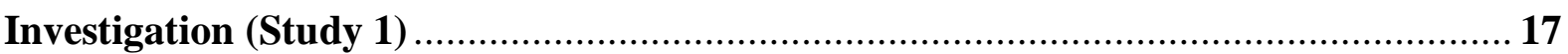

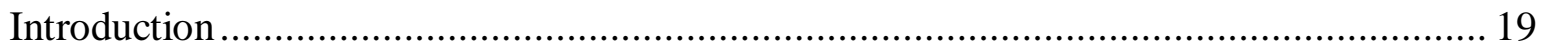

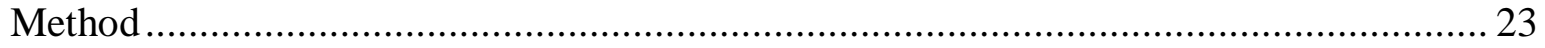

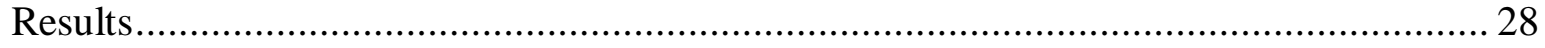

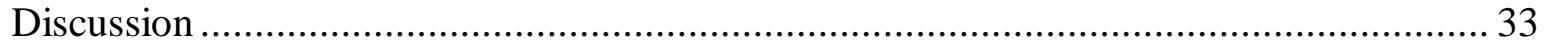

Chapter 3: What Predicts Overgeneral Autobiographical Memory in Youth? Testing the CaR-FA-X Model Longitudinally in Adolescents (Study 2) ................................. 39

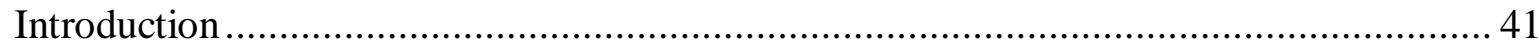

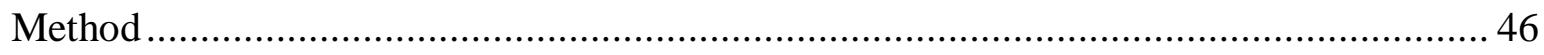

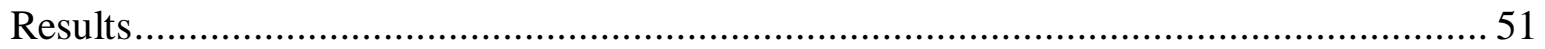

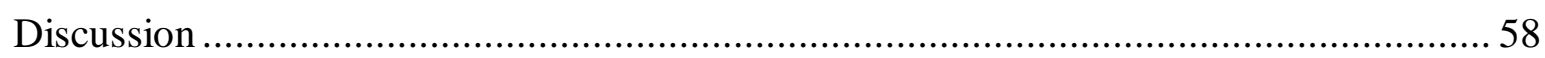




\section{Chapter 4: Autobiographical Memory Specificity and Detail: Investigating}

Introduction

Method .71

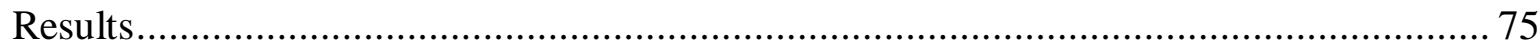

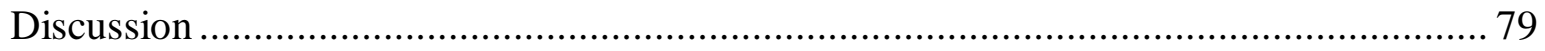

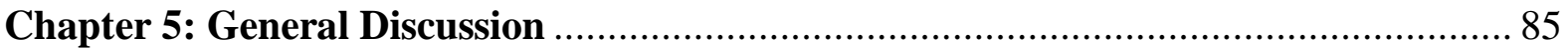

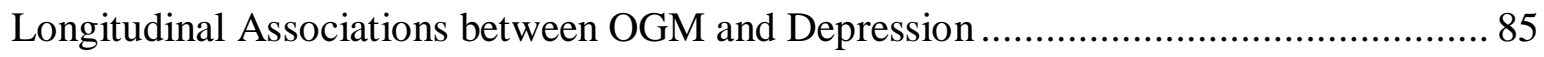

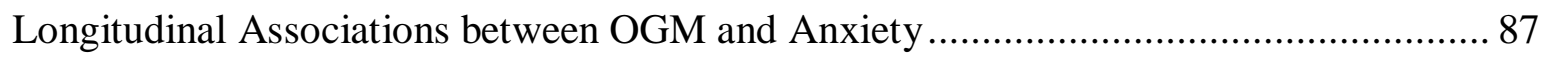

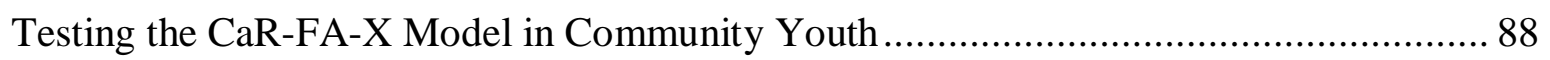

Episodic Detail: Associations with Memory Specificity and Psychopathology ................ 91

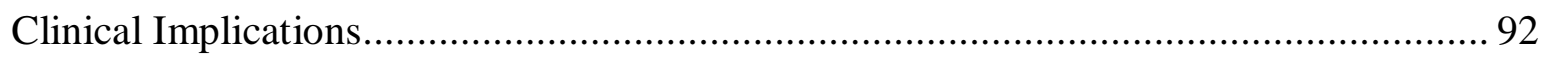

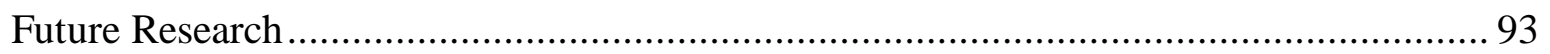

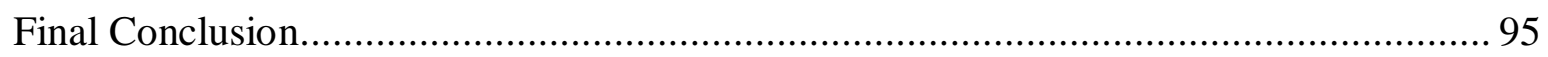

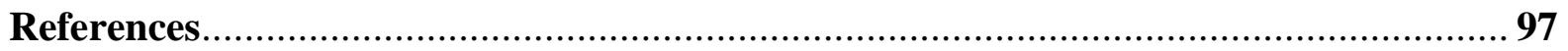

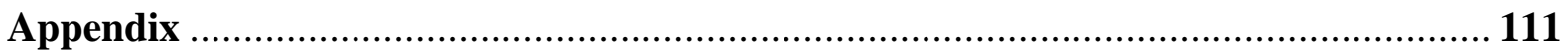

Appendix A: Autobiographical Memory Test (AMT) booklet with sample memory

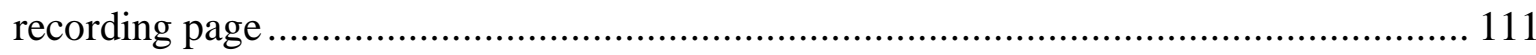

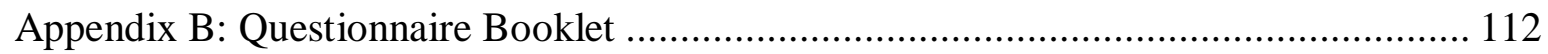

Appendix C: Autobiographical Memory Coding Scheme.......................................... 119

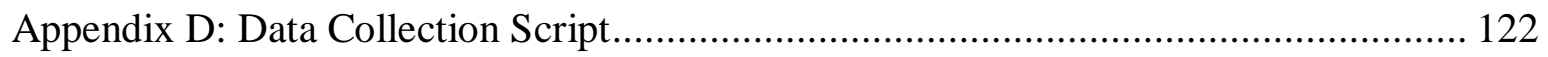

Appendix E: Episodic Detail Coding Scheme ..................................................... 124 


\section{List of Tables}

Table 1 Study 1: Correlations Between Study Variables $(N=269)$.................................. 30

Table 2 Study 1: Means and Standard Deviations.......................................................... 31

Table 3 Study 2: Descriptive Statistics of the Study Variables $(N=323)$........................... 52

Table 4 Study 2: Pearson's Correlations Between CaR-FA-X Model Factors and Primary Memory Indices

Table 5 Study 2: Descriptive Statistics of the Study Variables for Low $(N=176)$ and Medium/High $(N=147)$ Depression Growth Subgroups. 55

Table 6 Study 2: Standardized Beta Coefficients and Bias-corrected 99\% Bootstrapped Confidence Intervals for Avoidance (T3) Predicting OGM Proportions (T4) Across Entire Sample and Depression Growth Subgroups .....

Table 7 Study 3: Descriptive Statistics of the Study Variables for Low and Medium/High Rumination Growth Subgroups 76

Table 8 Study 3: Pearson Correlations between Memory Dimensions and Other Key Variables

Table 9 Study 3: Correlations between Memory Dimensions 78 


\section{List of Figures}

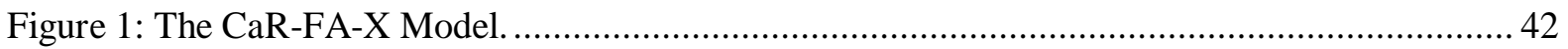




\section{Structure of Thesis}

This thesis is composed of a general introductory chapter (Chapter 1), one published article (Chapter 2), one article that is currently under review (Chapter 3), one article that has been submitted for publication (Chapter 4), and a general discussion chapter (Chapter 5). Repetition of information may occur across Chapter 1 and the introductions of the three empirical chapters. To maximize the flow of the thesis, I have included transition pages that link the articles and discuss how they fit into the thesis as a whole. Although I was the lead author, I will use the term "we" when discussing material related to these three co-authored articles throughout the thesis.

\section{Chapter $\mathbf{2}$ is the accepted version of the following article:}

Gutenbrunner, C., Salmon, K., \& Jose, P. E. (2017). Do overgeneral autobiographical memories predict increased psychopathological symptoms in community youth? A 3year longitudinal investigation. Journal of Abnormal Child Psychology. Advance online publication. doi:10.1007/s10802-017-0278-5

\section{Chapter 3 is composed of a manuscript that is currently under review with} Development and Psychopathology:

Gutenbrunner, C., Salmon, K., \& Jose, P. E. (2017). What predicts overgeneral memory in youth? Testing the CaR-FA-X model longitudinally in community adolescents. Manuscript under review.

Chapter 4 is composed of a manuscript that has been submitted to Memory:

Gutenbrunner, C., Salmon, K., \& Jose, P. E. (2017). Episodic specificity and detail: Investigating longitudinal associations with psychopathology in youth. Manuscript submitted for publication. 


\section{Statement of Authorship and Copyright}

I am the primary (lead) author on the co-authored articles presented in this thesis. I collected and managed the data, developed the research questions, conducted and interpreted the analyses, and wrote the first drafts. Karen Salmon and Paul Jose were involved in discussions about, and conceptualisation of, the study design and research questions, and provided critical revisions.

With regard to copyrighted published material (Chapter 2, pages 18-37), I confirm that a licence to reproduce this work has been granted by Springer Nature:

Reprinted by permission from Springer Customer Service Centre GmbH: Springer Nature, JOURNAL OF ABNORMAL CHILD PSYCHOLOGY, DO OVERGENERAL AUTOBIOGRAPHICAL MEMORIES PREDICT INCREASES IN PSYCHOPATHOLOGICAL SYMPTOMS IN COMMUNITY YOUTH? A 3-YEAR LONGITUDINAL INVESTIGATION, CHARLOTTE GUTENBRUNNER, KAREN SALMON, PAUL JOSE, (C) 2017, advance online publication, 3 August 2017, doi:10.1007/s10802-017-0278-5. 


\section{Chapter 1}

\section{General Introduction}

\section{Overgeneral Autobiographical Memory (OGM)}

Autobiographical memory - memory of personally experienced events — defines our life story, provides us with a sense of identity in relation to others and informs our everyday behaviour (Bluck, Alea, Habermas \& Rubin, 2005; Conway, 2005; Nelson \& Fivush, 2004). Not surprisingly, due to the deeply personal and self-relevant nature of autobiographical memories, the ability to remember specific events from our past has been argued central to psychological well-being (Conway \& Pleydell-Pearce, 2000; Williams et al., 2007). So what if an individual finds it difficult to recall events from their past, how might this affect their functioning? This question has been the focus of an area of research which I will refer to as the overgeneral autobiographical memory literature. Overgeneral autobiographical memory (OGM) is the tendency to report general memories when instructed to retrieve specific event memories in response to cue words (Williams et al., 2007). For example, rather than reporting a specific memory such as "when my sister ripped my clothes on Monday" in response to the cue word angry, an individual might report "when I fight with my sister", which refers to a collection of similar events, or "when my sister hid my clothes for a week", which refers to an event that lasted for an extended period of time.

The finding that recent suicide attempters retrieved more general memories on a cueing-paradigm than individuals who reported lower emotional distress sparked initial interest in this phenomenon (Williams \& Broadbent, 1986). The authors proposed that people with a mood disturbance may experience difficulties with accessing specific autobiographical memories. Since this first conceptualisation of increased general memory retrieval as a memory processing impairment in suicidal adults, numerous studies have replicated, and extended, this seminal research. Three important conclusions can be drawn from their collective findings. First, the tendency to recall OGMs is not limited to suicidally depressed patients; robust cross-sectional associations between OGM and a range of psychological presentations have been reported, in particular depression and PTSD (Williams et al., 2007). Second, OGM is implicated in the maintenance of depression and post-traumatic stress, and predicts poorer prognosis (see Moore \& Zoellner, 2007, for a review; Sumner et al., 2010, for a meta-anlaysis). Third, cross-sectional and longitudinal associations between OGM and psychopathology akin to those in adults are also found in children and adolescents (e.g., Hamlat et al., 2015; see Hitchcock et al., 2014a, for a review). These findings have led to the 
conceptualisation of OGM as a cognitive vulnerability marker of psychopathology (e.g., Williams et al., 2007). Before discussing research evidence and implications of associations between OGM and psychopathology in children and adolescents in more detail, I will now provide an overview of how OGM is measured and conceptualised.

\section{Measuring OGM}

The phenomenon of OGM is typically assessed with the Autobiographical Memory Test (AMT; Williams \& Broadbent, 1986). The AMT is a cueing-paradigm, and, as such, requires individuals to generate specific event memories from their past in response to external stimuli. Participants are: 1) instructed to report a specific memory that each cue word reminds them of; 2) provided with examples of correct and incorrect answers; and 3) given the opportunity to complete practice trials. Although the original AMT requires generation of a spoken response, written versions of the AMT have also been used (e.g., Raes, Hermans, de Decker, Eelens, \& Williams, 2003). When completing the test, participants are then typically required to retrieve memories to positive and negative emotion-focused cue words (e.g., sad and excited) within a specified time interval (e.g., 30-60 seconds). While the majority of research on OGM adopts the AMT as a measure of memory specificity, task characteristics (e.g., number of cue words, time interval and mode of administration) can vary (see Griffith et al., 2012). Although the AMT was originally developed for use with adults, it has since been adopted successfully by researchers investigating OGM in children and adolescents (e.g., Heron et al., 2012; Nuttall, Valentino, Comas, McNeil, \& Stey, 2014; Raes, Verstraeten, Bijttebier, Vasey, \& Dalgleish, 2010).

One limitation of the traditional AMT is that it tends to elicit a restricted range of OGMs in non-clinical populations compared to clinical populations. Past research suggests that the test instructions may reduce the test's sensitivity to OGM in higher-functioning individuals, giving rise to ceiling effects (Raes, Hermans, Williams, \& Eelen, 2007). Developed for use with suicidal and depressed patients, the test instructions strongly emphasize the goal of retrieving specific memories. It is argued that although a person may engage in higher overgeneral remembering in everyday life, under more stringent testing conditions, wherein which the task goal is repeatedly reinforced, they may be able to overcome such a tendency. To address this limitation, Debeer, Hermans and Raes (2009) developed the "Minimal Instructions AMT" (Mi-AMT), in which participants are still asked to retrieve real memories, but not instructed that events should have occurred on a particular day. Examples and practice items are also not provided. Research highlights increased sensitivity of the Mi-AMT to individual differences in OGM across non-clinical 
populations - in the form of greater variability in test scores — warranting use of this alternative test with higher functioning individuals (Debeer et al., 2009; Griffith et al., 2009).

The memories generated by individuals on the AMT, as well as the Mi-AMT, are scored as either specific or non-specific (Williams \& Broadbent, 1986). A memory is coded as specific when it refers to an event that occurred on a particular day (e.g., "when I went biking on Saturday"). As such, specific responses are temporally specific, referring to unique events that happened at a particular time. Non-specific memories refer to responses that are not specific in time: categories of similar events (e.g., "when I go swimming"), extended periods of time (e.g., "when I went to Fiji"), semantic associations of the cue word (e.g., "bed" in response to relaxed) and omissions. Across past research, there is vast variability in how OGM was operationalised. For example, in studies with youth, OGM indices represented number of specific memories (e.g., Rawal \& Rice, 2012a and b), number of categoric memories (e.g., Raes, Verstraeten, Bijttebier, Vasey \& Dalgleish, 2010), and sum of categoric and extended memories (e.g., Hitchcock, Nixon, \& Weber, 2014b). Moreover, while some research counted instances of memory types (e.g., Stokes, Dritschel \& Bekerian, 2004), other research calculated proportions of memory types out of the total number of memories reported (e.g., Park, Goodyer \& Teasdale, 2004). It is important to acknowledge this variability in operationalisation of OGM as a limitation of past research that places constraints on generalizability of findings.

Notably, regardless of memory index adopted, the terms overgeneral memory and reduced memory specificity are used interchangeably in research to refer to OGM. However, measures of overgenerality, in the form of categoric and extended memories, and specificity do not necessarily represent the same phenomenon. That is, while two people may report the same low number of specific memories, remaining non-specific memories may be overgeneral memories for one individual, but consist of omissions and semantic associates for the other. As such, an individual need not be overgeneral in order to be non-specific. In support of the notion that these indices do not necessarily represent the same underlying construct are findings of differences in how they associate with other variables (e.g., Hermans, Defranc, Raes, Williams \& Eelen, 2005). Further complicating the picture is inconsistency in computation of overgenerality and specificity indices, as discussed in the previous paragraph. In this thesis, I will use the terms overgeneral memory and memory specificity, as well as the abbreviation $O G M$, to broadly refer to retrieval of fewer specific memories and retrieval of more overgeneral memories (categoric and/or extended). However, I aim to differentiate 
between the various conceptualisations of OGM when discussing past and current research findings.

\section{OGM and Psychopathology in Young People}

Since Williams and Broadbent's (1986) finding of OGM in suicidal adults, the majority of research on OGM and its relationship with psychopathology has been with adults. Of relevance to the current research-with its focus on OGM and development of psychopathology in youth - is the finding of a robust association between this style of remembering and depressive symptoms (see Sumner et al., 2010, for a meta-analysis). The conceptualisation of OGM as a vulnerability marker of depression was primarily driven by the finding that OGM not only concurrently relates to depressive symptoms, but also predicts increases in symptoms and more severe course of depressive disorder (Williams et al., 2007). If OGM predicts depression above and beyond pre-existing symptom levels, it is not merely an epiphenomenon of depression, but a risk factor that can indicate vulnerability. At this point, it is important to note that although investigation of associations between OGM and post-traumatic stress represents a major focus of this literature, it is beyond the scope of the current thesis and will only be included in discussion where deemed necessary (see Moore \& Zoellner, 2007; Ono, Devilly \& Shum, 2016, for reviews).

Depression. While research with children and adolescents is more limited, findings suggest that patterns of association between OGM and depressive symptoms are comparable to those found in adult populations (see Hitchcock et al., 2014a, for a review). For example, in terms of cross-sectional studies, higher levels of OGM are associated with higher depressive symptoms (e.g., Kuyken \& Dalgleish, 2011; Orbach, Lamb, Sternberg, Williams \& Dawoud-Noursi, 2001; Raes et al., 2010). It is important to note that the majority of crosssectional research investigated associations in populations at risk for future depression, however; that is, youth who are at risk of (e.g., Kuyken \& Dalgleish, 2011), or are currently experiencing (e.g., Park, Goodyer, \& Teasdale, 2002), depression. This poses a limitation as it is unclear whether OGM represents a predictor or product of early psychological difficulties. Longitudinal research is needed to clarify whether OGM represents a vulnerability marker of early depression.

Despite its important role in delineating temporal relationships between OGM and psychopathology, longitudinal research with youth is limited in number. Moreover, findings are mixed across clinical and non-clinical populations. Across the three studies with youth at risk for future depression, higher numbers of OGMs predicted increases in depressive symptoms in females at familial risk for depression (Rawal \& Rice, 2012b) and females who 
scored high on a depression screening measure at baseline (Hipwell, Sapotichne, Klostermann, Battista \& Keenan, 2011). Similarly, fewer specific memories predicted onset of a major depressive episode in youth with past major depression, but only when young people also reported concurrent high levels of interpersonal stress (Sumner et al., 2011).

Considering OGM's proposed role as a marker of vulnerability for depression, it is important to establish whether an overgeneral style of remembering precedes, and predicts, increases in early depressive symptoms. Although research has identified adolescence as critical for the onset of psychopathology, with prevalence rates of depression, in particular, rising steeply (Cicchetti \& Toth, 1998; Costello, Foley and Angold, 2006; Thapar, Collishaw, Pine \& Thapar, 2012), only three studies have so far investigated associations between OGM and depression longitudinally in non-clinical youth. The labels "non-clinical" and "community", in this thesis, refer to youth who were not recruited because of past, and/or current, elevated depression levels, or because they qualify as "high-risk" (e.g., genetic vulnerability). Two studies found that higher numbers of OGMs predicted increases in depressive symptoms in this population, but only in conjunction with reports of increased emotional abuse (Stange, Hamlat, Hamilton, Abramson \& Alloy, 2013) or elevated rumination and more stressful life events (Hamlat et al., 2015). In contrast, Crane et al. (2016) reported that OGM did not predict depressive symptoms at a 3-year follow-up.

Moreover, findings from autobiographical memory research outside the OGM literature highlight that retrieval of some general memories, when asked to report specific memories, is normative in young people (e.g., Wang \& Conway, 2004). Indeed, recall of repeated event memories can serve a social directive function and be associated with increased psychological well-being in young adults (Waters, 2014). Despite differences in mode of memory retrieval and population under investigation, these findings highlight that reporting of some non-specific memories is inevitable and may even serve adaptive functions under certain circumstances.

Together, findings point to the possibility that the association between OGM and increases in depressive symptoms during adolescence is moderated by other risk factors. For adolescents at increased risk of depression, in the form of familial risk or past/current elevated depression levels, OGM may increase susceptibility to depression growth over time. In community youth, the relationship between OGM and depressive symptoms appears more nuanced. That is, for adolescents who are not at known risk for depression, OGM seems to either interact with other cognitive vulnerabilities of psychopathology in predicting depression over time (e.g., rumination; Hamlat et al., 2015), or not predict significant change 
(Crane et al., 2016). In non-clinical youth, therefore, overgeneral remembering may render a young person more vulnerable to developing depression only under conditions of heightened emotional distress and increased engagement in other cognitive processing biases such as rumination. In this literature, rumination has typically been defined as a pervasive and passive form of responding to distress, wherein the person thinks about their negative experience, its causes and potential consequences, rather than facilitating more adaptive problem-solving strategies (Nolen-Hoeksema, 1991).

In summary, conclusions regarding the role of OGM in predicting depression in young people cannot be made with confidence. First, the majority of research has been crosssectional, which confines interpretation of findings to speculation about concurrent relationships. To understand OGM's role in the development of early symptoms, it is important that current, predominantly cross-sectional, research with clinical youth is complemented with community-based longitudinal studies. Specifically, associations should be tested before the onset of significant depression, and baseline depression levels need to be controlled for. In addition, differences in associations across clinical and non-clinical youth suggest that the effect of OGM may, to some degree, depend on presence of other cognitive vulnerabilities (Hamlat et al., 2015). To delineate associations between OGM and depression in community youth, therefore, research needs to control for possible moderating variables, such as rumination.

Anxiety. The association between anxiety and OGM in youth has received limited attention in this area of research. This may, in part, be due to the fact that OGM and anxiety disorders have been argued unrelated in adults (e.g., Burke \& Mathews, 1992; Wessel, Meeren, Peeters, Arntz, \& Merckelback, 2001; Williams et al., 2007). A review of the literature highlights, however, that few studies have tested this relationship and that findings are inconclusive (Zlomuzia et al., 2014). Notably, the majority of past research on OGM and anxiety was cross-sectional and analysed associations between OGM and diagnostic status (i.e. presence or absence of an anxiety disorder). Moreover, samples were relatively heterogeneous in terms of anxiety disorders investigated, which makes it difficult to disentangle possible differences in relationships between OGM and different anxiety presentations. The authors conclude that further research is needed, particularly in light of relatively robust associations between anxiety disorders and other biases in autobiographical memory (e.g., social anxiety; see Morgan, 2010, for a review).

In youth, a limited number of studies have investigated the link between OGM and anxiety, and findings were similar to those reported for adult samples. For example, in a 
longitudinal study, higher levels of OGM did not predict onset of anxiety disorders in highrisk youth (Rawal \& Rice, 2012b). Another study concluded that OGM and trait anxiety are not associated concurrently in adolescent residents of an inpatient psychiatric service (de Decker, Raes, \& Eelen, 2003). Whereas these findings may clarify associations in high-risk youth, as discussed earlier, longitudinal research with community samples is critical for a comprehensive understanding of OGM's role as a marker of vulnerability for early psychopathology. Indeed, anxiety symptoms increase steeply and predict and co-occur with depression levels during adolescence (Cummings, Caporino, \& Kendall, 2014). Moreover, depression and anxiety share common risk factors and information processing biases (Aldao, Nolen-Hoeksema, \& Schweizer, 2010; Reid, Salmon, \& Lovibond, 2006). Particularly relevant is the finding of robust associations between anxiety and the transdiagnostic risk factor of rumination, which predicts and interacts with OGM in youth to give rise to depression (Hamlat et al., 2015). Importantly, although anxiety levels increase during this time, they may not necessarily surpass diagnostic thresholds. To clarify our understanding of associations between OGM and early symptoms of anxiety, therefore, more longitudinal research is needed. Moreover, in light of high levels of comorbidity between depression and anxiety, both should routinely be included as covariates in analyses that test associations between OGM and psychopathology.

In line with the notion that OGM is problematic when it occurs alongside other risk factors, it has been suggested that OGM's ability to predict adverse psychological outcomes in youth may mirror the degree to which OGM represents underlying vulnerabilities to psychopathology (Crane et al., 2016). The predominant etiological model of OGM (the CaRFA-X model; Williams et al., 2007) supports this idea. This theoretical account identifies three cognitive vulnerabilities that are argued to have a negative impact on psychological functioning, either directly, or through independent or joint effects on OGM. Before I discuss the development of this particular model in more detail, I would like to acknowledge the existence of other OGM theories (e.g., Dalgleish et al., 2007; Valentino, 2011; Watkins \& Teasdale, 2004). As the current research focuses on the CaR-FA-X model, however, evaluation of these alternative theories is beyond the scope of this thesis and I will refer to them only where deemed necessary.

\section{Theory of Overgeneral Autobiographical Memory}

Early theoretical accounts of OGM draw on descriptions theory, which presupposes that the autobiographical memory system consists of records of events that are stored in a hierarchical system - general event memories are located at higher levels, and specific 
representations at lower levels (Burgess \& Shallice, 1996). Specifically, Williams (1996) proposed that OGM is the result of a process he termed "mnemonic interlock". Here, cue words activate self-referent categoric descriptions (at higher levels of the hierarchy), which then starts a cycle of rumination on negative self-descriptions that activates and increases accessibility of other general memories. As a result, a person retrieves autobiographical representations across the memory hierarchy, rather than moving down to specific event representations. Williams further defines OGM as a learned affect regulation strategy that develops in response to early trauma, whereby the young person avoids retrieval of specific negative memories to circumvent activation of event-related negative affect. That is, through recollection of general event memories, activation of specific event material, such as associated unpleasant affective information, is avoided. This avoidance of difficult emotional material then negatively reinforces and increases OGM. The notion of understanding OGM as a form of affect regulation was further elaborated upon in the context of a more recent extension of earlier hierarchical models of autobiographical memory, the Self-Memory System (SMS; Conway \& Pleydell-Pearce, 2000).

The Self-Memory System. In the SMS, autobiographical memory is organized hierarchically and consists of three nested levels of autobiographical knowledge ("the knowledge base"); the highest level is comprised of lifetime periods, containing knowledge of such periods' features and durations (e.g., "when I went to primary school"); the intermediate level represents general events, which are abstract recollections of repeated events (e.g., "going to the cinema"), and accounts of single events that lasted for an extended period of time (e.g., "the last school holiday"); finally, the lowest level of the hierarchy comprises concrete event-specific representations, and contains sensory and perceptual information (e.g., "my $10^{\text {th }}$ birthday party at the beach and it was sunny"). Due to the nested nature of the model, construction of specific memories involves activation of all three levels of the memory system. That is, retrieval of specific memories can occur only when activation in the knowledge base settles into a stable pattern across all three levels.

According to the SMS, people retrieve event-specific representations via two pathways. First, direct retrieval represents a relatively instantaneous, bottom-up, process, whereby a stable pattern of activation is achieved, and event-specific knowledge accessed, more or less automatically in response to environmental cues. Alternatively, specific memories can be accessed through generative retrieval. Here, a person voluntarily engages in a top-down search process through the autobiographical knowledge base, navigating from general representations at the top to event-specific representations at lower levels. This 
process is governed by executive control processes, which guide the memory search in the context of specific search criteria. Importantly, it is this form of retrieval that is believed to underlie voluntary retrieval of memories on the AMT. Similar to Williams (1996) affect regulation theory, the authors of the SMS conceptualize OGM as a result of $d y$ sfacilitation of the generative retrieval process. Specifically, it is argued that the generative phase of memory retrieval is terminated, or dysfacilitated, prior to successful retrieval of event-specific knowledge when activation of event-specific information has the potential of causing emotional distress.

The CaR-FA-X Model. Early affect regulation accounts of OGM identify trauma, or negative childhood life events, as central to the development of this phenomenon. As noted by Williams and colleagues (2007), however, OGM is also found in people with depression who do not have a history of trauma. With the goal of understanding OGM across populations with different psychopathological profiles, they subsequently elaborated on the SMS's account of OGM to propose a model $(C a R-F A-X)$ that identifies three mechanisms: Capture and Rumination, Functional Avoidance, and eXecutive control. This model is the most widely cited theory of OGM, and postulates that the three outlined components interfere with successful generative memory retrieval in various ways. The first proposed mechanism, Capture and Rumination ( $\mathrm{CaR}$ ), occurs when presentation of a cue word activates abstract self-relevant information, such as self-beliefs (e.g., happy = "I am never happy") during the retrieval search, which then captures the person's attention. Rather than moving down the SMS hierarchy and generating a specific memory, the person then ruminates on abstract information related to the self-belief, resulting in premature termination of the generative retrieval search at a general level. Repetitive ruminative thinking then further strengthens connections between abstract self-relevant information, thereby increasing likelihood of future capture errors.

Second, the Functional Avoidance (FA) mechanism, consistent with Williams' (1996) theory, refers to termination of the memory search at a general level to avoid potential elicitation of event-related negative affect. Avoidance of negative emotions positively reinforces overgeneral retrieval, which, over time, may then develop into a habitual affect regulation strategy. That is, although this style of remembering may initially be adaptive and used flexibly for negative event memories, it is believed to develop into a passive and inflexible pattern of responding to all memories with continued use (Hermans et al., 2008b). Third, executive control is argued central to successful generative memory retrieval. For example, irrelevant information must be inhibited, and retrieved information held in working 
memory. Hence, reduced e $X$ ecutive control $(X)$ can cause premature termination of the generative retrieval search at a general level. Importantly, the CaR-FA-X model emphasizes that the three mechanisms may exert effects on memory retrieval independently, or in interaction.

In adults, the majority of research on the CaR-FA-X model investigated the three mechanisms in isolation, and findings were relatively inconsistent (see Sumner, 2012, for a review). The first study to assess all components concurrently not only demonstrated that the mechanisms can predict overgeneral memory in interaction, but also that they can operate differentially across depressed and non-clinical samples (Sumner et al., 2014). These findings support conclusions drawn by previous research, namely that the relative influences of the three model components on OGM are likely to vary across people with different psychological presentations (Crane, Barnhofer, Visser, Nightingdale, \& Williams, 2007; Dalgleish, Rolfe, Golden, Dunn \& Barnard, 2008).

Research on the CaR-FA-X model in young people is limited and findings are mixed (see Hitchcock et al., 2014a; and Stewart, Hunter, \& Rhodes, 2017, for reviews). Not only do current studies provide incomplete support for this theoretical account, but evidence also suggests that the role of the mechanisms may differ in child and adolescent samples compared to research with adults. Moreover, findings across clinical and non-clinical populations vary. For example, two studies demonstrated associations between OGM and rumination and avoidance (Park et al., 2004 and Stokes et al., 2004, respectively), but the effects were limited to youth with depression or trauma-experience, and not found in control groups. Furthermore, while increased rumination and reduced executive control interacted to predict OGM in adolescents at familial risk for depression at a six-month follow-up (Rawal \& Rice, 2012a), this effect was not found at a 12-month follow-up in community adolescents (Hitchcock et al., 2014b). Available evidence raises the possibility that as for adults, the CaRFA-X model's predictive utility in youth is dependent on population being studied (clinical, or high-risk, vs. non-clinical). Indeed, the CaR-FA-X model may not function in a "one-sizefits-all” fashion (Sumner, 2012, p.4). Importantly, discrepant findings for youth and adults underscore the need for more research with young people, as these cannot be generalized across different age groups.

In summary, research on the utility of the CaR-FA-X model in young people is inconclusive. Specific limitations pertain to the predominantly cross-sectional nature of studies, the fact that the three mechanisms are typically investigated in isolation, and that interaction effects are not controlled for. Importantly, the model postulates causal 
relationships, so associations also need to be tested longitudinally. Moreover, the CaR-FA-X model proposes interactions among mechanisms, which highlights the critical importance of testing the model in its entirety so that clear conclusions about its efficacy can be drawn. Given evidence that the relative influences of the three model mechanisms differ across populations with varying psychological profiles (clinical/high-risk vs. nonclinical/community samples), analyses should also account for potential moderators such as depression levels.

Advancing our understanding of autobiographical remembering and its association with psychological well-being in this area of research need not be limited to investigation of OGM, however; examination of other qualities of memories retrieved by the AMT, such as episodic detail, may prove fruitful, which I will discuss next.

\section{Autobiographical Memory Specificity vs. Detail}

Although classification of memories in terms of specificity provides meaningful information about the event construction's degree of temporal specificity, it is important to acknowledge the multi-dimensional nature of autobiographical memories retrieved by the AMT. For example, not only can event accounts differ on the dimension of temporal specificity (i.e. whether the event occurred at a specific time and place), but also degree of detail embedded within memories. Episodic autobiographical memory detail has been defined as reflecting "reliving" of a specific past experience during recall, and includes sensory, perceptual and affective information (e.g., Addis, Wong, \& Schacter, 2008; Tulving, 2002). As outlined earlier, the SMS purports that this event-specific knowledge is accessed at the lowest level of the hierarchical autobiographical knowledge base (Conway \& PleydellPearce, 2000). As such, specific event memories differ from general event memories because they represent episodic detail relating to unique experiences. General event memories comprise more abstract and semantic autobiographical knowledge.

Considering that temporally specific memories are constructed when sensoryperceptual knowledge, or episodic detail, is activated, past research has implied that tendency to report more specific memories on the AMT indicates increased access to event-specific information (e.g., Griffith et al., 2012). Amount of reported episodic detail can vary considerably across specific event constructions, however. For example, when presented with the cue word "guilty", a young person may report "I felt guilty when I yelled at my sister" or "I felt guilty when I yelled at my sister Jane last Saturday in the evening, when she took my green shirt without asking”. Both responses are temporally specific in that they are representations of an event that, presumably, did not last longer than one day. Notably, the 
latter is a much richer elaboration of the event than the first. Individual variability may, therefore, not only exist in memory specificity on the AMT (i.e., how many specific memories young people report), but also degree of episodic detail embedded in specific memories.

Despite this possibility, only one study, with adults, has investigated the association between memory specificity and average amount of episodic detail reported in specific memories (Kyung, Yanes-Lukin, \& Roberts, 2016). The two constructs were not concurrently associated. This finding suggests that it cannot be assumed that people who retrieve more specific memories also recall more episodic detail. Moreover, Kyung et al. (2016) found that the two memory indices related differentially to adults' psychological well-being. Whereas reporting of more specific memories predicted lower concurrent levels of depression and rumination (as expected), higher average levels of detail in specific memories were associated with higher levels of depression and rumination. The authors argue that their findings may indicate that the two memory indices represent different underlying constructs.

On the one hand, Kyung et al.'s findings support the notion that adults with higher levels of depression report fewer specific memories (Sumner et al., 2010). Their findings are at odds with other research that found an association between higher levels of depressed mood and lower levels of episodic detail, however (Söderlund et al. 2014). Notably, this study recruited a clinical sample and used a different test - the Autobiographical Interviewto elicit memories. Nonetheless, considering the proposition that diminished access to episodic event memories may compromise cognitive functioning (e.g., problem-solving capacity) and increase vulnerability to psychopathology (Raes et al., 2005; Williams et al., 2007), we would expect higher levels of reported episodic detail to associate with positive psychological outcomes. Similarly, past research has argued that vivid recollections of personal experiences play an important directive role in adaptive functioning by informing and guiding a person's everyday behaviour (Pillemer, 2003). Indeed, evidence suggests that experimental induction of a more detailed memory retrieval style can improve psychological well-being and increase problem-solving in adults (Jing, Madore, \& Schacter, 2016).

Although in its early stages, the research direction pointed out by Kyung and colleagues (2016) makes an important contribution to this literature. If these two memory dimensions also represent different underlying constructs with contrasting implications for psychological well-being in youth, then clarification of their respective roles in the emergence and maintenance of psychopathology can have important theoretical and clinical implications. Rather than being protective per se, the function of greater episodic detail in 
specific memories may depend on the index of psychological functioning under investigation. Another possibility is that relationships between episodic detail and psychopathology are moderated by other cognitive vulnerabilities (e.g., rumination), as has been found for reduced specificity.

\section{Current Research}

The overarching aim of the current thesis was to shed light on longitudinal associations between OGM and psychological functioning across adolescence. In the following three studies, we investigated whether OGM predicts psychopathology; the mechanisms underlying OGM; associations between OGM and another important facet of autobiographical memory-episodic detail; and similarities and differences in how these two memory indices associate with psychological functioning. In order to achieve the most comprehensive understanding of how OGM manifests in young people, rigorous methodological and statistical strategies were used. For example, data was collected for a large sample of adolescents across multiple annual time points, and Structural Equation Modelling (SEM) used to analyse relationships. In doing so, we were able to account for the complexity in associations among multiple interrelated variables over time.

Study 1. In the initial study, we investigated the role of OGM in the development of psychopathology (depression and anxiety) in a community-based youth sample. Four gaps in the literature were addressed. First, to draw stronger conclusions about OGM's role as a predictor of early psychopathology, a sample of community adolescents was recruited and data collected across three annual assessment points. No other research has investigated these associations across more than two time points, and follow-up periods were typically shorter than one year. Second, we extended past research with community youth by including a measure of anxiety symptoms. This allowed us to test the predictive effect of OGM on both depression and anxiety, concurrently, whilst controlling for the effects these variables have on one another. Doing so is important, given that anxiety increases rapidly across the adolescent period and predicts and co-occurs with depression (Cummings et al., 2014).

Third, we controlled for the effect of rumination on OGM, depression and anxiety, and vice versa, in all analyses. Surprisingly, despite findings that rumination predicts OGM (Williams et al., 2007), and occurs alongside depression and anxiety in youth (Aldao et al., 2010), few studies have controlled for this transdiagnostic risk factor when testing longitudinal associations. Fourth, in light of the suggestion that presence of cognitive risk factors, such as rumination, may moderate associations between OGM and psychopathology in community youth (Hamlat et al., 2015), we adopted a novel analytical approach to 
delineate proposed relationships further. Specifically, when testing the effect of OGM on depression and anxiety, rather than using concurrently reported rumination levels as a moderator, we conceptualised risk as longitudinal rumination trajectories. We allocated young people to one of two groups on the basis of the pattern of rumination they reported across the three time points (stable low vs. stable elevated levels) and compared associations between OGM and psychopathology. This allowed us to not only test patterns of association across the community sample as a whole, but also as a function of rumination tendency.

Study 2. In the second study, we carried out a longitudinal test of the CaR-FA-X model in the community sample of youth recruited for Study 1. An additional fourth annual follow-up point was available and included in analyses. We addressed several gaps in the literature. First, we tested the CaR-FA-X model in its entirety, including interaction terms. Despite theoretical and empirical suggestions that the three mechanisms can exert effects on memory retrieval independently or in interaction (Sumner et al., 2014; Williams et al., 2007), only two published studies investigated interactions between rumination and various aspects of executive control (Hitchcock et al., 2014b; Rawal \& Rice, 2012a). Avoidance was not controlled for, the samples differed in terms of risk for future psychopathology, and findings were inconsistent, however. These two studies also represent the only research that investigated relationships over time. We, therefore, tested the entire model longitudinally, across four annual assessment points, in a community sample. Indeed, evaluating the CaRFA-X model in its entirety across time is crucial for understanding how OGM initially develops (Sumner, 2012).

Moreover, it is important to investigate relationships between OGM and hypothesised underlying mechanisms longitudinally whilst controlling for depression levels. Not only are all three CaR-FA-X mechanisms established covariates of depression, but different associations have been reported for depressed and nondepressed people in the past (Sumner et al., 2014). The current study, therefore, had two aims: first, to test the model across the entire sample of community youth, and second, to compare the model's applicability across groups of youth who report different patterns of early depression symptom growth (lowincreasing vs. higher-increasing levels).

Study 3. In the third study, we investigated associations between memory specificity, episodic detail and psychopathology in a subgroup of the community youth sample from Studies 1 and 2. We addressed three gaps in the literature. First, we tested concurrent associations between proportions of specific memories reported on the AMT and degree of episodic detail embedded within specific memories. Despite the common assumption that 
people who are more specific on the AMT recall more details about the past, research suggests that these two qualities of autobiographical memory are unrelated in adults (Kyung et al., 2016). No studies have investigated this research question in young people, however. Second, we examined similarities and differences in longitudinal associations between the two constructs and psychopathology (anxiety and depression). We first tested whether memory specificity and episodic detail predict psychopathology across three annual assessment points, controlling for the effect of the other variable and baseline levels of psychopathology. Only one other study has investigated these associations cross-sectionally in adults (Kyung et al., 2016).

We also tested the reverse pattern of causal influence; that is, similarities and differences in the predictive effects of the two psychopathology indices on longitudinal change in memory specificity and detail on the AMT. This has not been investigated before in young people or adults. Lastly, considering the exacerbating role of rumination in associations between memory specificity and psychopathology in community youth (e.g., Hamlat et al., 2015), moderating effects of rumination on longitudinal associations between episodic detail and psychopathology should be clarified. We, therefore, tested all longitudinal associations (memory indices predicting psychopathology, and vice versa) also as a function of rumination growth. 


\section{Chapter 2}

Study 1: Do Overgeneral Autobiographical Memories Predict Increased

Psychopathological Symptoms in Community Youth? A 3-Year Longitudinal Investigation

This chapter is composed of a published manuscript with the following citation:

Gutenbrunner, C., Salmon, K., \& Jose, P. E. (2017). Do overgeneral autobiographical memories predict increased psychopathological symptoms in community youth? A 3year longitudinal investigation. Journal of Abnormal Child Psychology. Advance online publication. doi: 10.1007/s10802-017-0278-5 


\begin{abstract}
Research suggests that an overgeneral autobiographical memory style (i.e., retrieval of general memories when instructed to retrieve a specific episodic memory) represents a vulnerability marker for depression. Although adolescence is a period of high risk for the emergence of depression, little research has investigated the associations among overgeneral memory, psychopathology, and risk factors longitudinally in a community sample in this age group. We, therefore, investigated overgeneral memory, psychopathology (depression and anxiety), and rumination (an established risk factor for psychopathology) longitudinally in 269 typically-developing youth (125 females, 144 males) across 3 annual assessment points. We sought to determine whether 1) overgeneral memory would predict psychopathology across the entire sample, and 2) whether associations would vary as a function of longitudinal rumination growth. Across the entire sample, overgeneral memory did not predict psychopathology. For youth who reported elevated, and increasing, patterns of rumination over time, transient relationships between overgeneral memory and subsequent increases in anxiety were found. We conclude that overgeneral memory may represent a vulnerability marker for adverse psychological outcomes only for youth at risk for psychopathology.
\end{abstract}




\section{Introduction}

Autobiographical remembering is central to perceptions of the self, our emotional experience, and a sense of enduring existence within a broader cultural context (Conway \& Pleydell-Pearce, 2000). For example, drawing on the lessons learned from past experiences can guide our current and future behavior and facilitate problem solving (Bluck, 2003; Nelson \& Fivush, 2004; Pillemer, 2003). Problems and biases in autobiographical remembering, conversely, are implicated in psychopathology across the lifespan (for a review see Salmon \& O'Kearney, 2014). Overgeneral memory, one such bias, is defined as the tendency to retrieve memories in a general way when asked to retrieve a specific episode of less than one day's duration (Williams \& Broadbent, 1986). For example, in response to the cue word "angry", instead of saying "when John took my book during class", an individual might say "when John takes my things" (a category of similar memories) or "when John had my book for a week" (a temporally extended memory).

Research conducted with adults shows that depressed individuals report more overgeneral memories than non-depressed individuals (Williams, 1996; Williams et al., 2007). Moreover, reporting more overgeneral memories predicts increases in depressive symptoms and a more severe course of depression (Brittlebank, Scott, Williams, \& Ferrier, 1993; Hermans et al., 2008a; Van Vreeswijk \& De Wilde, 2004; see Sumner et al., 2010; Williams et al., 2007 for reviews), suggesting that a tendency to retrieve overgeneral memories is a marker for vulnerability to depression. These and related findings provide support for the dominant theoretical model of overgeneral memory (i.e., the CaR-FA-X model; Williams et al., 2007) which proposes that higher overgenerality may occur when negative cognitive processes, such as elevated levels of rumination (CaR) and avoidant response styles (FA), and reduced availability of executive resources (X), bring the hierarchical memory retrieval search to a premature halt at the level of general rather than specific memories. The resulting diminished ability to draw on specific episodic information may then interfere with adaptive cognitive processing (e.g., problem-solving abilities) and give rise to, or exacerbate, psychopathology (Raes et al., 2005).

Adolescence is a time of particular risk for the development of depression. Longitudinal research suggests that major depression typically emerges between the ages of 11 to 14 years, with subsequent steady increases in incidence across adolescence and early adulthood, and that adolescent depression increases the risk of subsequent episodes (Cicchetti \& Toth, 1998; Thapar et al., 2012). Elucidating the precursors and mechanisms of overgeneral memory across this period can potentially shed light on the development and 
exacerbation of depression. Limited research exists on the longitudinal relationships between overgeneral memory and psychopathology across the period from late childhood into early adolescence, however. The primary aim of the current 3-year longitudinal study, therefore, was to investigate the relationships between overgeneral memory and psychopathology in a community sample ages 10 to 15 years at the first data collection point.

A number of cross-sectional studies with clinical and typically-developing children and adolescents have found a positive association between overgeneral memory and depressive symptoms (Orbach et al., 2001; Park et al., 2002; Swales, Williams, \& Wood, 2001; Vrielynck, Deplus, \& Philippot, 2007). For example, across two studies, Kuyken and Dalgleish (2011) reported that adolescents (ages 14 to 18 years) who were at greater risk of depression retrieved greater numbers of overgeneral memories than their lower-risk counterparts.

Of the few longitudinal investigations conducted with adolescents, the three that have focused on high risk youth have yielded inconsistent findings. For example, Hipwell et al. (2011) investigated the longitudinal relationships between overgeneral memory and depression for a sample of girls, half of whom screened high on a depression measure $(N=$ 195). Overgeneral memories given in response to positive, but not negative, cue words at age 11 years predicted increases in depression 1 year later. A second longitudinal study, by Rawal and Rice (2012b), followed participants between 10 and 18 years of age $(N=277)$ who were at familial risk for depression over a 12-month interval. Among adolescents reporting no depressive disorder at baseline, higher numbers of overgeneral memories in response to negative but not positive cue words predicted increases in depressive symptoms for females but not males. Higher numbers of overgeneral memories also predicted new-onset episodes for the 14 participants who developed a depressive disorder across the two time points. Similarly, Sumner et al. (2011) found that greater overgeneral memory predicted onset of a major depressive episode in older adolescents with past depression $(N=55)$, but only under conditions of concurrently reported high levels of interpersonal stress.

To draw stronger conclusions about the role of overgeneral memory in the emergence of youth psychopathology, research with at-risk samples must be complemented by longitudinal studies conducted with community youth (that is, individuals who are neither drawn from a clinical population nor specifically recruited as "at risk" for depression). A specific focus on at-risk youth increases the possibility that the patterns found for overgeneral memory might relate to heightened prior exposure to negative life events or past psychopathology (Crane et al., 2016). Therefore, while research with at-risk samples provides 
valuable insight into cognitive vulnerabilities implicated in the emergence and maintenance of psychopathology in clinical populations, there is a need for further investigation of the role of overgeneral memories in the development of new onset psychopathology. Three longitudinal studies have investigated the predictive relationships between overgeneral memory and depression in community samples. Focusing on youth ages $12-13$ years $(N=$ 174), Stange et al. (2013) found that the number of overgeneral memories (extended, categorical, and too general to be considered either extended or categorical) positively predicted depression after an 8 month follow-up period, but only when elevated overgeneral memory co-occurred with self-reported emotional abuse. Another study, by Hamlat et al. (2015), found that, for adolescents with a mean age of 12 years $(N=160)$, the number of overgeneral memories positively predicted depressive symptoms over a 9 month period, but this effect was limited to females and occurred only for individuals reporting high levels of rumination and stressful life events. In contrast, Crane et al. (2016) found neither direct nor indirect effects of overgeneral memory at age 13 years $(N=3708)$ on depressive symptoms 3 years later.

Together, these findings raise the possibility that the relationship between overgeneral memory and depression found in high-risk groups may not generalize to community adolescents; that is, overgeneral memory may not predict psychological outcomes in the absence of risk factors for psychopathology, such as elevated rumination. Within this context, some research has shown that when individuals are asked to retrieve specific one-time childhood events, some general events are invariably reported (e.g., repeated experiences, which would be classified as overgeneral memories in the current area of research). That is, reporting some general memories when asked to retrieve a specific memory seems to be normative and does not necessarily reflect poor psychological functioning (Wang \& Conway, 2004). Moreover, general memories have been found to hold as much personal meaning for young adults as unique specific memories (Peterson, Baker-Ward, \& Grovenstein, 2016). Together, these findings suggest that overgeneral memories may serve different functions, and have different consequences, in typical and atypical populations.

\section{Current Study}

By following a community sample of young people over three annual assessment points, we were able to address four gaps in the literature. First, we tested the possibility that, across the early adolescent period, reports of overgeneral memories would relate differentially to emerging psychopathology depending on whether youth reported high or low 
symptom trajectories of rumination across time. Rumination has been identified as a transdiagnostic risk factor for childhood depression and anxiety (Aldao et al., 2010; McLaughlin \& Nolen-Hoeksema, 2011). Moreover, from the theoretical perspective of the CaR-FA-X model (Williams et al., 2007), rumination contributes to the emergence and maintenance of overgeneral memory. Surprisingly, despite findings of significant associations between rumination, psychopathology, and overgeneral memory, few studies have so far investigated prospective associations between overgeneral memory and depression in youth while controlling for rumination (i.e., Hamlat et al., 2015; Rawal \& Rice, 2012b).

Second, we broadened the typical focus on the relationship between overgeneral memory and psychopathology by including anxiety in addition to depression. While Rawal and Rice (2012a) included a measure of anxiety in their study with at-risk youth, their focus was on the presence of anxiety disorders. During late childhood and early adolescence, however, it may prove fruitful to investigate associations between overgeneral memory and changes in symptoms of anxiety, rather than presence or absence of rarer anxiety disorders. Heightened anxiety is common during this developmental period, both predicting and cooccurring with depression (Cummings et al., 2014). Further, anxiety shares risk factors and information processing biases with depressed mood (Aldao et al., 2010; Reid et al., 2006).

Third, no single study has investigated associations between overgeneral memory and a range of psychopathological symptoms across more than two time points, and, with the exception of Crane et al. (2016), longitudinal studies have typically followed up participants for relatively short periods (e.g., nine months). More reliable and sensitive statistical longitudinal analyses are based on datasets with three or more data collection points per individual (Little, 2013). Fourth and last, we adopted an analytical strategy that allowed for more in-depth examination of the relationships between overgeneral memory and symptoms of anxiety and depression as a function of different patterns of change in rumination. We extend on past research that has examined rumination as a moderator of associations between overgeneral memory and psychopathology (Hamlat et al., 2015) by investigating differences in patterns of association as a function of rumination trajectories across the three time points.

We tested two hypotheses. First, we expected that, across the entire sample, increased overgenerality would not predict increases in symptoms of depression and anxiety. That is, for community youth, we expected that producing overgeneral memories would be neither indicative nor predictive of poor psychological functioning in line with previous findings (e.g., Crane et al., 2016). We considered, however, that analyses for the entire sample would 
mask differences in the relationships between overgeneral memory and psychopathology for young people reporting different levels and patterns of change in rumination across the three data collection points (Hamlat et al., 2015).

Thus, our second hypothesis was that overgeneral memory would predict increases in symptoms of depression and anxiety for participants at increased risk of poorer psychological functioning. In line with previous work, risk was operationalised as elevated longitudinal levels of rumination (Hamlat et al., 2015). Further, we expected associations between overgeneral memory and psychopathology among high ruminating adolescents for negative cues only: this latter prediction is consistent with Kuyken and Dalgleish's (2011) proposal that overgeneral memory as an affect regulation strategy may initially be limited to negative cues, but may develop into an inflexible and more globalised style of memory retrieval as psychopathology becomes more chronic. Finally, while we expected that these relationships would be evident across the first time lag, we predicted that associations would be maintained, and increase in strength, across time as the risk of psychopathology onset increases with age (the second time lag should be stronger relative to the first).

\section{Method}

\section{Participants}

Participants were recruited from eight secondary schools in urban and rural areas in New Zealand. Of the 322 participants at Time 1, 288 participated approximately 12 months later at Time 2, and 269 (144 males, 125 females) approximately 11 months later at Time 3 (for an overall retention rate of $84 \%)$. The average age of boys at Time $1(M=13.1, S D=1.2)$ was significantly greater than that of girls $(M=12.5, S D=1.0)$. Parental consent and student assent were obtained separately for each wave, giving the option of withdrawal at follow-up stages. Attrition at follow-up was primarily due to the student being absent on the day of testing or having moved to an unknown school. The majority $(80.1 \%)$ of participants identified as New Zealander or European New Zealander, 5.3\% as Mãori, 7.9\% as Asian, 3.4\% as Pacific Islanders, and the remaining 3.4\% classified their ethnicity as Other. Across participating schools, children from low to high socio-economic bands were represented in the sample, with the majority of children from the middle socio-economic band. This research was approved by the School of Psychology Human Ethics Committee, under delegated authority to the Victoria University of Wellington's Ethics Committee. 


\section{Measures}

Depression. Participants completed the 12-item Children's Depression Inventory-2 Self-Report Short Version (CDI-2:SR[S]; Kovacs, 1985, 1992). Participants were presented with three sentences per item (e.g., "I hate myself", "I do not like myself", "I like myself"), and asked to select the one response option that best corresponds to how they had been feeling over the past 2 weeks. All items are scored on a 3 -point scale $(0=$ no symptoms, $1=$ mild symptoms, 2 = severe symptoms). The measure demonstrated good internal consistency across the three waves of data collection $(\alpha \mathrm{s}=.74$ to .83$)$.

Rumination. An abbreviated version of the Ruminative Response subscale of the Children's Response Styles Questionnaire (CRSQ; Abela, Brozina, \& Haigh, 2002) was administered to assess rumination, defined as "comparison of one's current situation with some unachieved standard" (Treynor, Gonzalez, \& Nolen-Hoeksema, 2003, p. 256). Participants were presented with statements about cognitive processing styles (e.g., "When I am sad, I think about how angry I am with myself') and asked to indicate how often they engage in this response style on a 4 -point scale $(1=$ almost never $; 2=$ sometimes; $3=$ often; 4 $=$ almost always). The 6-item scale yielded good internal reliability across the three waves of data collection $(\alpha \mathrm{s}=.77$ to .85$)$.

Anxiety. The Revised Children's Manifest Anxiety Scale-2 (RCMAS-2; Reynolds \& Richmond, 2008) Short Form is a 10-item self-report measure of general anxiety. Items tap into three facets of anxiety: social concerns (e.g., "I feel someone will tell me I do things the wrong way”), physiological anxiety (e.g., "Often I feel sick in my stomach”), and worry (e.g., "I often worry about something bad happening to me"). Participants respond with either YES or NO. The RCMAS-2 Short Form yields a total anxiety score, and demonstrates good construct validity (e.g., Lowe, 2015). Good internal reliability was obtained across the three waves of data collection $(\alpha \mathrm{s}=.75$ to .80$)$.

Autobiographical memory. An adapted written version of the Minimal Instructions Autobiographical Memory Test (Mi-AMT; Debeer et al., 2009) was used to assess specificity and generality of autobiographical memory. Prior research has found this version of the AMT, which does not include example or practice items, to be more sensitive to overgeneral autobiographical memory in non-clinical populations than Williams and Broadbent's (1986) original measure (Debeer et al., 2009). On the basis of a pilot test, we made slight alterations to the instructions provided by the Mi-AMT, as we found that our participants, who were younger than in Debeer's research, required additional information regarding type and specificity of memories sought. Participants were presented with 10 cue words in a fixed 
order, alternating between positive (happy, lucky, proud, excited, relaxed) and negative valence (sad, angry, lonely, guilty, scared). The cues were adopted from past research with participants of comparable age (Hipwell et al., 2011; Raes et al., 2010). Students were told that they were completing a memory test, and were asked to think of and write down a specific event or situation of which each cue word reminded them. The current test differed from the Mi-AMT in that we gave an example of a correct and an incorrect response to a cue word not included in the test word list, but instructions regarding the degree of desired specificity of memories were minimal. In line with the Mi-AMT, and unlike the original AMT, no practice items were provided. Students were given 1 minute to write down their memory for each cue word. Participants provided their responses in writing; our previous research (Glynn, Salmon \& Jose, 2016) has shown that reporting mode (writing vs speaking) does not significantly influence the number of specific and overgeneral memories children provide on the AMT.

Memory coding and reliability. Memories were assigned to one of eight different memory categories. In line with pre-established AMT coding guidelines (Griffith et al., 2012; Williams et al., 2007), a memory referring to a single specific event lasting less than 1 day was coded as specific (e.g., "my last birthday party"); a memory referring to an event that lasted longer than 1 day was coded as extended (e.g., "visiting Spain last summer"); a memory referring to a collection of similar events repeated over time was coded as categoric (e.g., "playing with my friends"); and a verbal association to the cue (e.g., "movie" in response to cue word happy) was coded as a semantic associate. Events that had not yet happened were coded as future oriented (e.g., "going swimming next Sunday"); repetitions of previously reported memories as repeated; incomplete responses (providing partial information about an event) as incomplete; and failure to provide a response as an omission.

One of the complexities in current research is inconsistency in conceptualization of overgenerality. For example, whereas Hamlat et al. (2015) analyzed total number of categoric and extended memories, Crane et al. (2016) analyzed specific memories, with all other types of responses, including omissions and semantic associates, being considered non-specific. In order to obtain the fairest picture of associations between study variables, we integrated past research and adopted a range of conceptualizations. Six different indices of overgeneral memory - as utilized in previous research — were tested separately as part of all analyses. First, proportions of specific and overgeneral memories (extended and categoric), and, due to inconsistencies in findings of associations between psychopathology and overgeneral memories provided in response to cues of different emotional valence (e.g., Hipwell et al., 
2011; Kuyken \& Dalgleish, 2011), proportions of specific and overgeneral memories when split by cue word valence (positive or negative). All proportions were computed using total number of responses to cue words excluding omissions (Griffith et al., 2012).

To establish reliability, all memories (100\%) were coded independently by two raters. Average Cohen's kappa across the three waves of data, totalling 8,070 memories, was .77. A dichotomous memory specificity variable was also computed, with specific memories being assigned a value of 1 , and remaining non-specific memories being coded as 0 . Average kappa was .85 across the three waves, suggesting high interrater agreement with respect to whether a memory was considered specific or not.

\section{Procedure}

Eight out of 18 schools initially contacted about the study agreed to participate (44\%). Parent consent forms were distributed by the school to appropriate-age classes; the percentage of forms returned from the number distributed is unknown. Across all waves, participants were assessed at school, in groups of approximately 10 students during class time. At the beginning of each session, participants were told their answers would be confidential, and that responses would be traced back to participants and schools contacted only if responses raised concerns about their wellbeing. On completion of assent forms, the first part of assessment entailed administration of the adapted Mi-AMT. The second part of the session involved completion of self-report questionnaires. Participants were then debriefed about the purpose of the study and invited to ask questions. Sessions, on average, lasted 45 minutes. Data collection and coding were carried out by trained postgraduate students and research fellows.

\section{Missing Data}

Analysis of patterns of item-level missing data in self-report measures using Little's MCAR test (Little \& Rubin, 2002) showed that data were missing completely at random, Little's MCAR $\chi^{2}(2716, N=269)=2711.78, p=.520$. Across the three data collection waves, total percentage of missing items was $1.90 \%$. Using SPSS, an expectation-maximization (EM) algorithm was employed to impute missing values, on the basis of which scale totals were subsequently computed und used for analyses.

\section{Analytic Strategy}

Following preliminary analyses to obtain descriptive statistics, autoregressive crosslagged models were estimated to test for prospective associations among study variables. Six 
models adopting different indices of overgeneral memory, as outlined earlier, were tested separately using the structural equation modelling software package AMOS Version 23 (Arbuckle, 2014). Due to the large number of multiple comparisons carried out, a Šidák correction for multiple comparisons was computed and applied to all analyses, resulting in rejection of the null hypothesis only if the $p$-value was equal to or smaller than .015 (Šidák, 1967). Bias-corrected (BC) 98.5\% confidence intervals (CI) were bootstrapped (2000 samples) for all prospective association analyses. The true population value for a given estimate lies within the upper and lower limits of this interval.

First, baseline models with freely varying autoregressive coefficients (associations between scores on a single measure across time points) were compared to models where such coefficients were constrained to equality across the three waves. Because we expected to find associations of different magnitude across the two time lags on theoretical grounds (Time 1 to Time 2, and Time 2 to Time 3), cross-lagged pathways were allowed to vary freely in all models. Constraining them would entail averaging strengths of associations, and this approach can conceal time-specific effects. Chi-square difference tests were carried out to identify the most parsimonious cross-lagged autoregressive models including measures of depression, anxiety, and rumination, and one of the six indices of overgeneral memory at a time. Variables were allowed to covary with one another at each time point. This process resulted in a final selection of six best-fitting models. Gender and age were included as covariates in all analyses. To test our first hypothesis, the final models' path estimates were assessed for significant prospective associations between overgeneral memory and psychopathology.

Next, a latent class growth analysis (LCGA) was carried out to identify subgroups of individuals in the sample with different growth trajectories on the rumination measure across the three data collection waves. While the traditional latent growth curve modeling approach identifies an overall trajectory of change for the population and estimates individual deviation from this curve, LCGA assumes that the population of interest is comprised of distinct subgroups with varying patterns of temporal change on a given measure (Muthén \& Muthén, 2000). The best-fitting model with the optimal number of subgroups is considered to have the smallest Bayesian Information Criterion (BIC) and a significant Lo, Mendell, and Rubin's (2001) likelihood ratio test statistic (LMR-LRT) when comparing a current model with $k$ groups to a model with k-1 groups (e.g., Jung \& Wickrama, 2008; Muthén \& Muthén, 2000). The software package Mplus Version 7 (Muthén \& Muthén, 1998-2015) was used for analyses. 
To test our second hypothesis, omnibus tests of interaction effects between rumination trajectory group membership and overgeneral memory indices in predicting anxiety and depression were first carried out. If an interaction effect was significant across either time lag, stratified, multi-group analyses were then carried out to determine differences in prospective associations across subgroups.

\section{Results}

\section{Attrition}

Analyses were conducted to determine whether a participant's gender, age, symptoms of depression, anxiety, and rumination, and proportions of specific and overgeneral memories at Time 1 were systematically related to whether or not they participated in all three data collection waves. A Pearson's chi-square test showed no significant relationship between gender and attrition group membership (completers vs. non-completers), $\chi^{2}(1, N=322)=$ $0.81, p=.369$. A multivariate analysis of variance was carried out with attrition status as the independent variable and rumination, anxiety, depression, and overgenerality as the dependent variables. A non-significant overall multivariate group effect for attrition was found; $F(4,317)=1.32, p=.263$, Wilk's $\Lambda=0.984, \eta_{\mathrm{p}}^{2}=.02$. Despite a non-significant multivariate main effect, univariate between-participants effects suggested that participants who completed all three waves of data collection displayed slightly lower levels of rumination at Time $1(M=1.94, S D=0.65)$ than non-completers $(M=2.15, S D=0.69), F(1$, $320)=4.43, p=.036, \eta_{\mathrm{p}}^{2}=.02$. Possible implications of this difference are addressed in the limitations section of the Discussion.

\section{Descriptive Statistics}

Table 1 reports correlations among study variables across the three waves of data collection. In light of the predictions, several zero order correlations were notable, albeit small in size. With regard to correlations involving the memory indices, higher proportions of specific memories at Time 2 were significantly correlated with lower depression scores at Time 1 and Time 2, and higher proportions of specific memories at Time 3 were correlated with lower depression scores at Time 2. Higher proportions of specific memories at Time 2 were correlated with higher anxiety scores at Time 1 and 2 . In contrast, proportions of overgeneral memories did not correlate significantly with depression or anxiety at any point in time. Depressive and anxious symptom constructs displayed good stability across data collection points, and these measures inter-correlated significantly in the expected direction. 
Table 2 presents study variables' means and standard deviations. Mean levels of overgeneral memory and specificity are consistent with those reported in past research (Hipwell et al., 2011; Rawal \& Rice, 2012b). 
Table 1

Correlations Between Study Variables $(N=269)$

\begin{tabular}{|c|c|c|c|c|c|c|c|c|c|c|c|c|c|c|c|}
\hline & Measure & 1 & 2 & 3 & 4 & 5 & 6 & 7 & 8 & 9 & 10 & 11 & 12 & 13 & 14 \\
\hline 1. & Prop. OGM T1 & - & & & & & & & & & & & & & \\
\hline 2. & Prop. OGM T2 & $.33 * *$ & - & & & & & & & & & & & & \\
\hline 3. & Prop. OGM T3 & $.18 * *$ & $.28^{* *}$ & - & & & & & & & & & & & \\
\hline 4. & Prop. SPM T1 & $-.82 * *$ & $-.31 * *$ & $-.24 * *$ & - & & & & & & & & & & \\
\hline 5. & Prop. SPM T2 & $-.29 * *$ & $-.76^{* *}$ & $-.37 * *$ & $.36^{* *}$ & - & & & & & & & & & \\
\hline 6. & Prop. SPM T3 & $-.20 * *$ & $-.26 * *$ & $-.85 * *$ & $.27 * *$ & $.38^{* *}$ & - & & & & & & & & \\
\hline 7. & CDI-2 T1 & -.04 & .07 & .04 & .01 & $-.18 * *$ & -.07 & - & & & & & & & \\
\hline 8. & CDI-2 T2 & .01 & .10 & .07 & -.02 & $-.20 * *$ & $-.13^{*}$ & $.55^{* *}$ & - & & & & & & \\
\hline 9. & CDI-2 T3 & .04 & .08 & .00 & -.02 & -.12 & -.04 & $.53 * *$ & $.62^{* *}$ & - & & & & & \\
\hline 10. & RCMAS-2 T1 & -.07 & .10 & .11 & -.04 & $-.21 * *$ & -.09 & $.61 * *$ & $.41^{* *}$ & $.38^{* *}$ & - & & & & \\
\hline 11. & RCMAS-2 T2 & .01 & .06 & .09 & -.06 & $-.14^{*}$ & -.10 & $.48 * *$ & $.65^{* *}$ & $.47^{* *}$ & $.61 * *$ & - & & & \\
\hline 12. & RCMAS-2 T3 & -.05 & .05 & .05 & .03 & -.08 & -.04 & $.39 * *$ & $.46^{* *}$ & $.61^{* *}$ & $.50^{* *}$ & $.58 * *$ & - & & \\
\hline 13. & CRSQ T1 & .03 & .05 & .01 & -.11 & $-.14 *$ & -.04 & $.50 * *$ & $.35^{* *}$ & $.30^{* *}$ & $.55^{* *}$ & $.47 * *$ & $.36^{* *}$ & - & \\
\hline 14. & CRSQ T2 & .05 & .01 & .04 & -.05 & $-.13 *$ & -.09 & $.33 * *$ & $.53^{* *}$ & $.35^{* *}$ & $.37 * *$ & $.58 * *$ & $.39^{* *}$ & $.63^{* *}$ & - \\
\hline 15. & CRSQ T3 & .04 & .07 & .04 & -.06 & $-.15^{*}$ & -.08 & $.33 * *$ & $.45^{* *}$ & $.55^{* *}$ & $.41^{* *}$ & $.45^{* *}$ & $.61^{* *}$ & $.50 * *$ & $.66^{* *}$ \\
\hline
\end{tabular}

Note . T1 = Time 1; T2 = Time 2; T3 = Time 3; Prop. OGM = Proportion of overgeneral autobiographical memories (sum of categoric and extended memories); Prop. SPM = Proportion of specific autobiographical memories; CDI-2 = Children's Depression Inventory-2; RCMAS-2 = Revised Children's Manifest Anxiety Scale-2; CRSQ = Children's Response Styles Questionnaire.

$* * p<.01 ; * p<.05$. 
Table 2

Means and Standard Deviations

\begin{tabular}{|c|c|c|}
\hline Measure & $M$ & $S D$ \\
\hline$\overline{\text { Prop. OGM T1 }}$ & .23 & .20 \\
\hline Prop. OGM T2 & .19 & .18 \\
\hline Prop. OGM T3 & .19 & .17 \\
\hline Prop. SPM T1 & .65 & .24 \\
\hline Prop. SPM T2 & .68 & .22 \\
\hline Prop. SPM T3 & .71 & .20 \\
\hline CDI-2 T1 & 4.30 & 3.12 \\
\hline CDI-2 T2 & 4.57 & 3.60 \\
\hline CDI-2 T3 & 5.52 & 3.87 \\
\hline RCMAS-2 T1 & 2.95 & 2.50 \\
\hline RCMAS-2 T2 & 3.17 & 2.60 \\
\hline RCMAS-2 T3 & 3.74 & 2.77 \\
\hline CRSQ T1 & 1.94 & 0.65 \\
\hline CRSQ T2 & 2.02 & 0.76 \\
\hline CRSQ T3 & 2.07 & 0.70 \\
\hline \multicolumn{3}{|c|}{$\begin{array}{l}\text { Note. T1 = Time 1; T2 = Time 2; T3 = Time 3; } \\
\text { Prop. OGM = Proportion of overgeneral } \\
\text { autobiographical memories (sum of categoric } \\
\text { and extended memories); Prop. SPM = } \\
\text { Proportion of specific autobiographical } \\
\text { memories; CDI-2 = Children's Depression } \\
\text { Inventory-2; RCMAS-2 = Revised Children's } \\
\text { Manifest Anxiety Scale; CRSQ = Children's } \\
\text { Response Styles Questionnaire. }\end{array}$} \\
\hline
\end{tabular}

\section{Model Fitting}

Step-wise relaxation of autoregressive parameter constraints did not provide a significantly better fit to the data for any of the six models, regardless of which overgeneral memory index was adopted. Consequently, the most parsimonious models contained autoregressive pathways that were constrained across the two time lags. The Comparative Fit Index (CFI) and Root Mean Square Error of Estimation (RMSEA) were computed to assess model fit. Cross-lagged pathways were allowed to vary freely on theoretical grounds, as discussed earlier. The final six models exhibited acceptable fit to the data, with $\chi^{2}(31, N=$ 269) values ranging from 67.92 to $79.51, p s<.001, C F I \mathrm{~s}=.97$ to .98 , and RMSEAs $=.07$. 


\section{Prospective Associations between Overgeneral Memory and Psychopathology for the Entire Sample}

To test our hypotheses, cross-lagged path estimates were first examined across the entire sample, and then as a function of trajectory subgroup. Across the entire sample, none of the six overgeneral memory indices predicted change in symptoms of anxiety or depression across either time lag. This result is consistent with our first hypothesis, namely that the association between an overgeneral memory retrieval style and psychopathology for the entire community sample would be weak to non-existent.

\section{Identification of Latent Classes}

The initial test of prospective relationships among overgeneral memory and psychopathology was performed on the entire sample, but follow-up moderation analyses were conducted using classes based on rumination symptom trajectories. In order to perform the latter analyses, latent class growth modelling analyses were carried out to identify subgroups of adolescents with varying developmental trajectories on the rumination measures across the three data collection waves. On the basis of the LMR-LRT test, a three-group solution was found to be optimal. Testing models with more subgroups resulted in nonsignificant LMR-LRT test statistics and only marginal reductions in BIC values, suggesting that additional classes did not clarify the composition of the model. Intercepts and slopes are reported. The three subgroups were: 1) a group displaying stable low levels of rumination symptoms over time ( $N=161, I=1.63, S=-.01, p<.001$ and $p=.686$, respectively), 2$)$ a group displaying slightly higher and increasing symptoms $(N=83, I=2.18, S=.21, p<.001$ and $p=.002$, respectively), and 3) a group displaying stable high levels of rumination symptoms $(N=25, I=3.20, S=.04, p<.001$ and $p=.725$, respectively).

\section{Comparison of Prospective Associations across Rumination Trajectory Subgroups}

As the small size of the high rumination trajectory subgroup $(N=25)$ renders estimation of parameters among 12 variables statistically untenable, medium and high growth subgroups were collapsed into a single group, resulting in low vs. medium/high group comparisons. First, differences in levels of overgeneral and specific memory across the low and medium/high rumination trajectory subgroups were assessed using repeated measures ANOVA. Age and gender were included as covariates in all analyses. Findings suggested absence of trajectory subgroup main effects for overgenerality and specificity variables, respectively; $F \mathrm{~s}(1,265)=0.70$ and 3.55, $p \mathrm{~s}=.404$ and .061 . Further, the Levene's Test for equality of variances was non-significant for proportions of overgeneral memories, 
suggesting that the variance of proportions of overgeneral memories reported did not differ across the two subgroups. There was, however, a significant difference in variance of proportions of specific memories reported across the two rumination subgroups at Time 2 , with greater variance in the medium/high rumination group, $F(1,267)=4.43, p=.036$.

Next, omnibus tests, using one of the six overgeneral memory indices at a time, were run to test whether rumination trajectory group membership and overgeneral memory interacted to predict anxiety and depression. Cross-lagged effects of anxiety and depression on each other were controlled for. One significant interaction was found: proportions of overgeneral memories to negative cues and rumination trajectory group membership in interaction at Time 1 predicted anxiety at Time $2, \beta=.25, p=.003$, BC 98.5\% CI [.06, .45]. Follow-up multi-group analyses showed that in the medium/high rumination subgroup, but not the low rumination subgroup, the proportion of overgeneral memories to negative cue words at Time 1 predicted an increase in levels of anxiety at Time $2, \beta=.25, p=.006, \mathrm{BC}$ $98.5 \% \mathrm{CI}[.04, .43]$. In the low rumination subgroup, the association between the proportion of overgeneral memories to negative cue words at Time 1 and anxiety at Time 2 approached significance; higher overgeneral memory proportions were associated with decreases in anxiety, $\beta=-.13, p=.016$, BC $98.5 \%$ CI $[-.27, .003]$.

\section{Results Summary}

In summary, the findings provided support for our first hypothesis, namely that elevated overgeneral memory would not predict increases in depression or anxiety across time for the entire community sample. We obtained partial support for our second hypothesis that associations between overgeneral memory and psychopathology would vary as a function of rumination tendencies, and that associations would be evident for overgeneral memory to negative, but not positive, cue words. Only in youth with elevated and increasing rumination levels across time was transient evidence of a predictive association between overgeneral memories to negative cue words and subsequent increases in anxiety symptoms found. Increases in overgeneral memory did not predict increases in psychopathology across the low trajectory subgroup. Unexpectedly, overgeneral memory to negative cue words at Time 1 modestly predicted reductions in anxiety symptoms at Time 2 in this group.

\section{Discussion}

The aim of the current study was to investigate longitudinal associations among overgeneral autobiographical memory, rumination, and psychopathology in community youth. Consistent with our first hypothesis, across the entire sample, proportions of overgeneral 
memories did not predict increases or decreases in symptoms of psychopathology (anxiety or depression) over time. Partially consistent with our second hypothesis, however, for the subgroup of youth who reported higher trajectories of rumination across the three waves, proportions of overgeneral memories generated in response to negative cue words at Time 1 predicted an increase in anxiety (but not depression) at Time 2. This pattern was relatively weak and was not replicated from Time 2 to Time 3. For the subgroup reporting low rumination trajectories, proportions of overgeneral memories reported did not predict increases in psychopathology. Together, these findings suggest that the role of overgeneral memory as a vulnerability marker for adverse outcomes may be limited to youth who are at elevated risk of psychopathology, as indexed by reports of heightened levels of rumination across an extended period of time.

The only evidence obtained for a link between overgeneral memory and psychopathology was for the outcome of anxiety. Research on overgeneral memory in youth has typically focused on relationships with depression and/or trauma (for a review, see Hitchcock et al., 2014a), and anxiety has received very limited research attention. Although Rawal and Rice (2012b) assessed predictive associations between overgeneral memory and new-onset anxiety disorders, subclinical anxiety symptoms were not included in their analyses. Moreover, Hamlat et al. (2015) reported an association between overgeneral memory and psychopathology in youth with heightened levels of rumination, but did not assess relationships with anxiety. Yet anxiety occurs very commonly during childhood and adolescence and is an established precursor of later psychopathology, including depression (Cummings et al., 2014; Moffitt et al., 2007). Further, anxious individuals manifest negative repetitive thinking to a similar degree as individuals experiencing depression (McEvoy, Watson, Watkins, \& Nathan, 2013). In summary, rumination may exacerbate the influence of overgeneral memories on the development of anxiety across early adolescence. It may be, therefore, that overgeneral memory plays multiple roles in the development and maintenance of psychopathology, and that associations vary depending on the levels and nature of concurrent cognitive vulnerabilities.

Our finding that, for our higher rumination subgroup, overgeneral memory predicted increases in anxiety but not increases in depression may reflect differences in covariates between our and other studies. Our path model included the variables anxiety and depression, controlling for the effect of each variable on the other, and accounted for rumination tendencies across time. This statistical approach is important because adolescents at risk of depression engage in high levels of rumination (Kuyken, Watkins, Holden, \& Cook, 2006) 
and are also likely to experience heightened anxiety (Pine, Cohen, Gurley, Brook, \& Ma, 1998). Unlike previous research, which has tended to focus on depression to the exclusion of anxiety, this approach enabled us to isolate the unique relationships between overgeneral memory and psychopathology while controlling for known covariates and for baseline levels of outcome variables.

In contrast to patterns of findings for individuals who engaged in higher levels of rumination, for the subgroup reporting stable lower levels of rumination, neither proportions of overgeneral nor specific memories were significantly related to increases in symptoms of anxiety or depression. These findings are consistent with those reported by Crane et al. (2016); despite adopting a different methodology from our own to elicit memories, these researchers found no significant longitudinal associations between overgeneral memory and depression across two time points in a large community adolescent sample. Collectively, therefore, these findings suggest that community samples do not yield significant relationships between memory overgenerality (whether conceptualized as higher overgenerality or low specificity) and psychopathology.

One might argue that generation of overgeneral memories is not indicative of risk within community samples. Research investigating diverse research questions relating to autobiographical memory in non-clinical samples finds that, despite being asked to retrieve specific memories, invariably some participants recall general memories (e.g., Wang \& Conway, 2004). Memories of repeated experiences are comparable to specific memories when rated by participants for their biographical importance and personal significance (e.g., Peterson et al., 2016), and, when used to develop relationships with others, relate to psychological well-being to a similar extent as unique memories (Waters, 2014). Despite a number of differences amongst these various studies in mode of memory retrieval and task instructions, the findings, in combination with our own, suggest that retrieval of non-specific memories in non-clinical samples, even when asked to retrieve specific experiences, is not necessarily a harbinger of poor psychological outcomes.

Particularly interesting was our finding that the mean proportions and variances in overgeneral memories did not significantly differ between our lower and higher rumination subgroups despite their different relationships with psychopathology. Our data suggest that the function and implications of overgeneral memories vary according to the level of rumination experienced by the young person. For the low rumination group, the prospective association between overgeneral memories to negative cue words and reductions in anxiety approached significance. This finding is consistent with theory and research suggesting that 
retrieval of overgeneral memories can help an individual avoid the emotions aroused by specific distressing memories (Ganly, Salmon, \& McDowall, 2016; Hermans et al., 2008b; Raes et al., 2003; Williams et al., 2007). Over time, however, this pattern, if repeated, may become inflexible and counterproductive (Hermans et al., 2008b). Individuals engaging in higher levels of rumination may be particularly vulnerable to this kind of emotion regulation strategy, as rumination is also associated with avoidant behaviors and cognitions (Lyubomirsky, Layous, Chancellor, \& Nelson, 2015; Moulds, Kandris, Starr, \& Wong, 2007). For the higher ruminators, therefore, recalling overgeneral memories may contribute to a cycle of attempted avoidance, which maintains and exacerbates poor psychological functioning over time (Hermans et al., 2008b). Consistent with this notion are findings by Raes et al. (2005) that overgeneral memory mediated the association between rumination and impaired problem solving in depressed women.

The positive link between overgeneral memory and anxiety for high ruminators was found only across the first time lag. This finding is inconsistent with our expectation that associations would emerge early and be perpetuated as risk for psychopathology increases. One possible explanation may be that time-varying factors not measured in our study interacted with rumination and overgeneral memory to predict anxiety. For example, recent findings have identified stress as central to the association between overgeneral memory and psychopathology in youth (Hamlat et al., 2015; Stange et al., 2013). Hamlat et al. (2015) found that stress and rumination in interaction moderated the association between overgeneral memory and depression in a community youth sample. As we did not assess stress, we could not test this hypothesis.

Consistent with our prediction, significant relationships between overgeneral memory and anxiety were found for participants' responses to negative rather than positive cues. Although research provides evidence for a uni-dimensional AMT factor structure (Griffith et al., 2009; Heron et al., 2012), mixed findings regarding cue valence effects have been reported in past studies with youth (e.g., Hipwell et al., 2011; Kuyken \& Dalgleish, 2011; Rawal \& Rice, 2012b; see Heron et al., 2012). Kuyken and Dalgleish (2011) theorise that, in adults, valence effects may be less common because they have developed a global avoidance style relating to both positive and negative autobiographical memories. In young adolescents who report lower levels of psychopathology, as in the current sample, avoidant responding may still be restricted to negative memories. As rumination leads to depression over time, overgenerality to both negative and positive cues may become evident. 
How might these findings advance theory and clinical practice? From a theoretical perspective, our findings that overgeneral memory predicted increases in anxiety only for the medium/high rumination group is broadly consistent with the CaR-FA-X model, which proposes that rumination and overgeneral memory may interact over time to contribute to and maintain psychopathology (Williams et al., 2007). Our findings highlight: 1) the importance of anxiety as an outcome in a sample of young people, and 2) that overgeneral memory retrieval per se may not be indicative of poor psychological outcomes in community youth but rather represent a marker for adverse outcomes in interaction with other maladaptive cognitive vulnerabilities. Particularly relevant for clinical contexts, the current findings identify rumination as critical to understanding associations between overgeneral memory and psychopathology in youth (see also Hamlat et al., 2015). Indeed, our findings support past research findings demonstrating that rumination exacerbates the effects of other cognitive vulnerabilities on psychopathology (Ciesla \& Roberts, 2007). Collectively, these findings underscore the importance of targeting rumination in clinical interventions with youth as with adults (e.g., Nolen-Hoeksema \& Watkins, 2011).

In addition to the lack of a measure of stress, a limitation of our study is that students who only completed the first data collection wave reported significantly higher levels of rumination at Time 1 than youth who remained in the study for all three time points. Although it raises the issue of lack of generalizability, this difference yielded a very small effect size, and the current study's low drop-out rate and large sample size buffer against possible effects of such a bias. Further, although gender was included as a covariate, we did not analyze potential gender differences. Including gender as an additional multi-group factor in models would have substantially increased the Šidák significance threshold adjustment, compromising chances of detecting significant differences in associations of primary interest. Finally, our sample was relatively homogenous with respect to ethnicity; given the impact of cultural factors on autobiographical memory, this fact also limits the generalizability of our findings (Hipwell et al., 2011; Wang \& Conway, 2004).

In conclusion, our findings suggest that overgeneral memory plays a role, albeit a relatively weak one, in fostering poor psychological functioning in community youth who have, by early adolescence, established relatively elevated and increasing levels of the psychopathology risk factor of rumination. In contrast, well-functioning youth may opt for an overgeneral memory retrieval style from time to time to regulate and cope with occasional difficult emotional experiences without adverse consequences. 


\section{Chapter 3}

\section{Study 2: What Predicts Overgeneral Autobiographical Memory in Youth? Testing the}

\section{CaR-FA-X Model Longitudinally in Adolescents}

The previous chapter of this thesis examined whether OGM predicts psychopathology in a large community sample across three annual assessment points. The current chapter builds on Study 1 by investigating possible underlying mechanisms of OGM itself. Specifically, in Study 2, we tested the predominant model of OGM - the CaR-FA-X model (Williams, 2006; Williams et al., 2007) — in the same sample of youth across four annual time points. As discussed in Chapter 1, research on the applicability of this particular theoretical account of OGM in adolescence is lacking (Stewart et al., 2017). Moreover, existing research, for the most part, tested the cross-sectional effects of the three model components on OGM in isolation. Arguably, longitudinal investigation of the model as a whole, including interaction terms, is needed to determine if, and how, these mechanisms influence OGM (Sumner, 2012). In light of the notion that OGM may represent a psychological vulnerability, it is particularly important to investigate this research question in adolescence, a time when psychopathological symptoms increase steeply (Thapar et al., 2012). Identification of cognitive risk factors that may render a young person more susceptible to developing an OGM tendency may have important theoretical and clinical implications. Ultimately, targeting such vulnerabilities as part of intervention programmes may help prevent increases in both OGM and early psychopathology.

This chapter is composed of a manuscript that is currently under second review with Development and Psychopathology:

Gutenbrunner, C., Salmon, K., \& Jose, P. E. (2017). What predicts overgeneral memory in youth? Testing the CaR-FA-X model longitudinally in community adolescents. Manuscript under review. 


\begin{abstract}
Overgeneral autobiographical memory (OGM) - the tendency to report general memories when asked to report specific event recollections - has been implicated in the development and maintenance of psychopathology. The dominant model of OGM, the CaR-FA-X model (Williams et al., 2007), proposes that three cognitive processes (increased rumination and avoidance, and reduced executive control) either independently, or in interaction, interfere with successful memory retrieval. Although psychopathology increases significantly during adolescence, no research has tested this model in its entirety, including interaction effects, longitudinally in community youth. Therefore, we tested the model with 323 adolescents (152 females, 171 males) across four annual assessment points. Increased avoidance predicted higher OGM from Time 3 to Time 4, but this association was stronger for youth with elevated depressive symptoms across the 4 waves, and limited to memories generated in response to negative cue words. This finding may indicate that youth with stable higher levels of depression remember in an overgeneral way to avoid re-elicitation of negative event-related emotions. In youth with lower depression levels across time, the CaR-FA-X mechanisms did not predict OGM.
\end{abstract}




\section{Introduction}

Disruptions in autobiographical memory, our memory for our personal past, play a key role in the development and maintenance of psychopathology (e.g., Dalgleish \& WernerSeidler, 2014; Salmon \& O'Kearney, 2014). One such disruption is overgeneral memory $(\mathrm{OGM})$, which is the tendency to recall general memories when asked to retrieve a specific autobiographical episode (Williams et al., 2007). For example, in response to the cue word "angry", a specific autobiographical memory would be "last Monday when I fought with my sister". In contrast, "when I fight with my sister", summarizing a category of experiences, is an overgeneral memory. In youth and adults, recalling greater numbers of overgeneral memories has been found to predict increased symptoms and future episodes of psychopathology, particularly depression (for reviews, see Hitchcock et al., 2014a; Sumner et al., 2010; Williams et al., 2007). Further, in combination with heightened rumination, OGM predicts increases in adolescent anxiety over time (Gutenbrunner, Salmon, \& Jose, 2017). One way in which reduced specificity may contribute to the development of psychopathology is through interfering with effective problem-solving (Jing et al., 2016). Understanding the factors that contribute to the development and maintenance of OGM in youth can therefore shed light on the development of psychopathology.

The dominant model of OGM, the CaR-FA-X model (Williams et al., 2007, see Figure 1), proposes that three mechanisms, either separately or in interaction, cause and maintain OGM, and thereby lead to and maintain psychological difficulties: Capture and Rumination (CaR), Functional Avoidance (FA), and Executive Control (X). To date, no research has tested this model in its entirety, adopting a longitudinal design incorporating all three factors and including interaction effects in a sample of adolescent youth. Longitudinal investigation of OGM in adolescence is particularly important given the dramatic increase in onset of both anxiety and depression during this period (Cicchetti \& Toth, 1998; Costello et al., 2006). In light of research that highlights OGM as a vulnerability marker of psychopathology, it is crucial to investigate mechanisms underlying this risk factor prior to the onset of significant psychopathology. Adolescence is a time when psychopathology such as depression typically first emerges (Kessler et al., 2007); clarification of OGM's underpinnings during this time will inform theoretical accounts of OGM and aid identification of clinical intervention targets. The primary aim of the current paper, therefore, was to test the CaR-FA-X model in a community adolescent sample across four annual assessment points. Moreover, in light of research with adults that found differential associations between the CaR-FA-X mechanisms and OGM as a function of depressive mood 
(Sumner et al., 2014), we further investigated the impact of early depression levels on the model's applicability to understanding OGM in youth.

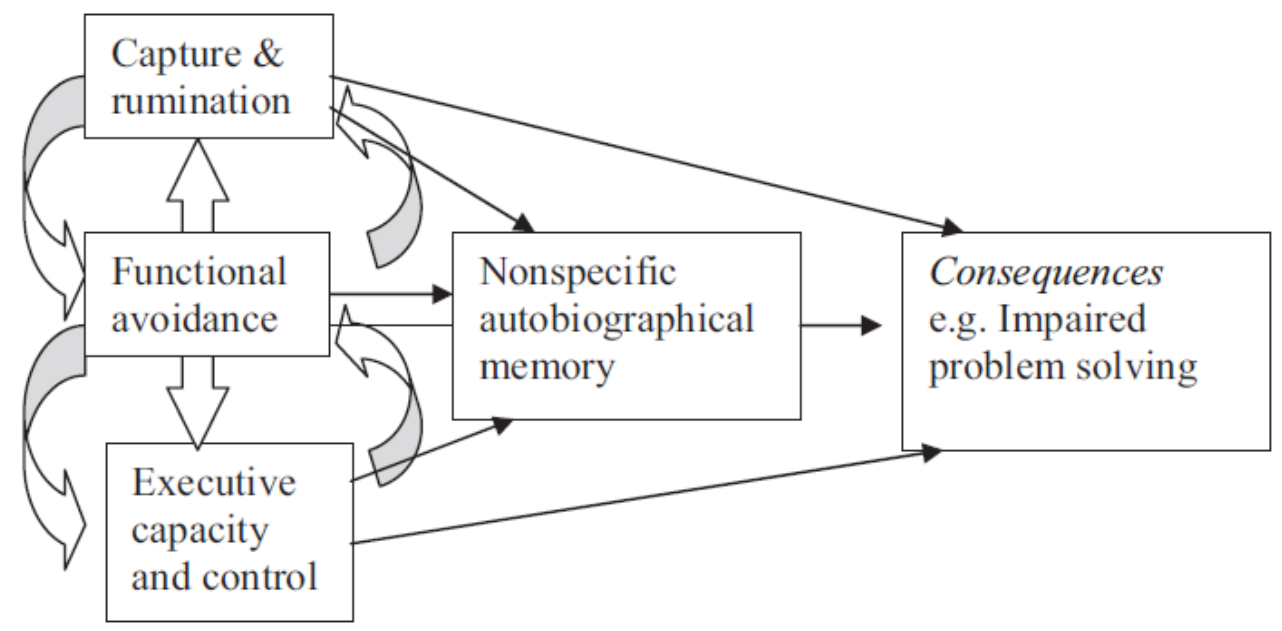

Figure 1. The CaR-FA-X Model. Three mechanisms (capture and rumination, functional avoidance and executive capacity and control) impact psychological functioning (e.g., problem solving ability) either directly, or through their independent or joint effects on autobiographical memory specificity (permission for reproduction of image granted by J. M. G. Williams; Williams et al., 2007, p. 141).

The CaR-FA-X model of OGM (Williams et al., 2007) elaborates on Conway and Pleydell-Pearce's (2000) Self Memory System (SMS) model of autobiographical memory. According to the SMS, autobiographical memory is organized hierarchically, such that when asked to retrieve a specific memory, an individual must carry out a generative retrieval search, navigating from general representations at the top to event-specific representations at lower levels. The three $C a R-F A-X$ model mechanisms are proposed to interfere with the generative retrieval search in different ways (Williams et al., 2007). First, Capture and Rumination (CaR) occurs when cue word presentation activates abstract self-relevant information, such as self-beliefs (e.g., brave = "I am not a brave person"), which captures the individual's attention. Rather than moving down the SMS hierarchy and generating a specific memory, the individual engages in rumination, resulting in premature termination of the generative retrieval search at a general level. Second, the Functional Avoidance (FA) mechanism refers to termination of the memory search at a general level to avoid potential event-related negative affect (e.g., avoiding thinking about the details and emotions related to a negative social encounter). Avoiding negative emotions negatively reinforces overgeneral 
retrieval, which, although initially adaptive, may develop over time into a passive and inflexible affect regulation strategy. And last, reduced Executive Control $(X)$, in the form of poor inhibition of irrelevant information during the memory search and/or working memory deficits, may also cause premature termination of the generative retrieval search at a general level.

A key aspect of the CaR-FA-X model is that OGM may initially be part of an adaptive emotion regulation strategy repertoire when used flexibly in response to negative experiences (Hermans et al., 2005). For some people, however, OGM may turn into an inflexible and habitual style of memory retrieval that has adverse consequences for cognitive functioning (Williams et al., 2007). One implication is that OGM relates to the CaR-FA-X model mechanisms differently across populations with different psychopathological profiles (Sumner et al., 2014; Williams et al., 2007). For example, at chronic levels of depressed mood, when emotion regulation is low, avoidance of negative emotional material - by means of truncating the retrieval search at a general level - may have a stronger reinforcing effect. For individuals from non-clinical samples manifesting low distress, however, OGM may be driven by other processes. For example, adults report that retrieval of recurrent event memories can serve adaptive social functions by highlighting the value of specific social relationships (Waters, Bauer, \& Fivush, 2014).

Research on OGM in adults with elevated depressive symptoms provides robust support for the capture and rumination and executive function mechanisms of the Car-FA-X model (see Sumner, 2012, for a review). A significant limitation of past research, however, is that most studies have focused on single mechanisms only. Adopting a cross-sectional design, Sumner et al. (2014) were the first researchers to assess all three components of the model concurrently. They reported that for people with major depression, increased rumination predicted higher concurrent levels of OGM at low levels of executive control. In contrast, for a non-depressed control group, increased rumination predicted OGM at high levels of executive control. Avoidance was not associated with OGM in either group. Consistent with Williams et al.'s (2007) proposal, these findings highlight that mechanisms can exert effects in interaction. They suggest also that the mechanisms may function differently across groups of people with varying levels of depression symptoms, as has been reported by other research (e.g., Ganly et al., 2016; Smets, Griffith, Wessel, Walschaerts, \& Raes, 2013).

Findings with youth provide some support, albeit nuanced, for the relationship between OGM and each of the CaR-FA-X factors. For example, Park et al. (2004) found a cross-sectional positive association between rumination and numbers of OGMs, but this 
relationship was limited to adolescents with first episode major depression (ages 12 to 17 years) and to memories provided in response to negative but not positive cues. In contrast, they found a non-significant relationship between rumination and the numbers/proportions of OGMs reported for individuals in the non-depressed control condition. Similarly, focusing on hospitalized children who had experienced an accidental injury (ages 7 to 17 years), Hitchcock et al. (2014b) reported that the association between rumination and OGM was nonsignificant. In terms of executive function, findings are also mixed. One study reported an association between lower inhibitory control (as a measure of executive function) and greater OGM in a community sample of youth ages 9 to 13 years (Raes et al., 2010). Another study (Valentino, Bridgett, Hayden, \& Nuttall, 2012) did not replicate this finding in a group of young people in inpatient psychiatric care (ages 7 to 17 years) who were experiencing a range of psychological difficulties (e.g., mood disorders, PTSD, behavioral problems), and instead reported a negative association between working memory capacity and frequencies of OGM. Research investigating the functional avoidance mechanism has predominantly focused on youth who have experienced trauma (e.g., Brennen et al., 2010), and only two studies have investigated the relationship between self-reported avoidance tendencies and OGM (Kuyken, Howell, \& Dalgleish, 2006; Stokes et al., 2004). As findings were mixed, strong conclusions about the role of self-reported avoidance cannot be drawn.

Two studies have investigated whether the model mechanisms predict OGM longitudinally (Hitchcock et al., 2014b; Rawal \& Rice, 2012a). Consistent with the CaR-FA$\mathrm{X}$ model, these studies also investigated possible interactions between mechanisms. In a longitudinal study of adolescents (ages 10 to 18 years) at familial risk of depression, Rawal and Rice (2012a) found that higher levels of rumination and lower levels of executive control interacted to predict OGM at a six-month follow-up. While Hitchcock et al. (2014b) did not find this relationship in community adolescents (ages 7 to 17 years) at a 12-month follow-up, greater working memory capacity was associated with lower proportions of OGM in older, but not younger, adolescents.

In summary, the mechanisms of rumination and executive function have received some support, but more research is needed, and very little work has focused on the role of the avoidance mechanism. The conclusions that can be drawn from current research are also limited by variability in the samples, particularly with respect to the age of participants and the nature and severity of their psychological symptomatology. Several factors seem to moderate the findings of significant associations. In particular, it appears that the model better accounts for OGM in clinical than non-clinical youth populations. One possible explanation 
may be that in clinical samples, existing psychological vulnerabilities moderate the degree to which the CaR-FA-X processes precipitate OGM (Crane et al., 2016; Rawal \& Rice, 2012a; Smets et al., 2013). At times of low mood or distress, when emotion regulation is impaired and inhibitory control compromised (Joormann \& Gotlib, 2010), a person may ruminate more and thus be more susceptible to Capture and Rumination errors (Raes, Schoofs, Griffith, \& Hermans, 2012; Williams et al., 2007). With increasing distress, avoidant cognitions and behaviours increase, also (Ottenbreit \& Dobson, 2004). In contrast, at low levels of distress, individual differences in OGMs reported by youth may be better accounted for by other factors, such as reduced motivation to engage in the task (Crane et al., 2016).

Cue valence may also moderate the relationship between the model's mechanisms and OGM, with some research reporting associations for OGMs generated in response to negative cue words only (Park et al., 2004). This result may suggest that OGM as an affect regulation strategy is at first limited to negative cue words, but generalizes to positive cues as psychopathology increases over time. Initially, OGM may emerge when young people attempt to minimize the emotional impact of remembering negative past events. Over time, as OGM retrieval becomes more inflexible, and distress increases, recall of a wider range of memories - regardless of emotional valence- becomes disrupted (Kuyken \& Dalgleish, 2011).

In conducting a test of the full CaR-FA-X model, the current study addressed several important gaps in the literature. In particular, our four annual waves of data for adolescents who averaged 13 years of age at Time 1 spanned the period of significant increases in psychopathology (Costello, Mustillo, Erkanli, Keeler, \& Angold, 2003) and associated cognitive processes such as rumination (Jose \& Brown, 2008) and executive functioning (Anderson, Anderson, Northam, Jacobs, \& Catroppa, 2001). At each time point, participants completed measures of the three CaR-FA-X factors and depression. This method allowed us to not only test the CaR-FA-X model in its entirety, but also to investigate possible differences in the model's applicability to groups of young people who report different patterns of early depressive symptomatology. We operationalized depression severity as trajectory of depression symptom growth across the four time points, and compared two groups of participants: one group with low, yet increasing, depression levels across the four years, and one group with higher and increasing levels. We chose this approach because depression levels tend to fluctuate across adolescence, and in light of the theoretical notion that flexibility of OGM use may reduce as a function of prolonged adversity (Hermans et al., 2008 b), we wanted to identify youth with elevated depression levels across time. We aimed to 
test, therefore, whether the CaR-FA-X processes, individually and in interaction, would function differentially across groups of young people with varying levels of depression symptoms.

As our first hypothesis, we predicted that in the higher depression group, higher levels of rumination and avoidance and reduced executive control (operationalized as inhibitory control) would predict higher OGM (operationalized as higher proportions of overgeneral memories and lower proportions of specific memories) over time, either individually or in interaction, and that associations would be stronger for memories reported to negative cues. Due to a lack of research testing multiple mechanisms concurrently, we did not make specific predictions about interaction effects. As our second hypothesis, we expected that, for the lower depression group, higher OGM would not be predicted by the CaR-FA-X processes. Finally, to strengthen any conclusions drawn from our subgroup findings and to discern whether patterns of findings across high and low subgroups are characteristic of only these groups or more widely evident in community youth, we also tested the model across the entire sample. As our third hypothesis, we expected that across the sample, as for the lower depression severity group, the CaR-FA-X mechanisms would not predict OGM.

\section{Method}

\section{Participants}

Participants were recruited from 14 schools in New Zealand. Initial recruitment at Time 1 involved communication with schools in the immediate Wellington urban area. We then systematically recruited further afield until a sufficient number of students agreed to participate. For follow-up, additional schools needed to be recruited as students transitioned to intermediate and secondary schools. Of the 323 (171 males, 152 females) adolescents who participated at Time 1, 288 participated at Time 2 (89.2\%), 269 at Time 3 (83\%), and 239 at Time $4(74 \%)$. The mean age was 12.83 years $(S D=1.18)$ at Time $1,13.87$ years $(S D=1.16)$ at Time 2, 14.74 years $(S D=1.05)$ at Time 3 , and 15.87 years $(S D=1.00)$ at Time 4 . Boys were significantly older at Time $1(M=13.10, S D=1.25)$ than girls $(M=12.54, S D=1.01)$, $t(318.27)=4.46, p<.001,95 \%$ BCa CI [3.67, 9.84]. Parental consent and student assent was obtained separately for each wave, allowing for the option of withdrawal at each follow-up point. The majority of participants identified as New Zealander or European New Zealander (80.1\%). The remaining participants identified as Mãori (5.3\%), Asian (7.9\%), Pacific Islanders (3.4\%) or Other (3.4\%). Although, across schools, low to high socio-economic backgrounds were represented, the majority of the children were of middle socio-economic 
background. This research was approved by the School of Psychology Human Ethics Committee, under delegated authority to the Victoria University of Wellington's Ethics Committee.

\section{Measures}

Depression. Participants completed the 12-item Children's Depression Inventory-2 Self-Report Short Version (CDI-2:SR[S]; Kovacs, 1985, 1992). For each item, participants are presented with three sentences per item (e.g., "I hate myself", "I do not like myself", "I like myself'), and asked to select the one response option that best corresponds to how they had been feeling over the past 2 weeks. Items are then scored on a 3-point scale $(0=n o$ symptoms, 1 = mild symptoms, 2 = severe symptoms), and a total score is computed. Internal consistency was good across the four waves (Cronbach's $\alpha$ s $=.73$ to .81 ).

Effortful Control. An index of effortful control was calculated by computing a mean score across three subscales of the Revised Early Adolescent Temperament Questionnaire (EATQ-R Short-Form; Capaldi \& Rothbart, 1992; Ellis \& Rothbart, 2001): inhibitory control (5 items; e.g., "I can stick with my plans and goals"), activation control (5 items; e.g., "I finish my homework before the due date") and attention (6 items; e.g., "I am good at keeping track of several different things that are happening around me"). Responses are made on a 5point scale ( 1 = "almost always untrue" to $5=$ "almost always true"). Higher scores indicate greater effortful control, that is, greater "ability to inhibit a dominant response to perform a subdominant response" (Rothbart \& Bates, 1998; p. 137). Effortful control and executive functioning are regarded as conceptually and empirically overlapping constructs (e.g., Bridgett, Oddi, Laake, Murdock, \& Bachmann, 2013). We used a subjective measure of selfregulation on the basis of previous research on OGM with youth (Raes et al., 2010). Across the four data collection waves, superscale Cronbach's alphas ranged from .80 to .85 .

Rumination. Participants completed an abbreviated 6-item version of the 13 -item rumination subscale of the Children's Response Styles Questionnaire (CRSQ; Abela, Rochon, \& Vanderbilt, 2000; Abela et al., 2002). Each item consists of a statement related to ruminative cognitive processes (e.g., "When I am sad, I think why can't I handle things better"), and participants report how often they engage in these thinking styles on a 4-point scale $(1=$ almost never; 2 = sometimes; 3 =often; 4 = almost always $)$. Average scale scores range from 1 to 4, with higher values representing greater rumination levels. Across the four waves of data collection, Cronbach's alphas ranged from .77 to .85 . 
Avoidance. The Affect Regulation Scale (ARS) was devised for the current study based on Jose and Huntsinger's (2005) coping measure. Three domains were measured; emotional (e.g., "when I am sad, I avoid thinking about how I feel”) and behavioural (e.g., "when I am sad, I stay away from the person or situation that is causing the problem") avoidance, as well as denial of unfavourable circumstances (e.g., "when I am sad, I try to pretend there isn't a problem"). Participants indicated how often they engage in such response styles on a 4 -point scale $(1=$ almost never; $2=$ sometimes; $3=$ often; $4=$ almost always). In light of high correlations between subscales, the three subscales were collapsed into a 10-item avoidance measure at Times 1 and 2, and a 12-item (two items added) measure at Times 3 and 4. Higher scores indicate greater engagement in avoidant affect regulation. Cronbach's alphas ranged from .77 to .86 across the four years.

As the ARS was developed specifically for the current study, additional psychometric analyses were carried out. The measure exhibited good convergent validity, with scores across the four time points correlating significantly in expected directions with other conceptually-relevant measures. For example, correlations between the avoidance and rumination scores were significant at all time points $(r=.22$ to $.32, p \mathrm{~s}<.001)$, which is consistent with past research that investigated relationships between these constructs (e.g., Moulds et al., 2007). Moreover, longitudinal measurement invariance was demonstrated across the four time points when a Longitudinal Invariance Confirmatory Factor Analysis was carried out (additional information about this analysis can be requested from the first author).

Autobiographical Memory. The written version of the Minimal Instructions Autobiographical Memory Test (Mi-AMT; Debeer et al., 2009) assessed memory retrieval style. The Mi-AMT has been found to be more sensitive to overgenerality in non-clinical populations than the original AMT (Debeer et al., 2009). Participants were presented with 10 cue words in fixed order, alternating between positive and negative valence. The cues (happy, sad, lucky, angry, proud, lonely, excited, guilty, relaxed, scared) were obtained from previous research with youth (Hipwell et al., 2011; Raes et al., 2010). Following piloting, minor alterations were made to the test instructions. Participants were instructed that they are completing a memory test, and that, for each cue, they are requested to think of and write down a specific event of which the cue reminds them. As an example, they were provided with a correct and incorrect response to a cue word not included in the Mi-AMT, but they were not asked to complete practice items. Participants were told not to use the same memory more than once. For each cue, participants had one minute to respond. Participants provided memories in writing; our previous research suggests that, in children, mode of memory 
reporting (written versus spoken) does not predict number of overgeneral and specific memories reported (BLINDED FOR REVIEW).

Memory coding and reliability. Responses on the AMT were coded for temporal specificity using pre-established criteria (e.g., Crane et al., 2016; Griffith et al., 2012). Memories referring to single events that did not last longer than one day were coded as specific (e.g., "when I went to the cinema on Saturday"); memories referring to a category of similar events were coded as categoric (e.g., "when we go to the park"); memories referring to events that took longer than 24 hours were coded as extended (e.g., "going to Australia last year"). Several additional types of responses were errors and not analyzed further: semantic associates (verbal associations, such as "bed" in response to cue word happy); future-oriented responses (reports of events that had not yet happened, such as, "going on camp next week"); repetitions of previous responses; incomplete responses; omission (failure to provide a response). Mean inter-rater reliability across three trained raters was good overall $(\kappa=.75)$. Six indices of memory specificity, or overgenerality, were calculated. Following Griffiths et al. (2012), indices of overgenerality (categoric and extended memories) and specificity (specific memories) were computed as proportions of non-missing responses. In line with findings of cue valence effects in young people (e.g., Park et al., 2004), we also calculated separate indices of overgenerality and specificity for positive and negative cue words.

\section{Procedure}

Participants were assessed during school time in groups of approximately 10 students. Before data collection commenced, students were informed that responses were confidential and would only be traced back to individuals if responses gave concerns about their wellbeing. In that case, the school would be contacted (for individuals younger than 16 years) or the individual themselves (for individuals 16 years and older). Following informed assent, the Mi-AMT was administered. For the second part of the session, students completed self-report questionnaires. Participants were then debriefed about the purpose of the study, and invited to ask questions. Each session was of approximately 45 minutes' duration, and trained postgraduate students and research fellows carried out data collection and coding.

\section{Data Analysis}

Across the four data collection points, the total percentage of missing scores across the measures was $14.2 \%$. Little's MCAR test was statistically significant, indicating that data were not missing completely at random. To maximize power, we employed the expectationmaximization (EM) algorithm to impute missing values in SPSS Version 23. The software 
package Mplus 7 (Muthén \& Muthén, 1998-2015) was used for growth modeling analysis, and AMOS 23 (Arbuckle, 2014) for structural equation modeling. As some measures were non-normally distributed, we employed the MLR estimator for analyses in Mplus, and bootstrapping in AMOS. Hypotheses were tested using bias-corrected (BC) 99\% bootstrap confidence intervals (CI) (2000 samples). Due to the number of multiple comparisons made, parameter estimates and bootstrapped confidence intervals are only reported for findings significant at the $p<.01$ level.

Next, to identify subgroups of participants with different depression growth trajectories across the four time points, latent class growth analyses (LCGA) were carried out. Unlike conventional growth modeling analyses, this approach identifies subgroups with distinct growth curves, rather than treating the sample as coming from a single population with a single growth trajectory (Jung \& Wickrama, 2008). Models with different numbers of subgroups are compared to identify the best-fitting model with the smallest Bayesian Information Criterion (BIC), and significant Lo et al. (2011) likelihood ratio (LMR-LRT) test statistic (Jung \& Wickrama, 2008).

Prospective associations between CaR-FA-X mechanisms and OGM were tested using autoregressive cross-lagged structural equation models. This approach is suited to the current research questions because it allows simultaneous inclusion of multiple factors across multiple time points. To test the first part of our hypothesis that the CaR-FA-X factors are related to OGM in youth who experience higher, but not lower, depression over time, multigroup analyses were carried out using one of the six memory indices at a time. Computational constraints were imposed by our inclusion of main effects and interactions and also because we considered it important to allow stability coefficients to vary across groups. Therefore, separate analyses were carried out for each time lag. First we assessed main effects and then included interaction terms (three in total, each representing the product of two model mechanisms) to test whether interactions between the CaR-FA-X mechanisms predicted OGM above and beyond the main effects. Variables were allowed to covary with one another, as well as age and gender. If a coefficient was found to be statistically significant in either trajectory group, follow-up chi-square difference tests were carried out. That is, a group difference was considered significant only if the chi-square value significantly increased as a function of constraining the pathway of interest to equality across trajectory subgroups (indicating worse model fit).

For tests of prospective associations across the entire sample, single models including all four waves of data were fit for each one of the six memory indices. To identify the most 
parsimonious models, models with freely varying autoregressive pathways (associations between scores on the same measure across the three time lags) were compared to models where autoregressive pathways were constrained to be equal. To allow for estimation of timespecific prospective effects, cross-lagged pathways were allowed to vary freely (e.g., associations between rumination and OGM from Time 1 to Time 2, Time 2 to Time 3, and Time 3 to Time 4 were not constrained to be equal).

\section{Results}

\section{Descriptive Statistics}

The study variables' means and standard deviations are presented in Table 3 for the entire sample, and in Table 5 for the depression trajectory groups. Correlation analyses (Table 4) show that the three CaR-FA-X model factors were not consistently associated with proportions of overgeneral or specific memories across the four waves of data collection. Due to the large number of comparisons made, we adopted a more stringent criterion of statistical significance. At the $p<.01$ level, six correlations were statistically significant. Higher levels of rumination at Times 1, 2, and 3, were associated with lower proportions of specific memories at Time 2. Higher levels of avoidance at Time 3 were associated with higher proportions of overgeneral memories at Time 4, and higher effortful control at Time 2 was associated with lower proportions of overgeneral memories and higher proportions of specific memories at Time 2 . 
Table 3

Descriptive Statistics of the Study Variables $(N=323)$

\begin{tabular}{lcccc}
\hline & Wave 1 & Wave 2 & Wave 3 & Wave 4 \\
\hline Prop. OGM & $.23(.19)$ & $.19(.17)$ & $.19(.15)$ & $.21(.14)$ \\
\multicolumn{1}{c}{ Neg. cues } & $.26(.25)$ & $.23(.22)$ & $.24(.21)$ & $.26(.19)$ \\
\multicolumn{1}{c}{ Pos. cues } & $.20(.21)$ & $.15(.19)$ & $.14(.16)$ & $.15(.16)$ \\
Prop. SPM & $.66(.23)$ & $.69(.20)$ & $.72(.18)$ & $.69(.17)$ \\
\multicolumn{1}{c}{ Neg. cues } & $.62(.28)$ & $.64(.25)$ & $.66(.23)$ & $.62(.22)$ \\
$\quad$ Pos. cues & $.70(.26)$ & $.75(.23)$ & $.77(.20)$ & $.76(.20)$ \\
CRSQ & $1.99(0.66)$ & $2.01(0.72)$ & $2.07(0.65)$ & $2.17(0.61)$ \\
ARS & $2.23(0.51)$ & $2.20(0.47)$ & $2.18(0.46)$ & $2.28(0.47)$ \\
EATQ-R & $3.38(0.56)$ & $3.39(0.58)$ & $3.29(0.52)$ & $3.23(0.50)$ \\
CDI-2 & $4.39(3.11)$ & $4.62(3.49)$ & $5.60(3.67)$ & $6.07(3.49)$
\end{tabular}

Note. Prop. OGM = Proportion of overgeneral autobiographical memories (sum of categoric and extended memories); Prop. SPM = Proportion of specific autobiographical memories; CRSQ = Children's Response Styles Questionnaire; ARS = Affect Regulation Scale; EATQ$\mathrm{R}=$ Early Adolescent Temperament Questionnaire; CDI-2 = Children's Depression Inventory 2. 
Table 4

Pearson's Correlations Between CaR-FA-X Model Factors and Primary Memory Indices (Findings Significant at the .01 Level in Bold)

\begin{tabular}{|c|c|c|c|c|c|c|c|c|}
\hline & \multicolumn{4}{|c|}{ Prop. OGM } & \multicolumn{4}{|c|}{ Prop. SPM } \\
\hline & $\mathrm{T} 1$ & $\mathrm{~T} 2$ & T3 & $\mathrm{T} 4$ & T1 & $\mathrm{T} 2$ & T3 & $\mathrm{T} 4$ \\
\hline CRSQ T1 & .05 & .09 & .02 & .01 & -.10 & $-.18 * *$ & -.03 & .11 \\
\hline CRSQ T2 & .05 & .04 & .04 & .01 & -.06 & $-.15 * *$ & -.09 & .04 \\
\hline CRSQ T3 & .04 & .08 & .05 & -.01 & -.06 & $-.16 * *$ & -.07 & .05 \\
\hline CRSQ T4 & .05 & .02 & .01 & -.03 & -.03 & -.07 & -.01 & .04 \\
\hline ARS T1 & .10 & -.03 & -.05 & .10 & -.12 & -.06 & .03 & -.02 \\
\hline ARS T2 & .03 & -.02 & .02 & .09 & -.03 & -.07 & -.05 & -.06 \\
\hline ARS T3 & .03 & .04 & .01 & $.17 * *$ & -.02 & -.11 & -.05 & -.12 \\
\hline ARS T4 & -.05 & -.07 & -.12 & -.04 & .02 & -.03 & .06 & .07 \\
\hline EATQ-R T1 & -.04 & -.12 & -.09 & -.07 & .07 & .12 & .10 & .05 \\
\hline EATQ-R T2 & -.01 & $-.15 * *$ & -.12 & -.01 & .04 & $.17 * *$ & .13 & -.02 \\
\hline EATQ-R T3 & -.05 & -.12 & -.09 & .02 & .06 & .13 & .08 & -.02 \\
\hline EATQ-R T4 & -.07 & -.08 & -.05 & .05 & .06 & .08 & .03 & -.05 \\
\hline
\end{tabular}

Note. Prop. OGM = Proportion of overgeneral autobiographical memories (sum of categoric and extended memories); Prop. SPM = Proportion of specific autobiographical memories; CRSQ = Children's Response Styles Questionnaire; ARS = Affect Regulation Scale; EATQ-R $=$ Early Adolescent Temperament Questionnaire.

$* * p<.01$.

A repeated measures ANOVA was carried out to investigate change in mean scores over the four time points. Mauchly's tests indicated that the assumption of sphericity had been violated for each measure, $\chi^{2}(5)=12.04$ to $121.98, p s=.030$ to $p<.001$. Degrees of freedom were thus corrected using the Greenhouse-Geisser estimates of sphericity $(\varepsilon=.85$ to .98). The main effect of time was significant for depression, $F(2.92,943.01)=44.23, p$ $<.001, \eta_{\mathrm{p}}{ }^{2}=.121$, with mean depression scores increasing over time (Time 3 and Time 4 significantly greater than Time 1 and Time 2). Similarly, rumination scores increased over time, $F(2.81,903.25)=11.08, p<.001, \eta_{\mathrm{p}}^{2}=.033$. Only Time 4 differed significantly from the other time points, however, and no other significant time point differences were found. For the avoidance measure, scores were relatively stable across the first three waves before increasing significantly at Time 4 (relative to Time 2 and Time 3$), F(2.91,936.31)=4.32, p$ $=.005, \eta_{\mathrm{p}}{ }^{2}=.013$. Effortful control scores were relatively stable across the first two time 
points, then decreased at Time 3 and 4 , with scores at Time 4 being significantly lower than scores at the other time points, $F(2.53,816.09)=16.13, p<.001, \eta_{\mathrm{p}}{ }^{2}=.048$. Proportion of OGMs significantly decreased at Time 2 and $3, F(2.73,878.69)=6.05, p=.001, \eta_{\mathrm{p}}{ }^{2}=.018$. Proportions of specific memories remained relatively stable, with significant differences only between Time 1 and Time 3 scores, $F(2.79,897.62)=5.76, p=.001, \eta_{\mathrm{p}}{ }^{2}=.018$.

\section{Do Depression Growth Classes Moderate the CaR-FA-X Model's Prediction of OGM?}

To determine the number of distinct subgroups of students with varying trajectories of change on the depression measure across the four time points, latent class growth models with different numbers of classes were compared in terms of model fit. First, we carried out LCGA analyses to identify two distinct depression trajectory classes. Specification of a threeclass model demonstrated superior model fit relative to a two-class model, as evidenced by lower BIC and significant LMR-LRT fit statistics. Specification of a four-class model resulted in a non-significant LMR-LRT and only a marginal reduction in BIC, rendering the three-class solution as optimal. The three subgroups identified were: a low-increasing group $(N=176, I=2.53, S=.40, p \mathrm{~s}<.001)$; a medium-increasing group $(N=121, I=5.43, S$ $=.40, p \mathrm{~s}<.001)$; and a higher-stable group $(N=26, I=10.60, S=.64, p<.001$ and $p=.05$, respectively). Considering that the higher-stable group represented less than $10 \%$ of the sample $(N=26)$, we collapsed the small higher-stable group with the medium-increasing group to form a single medium-higher group $(N=147)$; this group was compared to the lowincreasing group $(N=176)$ in subsequent analyses.

Table 5 presents descriptive statistics for the depression trajectory subgroups. It should be noted that depression trajectory group labels (low and medium/high) are relative. According to the CDI-2 scoring guide, the medium/high subgroup mean depression levels, although increasing over time, would be considered average at Time 1 and 2, and higher average/elevated at Time 3 and 4. The low depression subgroup would be considered average or lower at all time points.

A repeated measures ANOVA was run to test for differences in proportions of OGM and specificity reported across the two trajectory subgroups, including covariates of age and gender. Levene's tests of equality of variances were non-significant for each measure across all four time points. Tests of main effects for depression growth group membership were nonsignificant for both proportions of OGMs and specific memories, respectively $F \mathrm{~s}(1,319)=$ 0.34 and $0.33, p s=.560$ and .569 . 
Table 5

Descriptive Statistics of the Study Variables for Low $(N=176)$ and Medium/High $(N=147)$ Depression Growth Subgroups

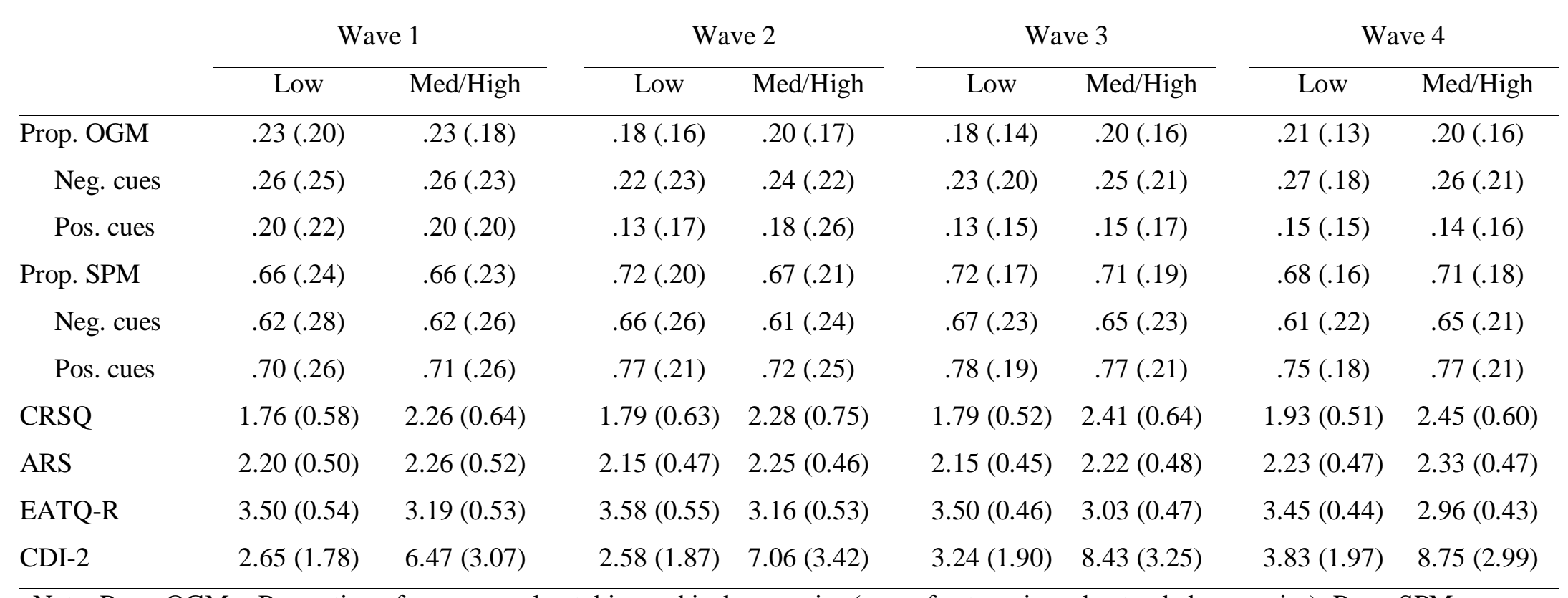

Note. Prop. OGM = Proportion of overgeneral autobiographical memories (sum of categoric and extended memories); Prop. SPM =

Proportion of specific autobiographical memories; CRSQ = Children's Response Styles Questionnaire; ARS = Affect Regulation Scale;

EATQ-R = Early Adolescent Temperament Questionnaire; CDI-2 = Children's Depression Inventory 2. 
To test our first two hypotheses, main effect models were first tested, and then models including interaction terms. Across the 18 cross-lags examined, four main effect differences were found using chi-square difference tests. As can be seen in Table 6, increased avoidance at Time 3 predicted higher proportions of overgeneral memories at Time 4 in the medium/high depression growth group $(\beta=.28, p=.001$, BC 99\% CI $[.109, .445])$ but not the lower growth group $(\beta=.05, p=.576, \mathrm{BC} 99 \% \mathrm{CI}[-.187, .274])$. Follow-up valence effect tests suggested that increased avoidance predicted higher proportions of overgeneral memories to negative cue words in the medium/high depression growth group $(\beta=.32, p$ $=.001, \mathrm{BC} 99 \% \mathrm{CI}[.129, .502])$ but not the low depression growth group $(\beta=-.12, p=.205$, BC 99\% CI $[-.322, .121])$. No significant group difference was found for prospective associations between avoidance and proportions of overgeneral memories to positive cue words. Increased avoidance at Time 3 also predicted lower proportions of specific memories at Time 4 in the medium/high depression growth group $(\beta=-.25, p<.001, \mathrm{BC} 99 \% \mathrm{CI}[-$ $.397,-.074])$ but not in the lower growth group $(\beta=.01, p=.947$, BC 99\% CI $[-.215, .225])$. Follow-up valence effect tests suggested that increased avoidance predicted lower proportions of specific memories to negative cue words in the medium/high depression growth group $(\beta=-.25, p=.001$, BC 99\% CI [-.411, -.075]), but not the low depression growth group $(\beta=.13, p=.115, \mathrm{BC} 99 \% \mathrm{CI}[-.084, .356])$. No significant associations were found for specific memories to positive cue words. When including interaction terms as predictors of overgeneral memory indices, no significant interaction effect differences were found across depression trajectory groups using the chi square difference test. 
Table 6

Standardized Beta Coefficients and Bias-corrected 99\% Bootstrapped Confidence Intervals for Avoidance (T3) Predicting OGM Proportions (T4) Across Entire Sample and Depression Growth Subgroups (Findings Significant at the .01 Level in Bold)

\begin{tabular}{|c|c|c|c|c|c|c|}
\hline & Prop. OGM & Prop. OGM neg & Prop. OGM pos & Prop. SPM & Prop. SPM neg & Prop. SPM pos \\
\hline \multirow[t]{2}{*}{ Entire sample } & .18 & .11 & .16 & -.13 & -.07 & -.11 \\
\hline & {$[.045, .315]$} & {$[-.040, .260]$} & {$[.023, .297]$} & {$[-.263, .001]$} & {$[-.211, .072]$} & {$[-.250, .025]$} \\
\hline \multirow[t]{2}{*}{ Low } & .05 & -.12 & .17 & .01 & .13 & -.08 \\
\hline & {$[-.187, .274]$} & {$[-.322, .121]$} & {$[-.057, .377]$} & {$[-.215, .225]$} & {$[-.084, .356]$} & {$[-.318, .143]$} \\
\hline \multirow[t]{2}{*}{ Med/High } & .28 & .32 & .14 & -.25 & -.25 & -.16 \\
\hline & {$[.109, .445]$} & {$[.129, .502]$} & {$[-.033, .299]$} & {$[-.397,-.074]$} & {$[-.411,-.075]$} & {$[-.342, .042]$} \\
\hline
\end{tabular}

Note. Prop. OGM = Proportion of overgeneral autobiographical memories (sum of categoric and extended memories); Prop.

SPM $=$ Proportion of specific autobiographical memories; neg $=$ negative cues; pos $=$ positive cues . 
In summary, our first hypothesis that the CaR-FA-X mechanisms would predict OGM in youth with elevated depressive symptoms was partially supported. Specifically, the medium/high trajectory group showed that increased avoidance at Time 3 predicted higher proportions of OGMs, and also lower proportions of specific memories, at Time 4, in particular to negative cue words. The second prediction that the model mechanisms would not predict OGM in the lower depressive symptom group was supported.

\section{Does the CaR-FA-X Model Predict OGM Across the Entire Sample?}

Prior to testing associations between the three CaR-FA-X model components and OGM, we identified best-fitting models for each of the six memory indices. Step-wise relaxation of autoregressive parameters only improved model fit when associations between scores of effortful control were allowed to vary freely across the four waves. Consequently, the most parsimonious models entailed constrained autoregressive pathways across the three time lags for all measures with the exception of effortful control (final models fits: $\chi^{2}(81, N=$ $323)=241.92$ to $266.00(p \mathrm{~s}<.001), C F I \mathrm{~s}=.91$ to .92 , and $R M S E A \mathrm{~s}=.08)$.

To test the third hypothesis that the CaR-FA-X mechanisms would not predict OGM across the entire sample, we first examined models' main effects. Out of the 54 main effect tests (three cross-lags for each of the three time lags, tested separately using one of the six memory indices at a time), only two significant findings emerged at $p<.01$. As can be seen in Table 6, increased avoidance at Time 3 predicted higher proportions of overgeneral memories at Time $4(\beta=.18, p=.001, \mathrm{BC} 99 \% \mathrm{CI}[.045, .315])$. This finding was qualified by cue valence, with increased avoidance at Time 3 predicting higher proportions overgeneral memories to positive cue words at Time 4 ( $\beta=.16, p=.003$, BC 99\% CI $[.023, .297])$, but not negative cue words $(\beta=.11, p=.052$, BC 99\% CI $[-.040, .260])$. Next, interaction terms were included with the main effects, and tested across the six models. No significant interaction effects were found. Thus, our third hypothesis that across the entire community sample the model mechanisms would not predict OGM was largely supported. Unexpectedly, avoidance predicted increases in OGM to positive cue words, but only from Time 3 to Time 4.

\section{Discussion}

This study is the first to test Williams et al.'s (2007) CaR-FA-X model of OGM in its entirety across four time points in a sample of non-clinical youth. We hypothesized that differences in associations between model mechanisms and OGM would emerge across groups reporting lower or higher psychological vulnerability, operationalized as low or 
medium/high trajectories of depression growth. Specifically, we predicted that for youth with higher depression levels, the three model mechanisms (increased rumination and avoidance and reduced executive control) would predict OGM recall independently, or in interaction, across four annual time points. Across the entire sample, however, as for the low depression growth group, we expected that the CaR-FA-X mechanisms would not predict OGM. Both predictions were partially supported. First, increased avoidance predicted higher proportions of overgeneral and lower proportions of specific memories to negative cue words only in the medium/high depression trajectory group, but these findings were limited to the third time lag. Second, none of the CaR-FA-X model mechanisms consistently predicted overgeneral memory across the entire sample or in the low depression group. Collectively, findings highlight that the CaR-FA-X model mechanisms, either independently or in interaction, do not explain significant variability in OGM in community youth. While the avoidance mechanism may precipitate OGM to some degree, findings were only noted for a single time period and as a function of higher depression levels.

Our finding that avoidance predicted OGM only for people in the high depression group is consistent with the notion that symptom severity or clinical status moderates associations between the CaR-FA-X mechanisms and OGM (Sumner et al., 2014; Williams et al., 2007). Specifically, in youth with consistently higher depressive symptom levels across time, increased avoidance at Time 3 predicted increased overgenerality and reduced specificity at Time 4. Although most research on the functional avoidance mechanism in youth has focused on associations between OGM and past trauma, our findings are consistent with one cross-sectional report of associations between self-reported avoidant tendencies and higher OGM for depressed youth (Stokes et al., 2004). We extend this research, however, in demonstrating longitudinal increases in OGM as a function of increased avoidance in youth with elevated depression levels, while controlling for established covariates. Thus, although our findings are restricted to one time period only, they suggest that youth with a tendency to engage in avoidant coping may also use overgeneral remembering as a means of reducing the likelihood of experiencing distressing memories and associated affect (Hermans et al., 2005; Moore and Zoellner, 2007; Williams, 1996; Williams et al., 2007).

Associations between increased avoidance and higher proportions of OGMs were stronger for memories to negative cues than to memories to positive cues. In contrast, studies with adults with clinical-level difficulties often find OGM in response to negative and positive cue words (Williams et al., 2007). The current finding may suggest that in youth with enduring elevated depressive symptoms, OGM initially serves to regulate affect associated 
with information elicited by negatively-valenced cue words. At early stages of the development of this coping mechanism, therefore, young people with elevated depression levels may selectively avoid retrieval of negative memories to regulate their emotional experience (Kuyken \& Dalgleish, 2011). With increasing levels of psychopathology, however, OGM, as a learned affect regulation strategy, may generalize to positive cue words also (Williams et al., 2007).

Particularly interesting was that the longitudinal relationship between avoidance and overgeneral memory emerged despite the fact that levels of reported avoidance and OGM did not differ significantly across depression subgroups. That is, young people in the medium/high depression growth group were not more avoidant, nor more overgeneral, than adolescents with lower depression trajectories. These findings suggest that avoidance and retrieval of overgeneral memories have a different relationship with each other, and may serve different functions, for individuals with higher versus lower levels of depression. Other research, also, suggests this possibility (e.g., Ganly et al., 2016). For young people with lower depression, using OGM flexibly might help to avoid negative feelings in the short term. Indeed, some findings suggest that in non-clinical populations, higher OGM can even serve a protective function and be associated with reductions in negative affect (e.g., Gutenbrunner et al., 2017; Raes et al., 2003). At increasing and chronic levels of distress, however, overgeneral remembering may become inflexible and be a product of, and further exacerbate, psychological difficulties. If OGM represents one facet of a broader avoidant coping style, clinical interventions with depressed youth may benefit from addressing biases in autobiographical remembering alongside other vulnerabilities (Hitchcock, Werner-Seidler, Blackwell, \& Dalgleish, 2017).

Increased rumination and reduced executive control did not predict higher OGM in either subgroup or the entire sample. This is inconsistent with the CaR-FA-X model and other theories that focus specifically on diminished cognitive regulation resources and increased ruminative self-processing as causes of OGM (Dalgleish et al., 2007; Watkins \& Teasdale, 2004). The reasons for this finding are unclear but we can speculate on potential factors. In Rawal and Rice's (2012a) one-year longitudinal study demonstrating predictive relationships between (higher) rumination and (lower) executive function and memory specificity, avoidance was not measured. A particular strength of the current study, however, is that our findings enabled conclusions about the role of avoidance while controlling for rumination and executive control (and vice versa), permitting greater clarity of the relative influence of each. Our self-report measure for the executive control mechanism further differed from the 
measure adopted by Rawal and Rice, which was the Block Design test of the Wechsler Intelligence Test for Children (Wechsler, 2004). Given the complexity of the executive control construct (Duckworth \& Steinberg, 2015), differences in conceptualization and measurement likely contribute to inconsistencies in findings in this research area. Finally, with regard to the rumination mechanism, although we refer to trajectory groups as low or medium/high, our overall levels of depressive symptoms in the sample were relatively low, particularly at early time points. Perhaps negative self-beliefs in the current sample were still less well established than in other research samples with greater depression, thereby reducing likelihood of Capture and Rumination errors.

The finding that the three CaR-FA-X mechanisms did not consistently predict OGM across the entire sample mirrors past research with non-clinical youth populations (e.g., Kuyken et al., 2006; Smets et al., 2013). The current study’s findings extend past, predominantly cross-sectional, research by highlighting that the three CaR-FA-X mechanisms do not account for significant variability in OGM across four annual assessment points in this group of community adolescents. Two significant associations were found across the entire sample. First, increased avoidance at Time 3 predicted higher OGM at Time 4, but this finding was likely driven by the association found in the medium/high depression growth subgroup. In contrast, the finding that higher avoidance at Time 3 predicted higher OGM to positive cue words at Time 4 across the entire sample was not moderated by depression growth. This result should be interpreted with caution, however; relative to the other findings, the effect size was small and the confidence interval's lower bound included values close to zero.

If not the CaR-FA-X mechanisms, what then underlies or drives OGM in non-clinical youth? At least three explanations are possible. First, although research on alternative underlying mechanisms is lacking, lack of motivation or willingness to disclose sensitive information when responding to the AMT may play a role (Crane et al., 2016). Further, research investigating the development of autobiographical memory also suggests that retrieval of some general memories when asked to retrieve a specific memory is normative in young people (Peterson et al., 2016), perhaps as a result of early memory socialization practices between mother and child which can shape style of recalling past events (Valentino et al., 2014). Third, it is also possible that OGM serves adaptive functions in children as has been reported with adults (e.g., Waters et al., 2014); for example, memories with a social component (e.g., "family holidays") can support the development of a healthy relational sense of self (Wang, 2004). For a more comprehensive understanding of OGM's etiology in 
youth, future research needs to 1) test CaR-FA-X model in its entirety longitudinally in samples with different forms and levels of psychopathology, and 2) investigate a broader range of mechanisms, at various ecological levels (Valentino, 2011).

Other findings from this same sample (BLINDED FOR REVIEW) and other work (see Hamlat et al., 2015) demonstrate that rumination interacts with overgeneral memory to predict psychopathology in youth. Together with the current results, these findings paint a complex picture of the longitudinal interrelationships among overgeneral memory, avoidance, rumination, and psychopathology in the context of increasing risk. Beyond the current study's time points, avoidant coping, over time, may foster retrieval inflexibility in youth with elevated low mood. As overgeneral retrieval becomes more rigid and distress increases, cognitive resources may become compromised and negative general self-representations more accessible and well-established. As a result, capture and rumination errors and OGM may increase, both maintaining and exacerbating psychological distress. Indeed, research with adolescents has found avoidance to predict increases in rumination over a short delay, which, in turn, mediated associations between avoidance and subsequent increases in low mood and anxiety (Dickson, Ciesla, \& Reilly, 2012).

The current research has several limitations. First, the large number of analyses performed here may have inflated Type 1 error rate. To compensate for this strategy, we adopted a more stringent significance threshold, thus reducing the risk of falsely rejecting the null hypothesis. Second, while we could have investigated possible gender effects, this issue was not a primary research question for this paper, and group difference testing would have increased computational burden significantly. We did include gender and age as covariates in all analyses, however. Third, our analyses did not account for young peoples' language skills or IQ, which may have impacted retrieval of memories on the AMT (e.g., Hipwell et al., 2011). Fourth, measurement limitations must be acknowledged. That is, inconsistencies in how past research conceptualized and operationalized the three mechanisms make it difficult to compare studies' findings directly and thus limit their generalizability. For a more comprehensive understanding of associations between the three CaR-FA-X mechanisms and OGM in youth, future research should test the model using a range of construct measures (e.g., assess both capture and rumination, include measures of both trauma and functional avoidance, and adopt measures of executive function that tap into a range of executive control capacities). Finally, participants in the current study were predominantly of New Zealand European ethnicity and of middle-class social-economic origin, which, in the context of 
research demonstrating consistent cultural influences on autobiographical memory (Wang, 2004), limits the generalizability of our findings.

In conclusion, the current findings suggest that the CaR-FA-X model exhibits limited efficacy in non-clinical adolescents. While a few supportive findings were identified, relationships were transient and qualified by levels and growth of depressive symptoms. In youth reporting higher trajectories of depression across the four time points, increased avoidance predicted increases in OGM, but only from Time 3 to Time 4. These findings suggest that the CaR-FA-X functional avoidance mechanism may, to some degree, explain OGM in youth at higher risk for future adversity, but only under certain conditions, such as when depression levels have persisted over an extended period of time and reach a critical threshold. In youth with lower depression trajectories, individual variability in OGM may be better accounted for by processes other than the CaR-FA-X mechanisms. 


\section{Chapter 4}

Study 3: Autobiographical Memory Specificity and Detail: Investigating Longitudinal Associations with Psychopathology in Youth

The previous chapters filled two important gaps in the literature on OGM in community youth. First, whether OGM represents a marker of vulnerability for early psychopathology, and second, whether the predominant theoretical account of OGM - the CaR-FA-X model (Williams et al., 2007) — has explanatory utility in this population. In the current, and final, empirical chapter of this thesis I address a third important research question that builds on Studies 1 and 2. Specifically, I broaden the focus by investigating associations between OGM, psychopathology and another facet of autobiographical memory that is elicited by the AMT - event-specific (or episodic) memory detail. Indeed, young people not only differ in terms of the number of temporally specific memories they report on this measure, but also with regard to how much detail their specific event recollections contain. A recent study with community adults suggests that these two indices of performance on the AMT are cross-sectionally unrelated and may have different implications for psychological functioning (Kyung et al., 2016). Drawing on this research, the current study tested associations between memory specificity and detail on the AMT in community youth, and also examined similarities and differences in the two constructs' longitudinal associations with psychological functioning. Addressing these relatively unexplored research questions can have important implications for our understanding of relationships among different dimensions of autobiographical memory and emerging psychopathology in young people.

\section{Chapter 4 is composed of a manuscript that has been submitted to Memory:}

Gutenbrunner, C., Salmon, K., \& Jose, P. E. (2017). Episodic specificity and detail: Investigating longitudinal associations with psychopathology in youth. Manuscript submitted for publication. 


\begin{abstract}
The tendency to report fewer specific event memories on the Autobiographical Memory Test (AMT; Williams \& Broadbent, 1986) in some studies predicts psychopathology in youth. Recent findings suggest that reporting of specific memories and episodic detail (eventspecific detail such as sensory, perceptual and affective information) are unrelated in adults, and differentially predict psychological functioning (Kyung, Yanes-Lukin, \& Roberts, 2016). Despite significant increases in psychopathology during adolescence, these relationships have not been investigated in young people. We, therefore, tested associations between memory specificity, episodic detail and psychological functioning in a community sample of 96 adolescents (34 females, 62 males) across 3 annual assessment points. Reporting of specific memories was not associated with reporting of episodic detail. Moreover, longitudinal associations between memory dimensions and depression, anxiety, and rumination, were generally non-significant. We found that higher anxiety, in interaction with higher rumination, predicted reporting of fewer specific memories, and less episodic detail in specific memories to negative cue words, but these effects were transient. Overall, our findings highlight that in community youth, memory specificity, episodic detail and psychopathology are unrelated.

Keywords: autobiographical memory specificity; episodic memory detail; anxiety; depression; adolescence
\end{abstract}




\section{Introduction}

Autobiographical remembering, namely the ability to recall events from our personal past, is central to adaptive everyday functioning (e.g., Bluck et al., 2005). Biases in autobiographical remembering, conversely, are implicated in the development and maintenance of psychological difficulties (e.g., Dalgleish \& Werner-Seidler, 2014). One such bias is reduced memory specificity, which is the tendency to report general events, or events that lasted for extended periods of time, instead of specific memories (Williams \& Broadbent, 1986). For example, when provided with the cue word "sad", instead of recalling "yesterday when my friend ignored me", a person may report "when my friend ignores me" (a general category of events) or "when my friend ignored me for a week" (an extended period of time). When defined this way, reduced memory specificity captures lack of temporal specificity. This phenomenon is clinically meaningful because it has been shown to predict increases in psychopathological symptoms in children and adults, in particular depression (Hitchcock et al., 2014a; Sumner et al., 2010; Williams et al., 2007).

Despite reduced memory specificity's role in the development of psychopathology, limited research has investigated its relationship with other autobiographical memory dimensions. For example, episodic memories (i.e., memories that refer to a unique event that did not last longer than one day) can contain varying levels of episodic detail (i.e., sensory, perceptual and affective information relating to the specific event). Drawing on hierarchical models of autobiographical memory (e.g., Conway \& Pleydell-Pearce, 2000), it is generally assumed that specific memories contain more event-specific episodic details than nonspecific memories (e.g., Levine, Svoboda, Hay, Winocur, \& Moscovitch, 2002). Indeed, reduced memory specificity may predict psychopathology because people have reduced access to event-specific information, which is important for adaptive cognitive functioning (e.g., problem-solving; Jing et al., 2016).

Despite the possible role that episodic detail may play in understanding associations between reduced memory specificity and psychopathology, only one study, a cross-sectional study with an adult sample, has investigated relationships among these constructs (Kyung et al., 2016). Adolescence is critical for the onset of psychopathology, however, and relatively little is currently known about mechanisms underlying reduced memory specificity and its association with psychological difficulties in this population. We, therefore, investigated concurrent associations between memory specificity and reporting of episodic detail in a community youth sample, as well as longitudinal associations between these aspects of memory and psychological functioning (depression, anxiety, and rumination). 
The predominantly cross-sectional research with children and adolescents has established a relatively weak, yet consistent, association between reduced memory specificity and depressive symptoms (for a review, see Hitchcock et al., 2014a). Limited longitudinal research exists, and findings are mixed when variables are examined over time. Indeed, evidence suggests that low specificity is a more robust predictor of psychopathology in clinical samples (e.g., Hipwell et al., 2011) than in non-clinical populations (e.g., Crane et al., 2016). Interestingly, for typically developing adolescents, findings indicate that reduced memory specificity predicts increases in depression (Hamlat et al., 2015) and anxiety (Gutenbrunner et al., 2017), but only in conjunction with increased rumination. Other psychological vulnerabilities may therefore be implicated in relationships between memory specificity and psychopathology in community youth populations.

What makes young people who report fewer specific memories more vulnerable to developing psychopathology? As reported earlier, one possible explanation is that reduced memory specificity hinders effective problem-solving in the face of adversity due to insufficient access to information about past events (Jing et al., 2016; Williams et al., 2007). Arguably, in order to learn from specific past experiences, it is important to remember the specific circumstances under which the event took place, that is, event-specific episodic information. According to hierarchical theories of autobiographical memory, (e.g., the Selfmemory System; Conway \& Pleydell-Pearce, 2000), such episodic detail is accessed at the lowest, temporally specific, memory level. Defined in this way, the reporting of episodic memories and episodic detail could be conceptualised as involving similar underlying processes. However, episodic memories can vary considerably in terms of the amount of episodic detail embedded within them. For example, a particular temporally specific memory can be low in detail (e.g., "when I went to the beach last Friday"), or, relatively, high (e.g., "when I went to Kaiteriteri beach on Friday with my sister Hannah and my mum and it was sunny and we played Volleyball"). Therefore, tendency to report specific memories is not necessarily an indicator of tendency to report specific memories that are rich in episodic detail.

Supporting this notion is the finding that adults' tendency to report higher numbers of temporally specific memories was not associated with concurrent tendency to report specific memories that are highly detailed (Kyung et al., 2016). In this cross-sectional study, 654 young adults completed the Autobiographical Memory Test (AMT; Williams \& Broadbent, 1986), and self-report measures of psychological functioning. The AMT is a cueing paradigm that is commonly used in research on memory specificity and instructs participants to report 
episodic memories in response to emotionally valanced cue words (typically positive and negative, but some studies also include neutral cues). For each participant, Kyung et al. calculated the number of specific memories reported across all cue words, and the average amount of episodic detail embedded within specific memories. Detail in overgeneral memories was not analysed because of the assumption that it most likely reflects semantic knowledge, rather than event-specific detail. Individuals who reported higher numbers of specific memories on the AMT did not report specific memories that were more detailed than individuals who reported fewer specific memories. The authors concluded that, within the constraints of the AMT measure, reporting of specific memories and reporting of detail within specific events might represent different underlying constructs.

Moreover, Kyung et al. (2016) found that memory specificity and episodic detail related differentially to concurrent depression and rumination levels, albeit weakly, when controlling for the effect of the other. That is, whereas higher specificity was associated with lower levels of concurrent depressive symptoms and ruminative thinking, higher average levels of detail across specific memories were associated with higher levels of concurrent depressive symptoms and ruminative thinking. Interestingly, findings were limited to memories reported in response to cue words that were conceptualised as neutral in valence. When the authors ran the same analyses across all cue words (positive, negative, and neutral), lower specificity predicted higher depression levels $(\beta=-.08, p=.040)$, but not rumination levels. Higher episodic detail, on the other hand, predicted higher levels of rumination $(\beta=$ $.02, p=.010)$, but not depression levels.

In light of these cross-sectional findings for adults, several gaps in the literature may be identified. First, no research has investigated the relationship between episodic specificity, episodic detail and psychopathology in typically developing youth. Anxiety and depression levels increase dramatically during adolescence (Costello et al., 2006), however, and reduced memory specificity represents one form of cognitive vulnerability (e.g., Gutenbrunner et al., 2017; Hamlat et al., 2015). Considering the finding that memory specificity and detail relate differentially to psychopathology in adults (Kyung et al., 2016), it will be important to establish: first, whether these two dimensions of autobiographical memory are related in youth, and second, whether the dimensions' associations with psychological functioning are similar or different. Answering these questions will provide novel insights into the role of low memory specificity in the development of early psychopathology above and beyond other aspects of memory. 
The second gap in the literature is that although longitudinal research is critical for understanding causal relationships, no research has tested these aforementioned associations over time. The third gap is that although theory and supporting empirical evidence suggest that the reverse order has some support - namely that poor psychological functioning predicts reduced memory specificity (e.g., Rawal \& Rice, 2012a; Williams et al., 2007)—no research has investigated whether this is also the case for individual variability in memory detail. Lastly, in light of the finding that rumination can exacerbate the effect of memory specificity on psychopathology in community youth (e.g., Hamlat et al., 2015), it may prove fruitful to also test whether rumination moderates associations between memory detail and prospective psychopathology in this population.

The current study drew on, and extended, Kyung and colleagues' (2016) crosssectional research with adults. Specifically, we investigated associations between memory specificity, episodic detail and various indicators of psychological functioning in adolescents. First, we tested concurrent associations between memory specificity and degree of detail contained within specific memories. In line with Kyung et al.'s findings, we predicted that these memory dimensions would not be associated. Second, we tested the extent to which proportion of specific memories and amount of detail reported in specific memories would predict the development of psychopathology (i.e., depression and anxiety) and rumination across three annual assessment points. In light of Kyung et al.'s findings, we expected higher memory specificity to predict decreases in symptoms of depression and rumination, but not anxiety, and, conversely, we expected higher memory detail to predict increases in depression and rumination, but not anxiety. Moreover, in light of our previous research findings (BLINDED FOR REVIEW), we hypothesized that associations between memory indices and depression and anxiety would be stronger for youth at risk for psychopathology, namely those who engage in higher levels of rumination over time. We tested this idea by carrying out additional analyses where we included longitudinal rumination tendency as a moderator of associations.

Third, we also tested whether indices of psychological functioning (i.e., depression, anxiety, and rumination), in turn, would predict change in the two memory dimensions (i.e., memory specificity and episodic detail) across the three time points. In addition, we investigated differences in patterns of association between depression and anxiety with memory indices as a function of longitudinal rumination tendency. Due to the exploratory nature of these analyses, we did not form a priori hypotheses. Lastly, our own and other past research suggests that associations between memory specificity and psychopathology may 
initially be limited to memories elicited in response to negative cue words in young people, and generalise to other cue valences when psychopathology increases (e.g., Kuyken \& Dalgleish, 2011; Rawal \& Rice, 2012b). In light of this notion, across all longitudinal analyses, we expected stronger associations between memory indices and psychopathology for memories reported in response to negative cue words, as opposed to positive cue words.

\section{Method}

\section{Participants}

Ninety-six New Zealand secondary school students (34 females, 62 males) participated in the current study. Participants' mean age was 13.6 years $(S D=1.0)$ at the first of the three annual waves. At each time point, parental consent and student assent was obtained, allowing for withdrawal from the study. The participants in the present study represent a subsample drawn from a larger longitudinal dataset $(N=269$; see BLINDED FOR REVIEW, for more information), and were selected on the basis of their longitudinal rumination tendencies (see analytic strategy section). Selected participants identified as New Zealander or European New Zealander (86.3\%), Asian (6.3\%), Maori (3.2\%), Pacific Islander $(2.1 \%)$ or Other $(2.2 \%)$. The majority of participants were of middle socio-economic background. This research was approved by the School of Psychology Human Ethics Committee, under delegated authority to the Victoria University of Wellington's Ethics Committee.

\section{Measures}

Depression. The 12-item self-report short form of the Children's Depression Inventory-2 (CDI-2:SR[S]; Kovacs, 1985, 1992) was administered. For each item, participants are presented with three sentences and asked to endorse the option that best describes how they were feeling over the past 2 weeks (e.g., "I like myself", "I do not like myself', "I hate myself'). Items are then scored on a 3 -point scale ( $0=$ no symptoms, $1=$ mild symptoms, 2 = severe symptoms) and responses summed to form total scores that range from 0 to 24 . Cronbach's alpha ranged from .75 to .82 .

Anxiety. Participants completed the self-report Revised Children's Manifest Anxiety Scale - 2 Short Form (RCMAS-2; Reynolds \& Richmond, 2008). The 10-item scale assesses different aspects of anxiety: social concerns (e.g., "I feel someone will tell me I do things the wrong way”), physiological anxiety (e.g., "Often I feel sick in my stomach”), and worry (e.g., "I often worry about something bad happening to me"). Participants indicate whether each statement is consistent with their experience on a 2-point scale $(0=N o ; 1=Y e s)$, and 
responses are summed to form total anxiety scores that range from 0 to 10 . Cronbach's alphas were good across the three waves $(\alpha \mathrm{s}=.78$ to .80$)$.

Rumination. A shortened 6-item version of the 13-item Ruminative Response subscale of the Children's Response Styles Questionnaire (CRSQ; Abela et al., 2002) was administered. Participants indicate how often they engage in ruminative thinking when they are sad (e.g., "When I am sad, I think about how angry I am with myself') on a 4-point scale $(1=$ almost never; $2=$ sometimes; $3=$ often; $4=$ almost always $)$. The scale as it was used in the current study consisted primarily of items tapping into the brooding dimension of rumination, which has been defined as a "passive comparison of one's current situation with some unachieved standard" (Treynor et al., 2003). Average scores across the six items were computed and used for analyses, with values ranging from 1 to 4 . The measure demonstrated good internal reliability ( $\alpha \mathrm{s}=.83$ to .88$)$.

Autobiographical Memory. Autobiographical memories were elicited using an adapted version of the written version of the Minimal Instructions Autobiographical Memory Test (Mi-AMT; Debeer et al., 2009). The Mi-AMT has been found to be more sensitive to memory specificity in non-clinical populations than the original AMT used by Williams and Broadbent (1986). Participants were presented with 10 cue words (happy, sad, lucky, angry, proud, lonely, excited, guilty, relaxed, scared) that alternated between positive and negative valence and were selected from cue word lists used by previous research with youth of comparable age (e.g., Hipwell et al., 2011). After piloting, we made minor alternations to the Mi-AMT by providing more specific instructions. Unlike the original Mi-AMT, we asked participants to think of and write down a specific memory triggered by each cue, and provided examples of correct and incorrect responses. However, in line with the Mi-AMT, and unlike the original AMT, participants did not complete practice items. Participants were told not to use a particular memory more than once, and were given one minute to provide a written response to each cue.

Memory specificity coding and reliability. Responses on the Mi-AMT were coded using pre-established criteria (e.g., Heron et al., 2012). Responses that referred to single events that did not last longer than one day were coded as specific (e.g., "getting my new phone yesterday"); responses that referred to a category of similar events as categoric (e.g., "every time I go to the beach"); and responses that referred to an event that lasted longer than one day as extended (e.g., "when we went to Australia"). In line with previous research, other types of responses were coded as errors and not analysed further. Inter-rater reliability across two trained raters was acceptable $(\kappa=.77)$. Three indices of memory specificity were 
calculated for analyses as proportions of non-missing responses (Griffith et al., 2012): proportions of specific memories, and proportions of specific memories for positive and negative cue words separately.

Episodic detail coding and reliability. The current research adopted the scoring procedure used by Kyung et al. (2016), where amount of memory detail is conceptualised as the sum of unique information units embedded within responses on the AMT that are coded as specific memories. Units of detail can refer to time (when did the event(s) happen), place (where did the event(s) happen), people (who was involved), objects/events (what was involved), actions (what happened), manner (how did the event(s)/action(s) happen), qualifiers (elaborations on other unit details), quotations/thoughts (what was said or thought), causes/intentions (causes or explanations of event(s)/action(s), or emotional responses (reference to emotions). For example, the response "last Sunday (Time), when I was at the park (Place) with my brother (People) to play (Action) with a ball (Object)" would be scored as 5 unit details. For each participant, 3 memory indices were computed: average number of unique details among total number of specific memories reported by the participant, and separate averages for responses reported in response to positive and negative words on the AMT. The first author coded all memories, and a secondary rater coded $25 \%$ of the data; the obtained single measures intraclass correlation coefficient (ICC) was .86 and indicates a high level of agreement.

\section{Procedure}

Data were collected during school time in groups of approximately 10 students. Before assessment, students were informed about confidentiality and told that responses would only be traced back to participants if they created a cause for concern for a person's safety. Upon informed consent, participants completed the Mi-AMT, followed by the selfreport questionnaires. Debriefing included information about the purpose of the study and the opportunity to ask questions. Data collection sessions were of approximately 45 minutes' duration and carried out by trained postgraduate students and research fellows.

\section{Missing Data}

In the larger dataset from which the current subsample was selected, data were missing completely at random, Little's $\operatorname{MCAR} \chi^{2}(2716, N=269)=2711.78, p=.520$. Total percentage of item-level data was $1.90 \%$, and data were imputed using the expectationmaximization (EM) algorithm in SPSS to effect maximum statistical power (see BLINDED FOR REVIEW, for more information). The pattern of missingness in the current subset of 
data was consistent with that of the larger dataset, Little's $\operatorname{MCAR} \chi^{2}(709, N=96)=716.24, p$ $=.417$.

\section{Analytic Strategy}

Participants in the current sample were selected on the basis of their rumination trajectories that we modelled in previous research. Two subgroups were identified that demonstrated either low or medium/high longitudinal levels of rumination across three annual assessment points using a latent class growth analysis (see BLINDED FOR REVIEW, for more information). For the current study, we selected even numbers of participants from these two subgroups $(N=48)$ in order to maximally contrast low vs. moderate/high levels.

Across the three time points, a small number of participants did not provide any specific memories, or only reported specific memories to one type of cue valence. Although these values are absent, they do not represent missing data and therefore cannot be imputed; that is, instead of reporting specific memories in response to all ten cue words, some participants reported some memories that were non-specific. Depending on memory index (specific memories to positive cues, negative cues, or combined), $2.1 \%$ to $8.3 \%$ of data was absent at each time point; patterns of absence were determined to occur completely at random (Little's MCAR tests were non-significant). As all participants reported specific memories for at least one time point, we adopted the method of pairwise deletion, which maximises data available by an analysis-by-analysis basis. This form of available-case analysis minimises the loss in power that occurs in list-wise deletion, where all participants with one or more missing values across time points are excluded from all analyses.

Preliminary analyses were run first to obtain descriptive statistics. Correlational analyses were run to test the association between young people's tendency to report specific memories and the amount of contextual detail they report within specific memories. For each time point, and in line with Kyung et al. (2016), we tested the association between proportion of reported specific memories and average amount of detail across specific memories.

To test our second hypothesis that autobiographical memory specificity and amount of detail would relate differentially to indices of psychological functioning across the entire sample, we ran longitudinal autoregressive cross-lagged models. All structural equation models were tested using AMOS (Arbuckle, 2014). The two memory dimensions were included as predictors of depression, anxiety, and rumination, controlling for the effects of age and gender. Average word count of memories was included as a covariate to ensure that associations between memory detail and outcome measures were not due to individual 
variability in length of responses. Predictors were allowed to covary with each other, and so were outcome measures. Due to the sample size, separate analyses were carried out for the first time lag (predicting change in outcome measures at Time 2 as a function of predictors at Time 1) and the second time lag (predicting change in outcome measures at Time 3 as a function of predictors at Time 2). Hypotheses were tested using bias-corrected $95 \%$ bootstrapped confidence intervals (CI) (2000 samples). Analyses relating to our third research question - whether psychological functioning differentially predicts memory specificity and detail - followed the same statistical approach, but psychological indices were specified as predictors of memory specificity and detail in all models.

To test whether associations between memory dimensions and psychological functioning varied across young people with different rumination tendencies, multi-group analyses were carried out for each of the models described above. Here, main effect coefficients were computed separately for the low and medium/high rumination trajectory subgroups. If a coefficient was found to be statistically significant in either trajectory group, follow-up chi-square difference tests were carried out. That is, a group difference was considered significant only if the chi-square value significantly increased as a function of constraining the pathway of interest to equality across trajectory subgroups (indicating worse model fit).

\section{Results}

\section{Descriptive Statistics}

Study variables' means and standard deviations are reported separately for youth in the low and medium/high rumination growth groups in Table 7. As expected, means for depression, rumination, and anxiety were consistently higher for the medium/high subgroup compared to the low subgroup across the three waves. Multivariate analyses of variance showed that differences were statistically significant at the $p<.001$ level. In terms of memory characteristics, proportions of specific memories reported did not differ significantly across the two rumination subgroups at any time point ( $p s=.074$ to .629 ). Two significant differences between the groups was found for amount of detail reported; at Time 1, youth in the low rumination growth group reported significantly greater amount of detail to both positive and negative cue words, $p=.035$ and $p=.010$, respectively. 
Table 7

Descriptive Statistics of the Study Variables for Low and Medium/High Rumination Growth Subgroups

\begin{tabular}{|c|c|c|c|c|c|c|}
\hline & \multicolumn{2}{|c|}{ Wave 1} & \multicolumn{2}{|c|}{ Wave 2} & \multicolumn{2}{|c|}{ Wave 3} \\
\hline & Low & Med/High & Low & Med/High & Low & Med/High \\
\hline Prop. SPMs & $.65(.26)$ & $.62(.24)$ & $.72(.19)$ & $.65(.23)$ & $.74(.16)$ & $.67(.23)$ \\
\hline Neg. cues & $.59(.30)$ & $.56(.28)$ & $.66(.26)$ & $.60(.25)$ & $.66(.21)$ & $.57(.28)$ \\
\hline Pos. cues & $.71(.28)$ & $.68(.27)$ & $.77(.23)$ & $.70(.29)$ & $.82(.17)$ & $.76(.24)$ \\
\hline Average detail & $4.39(.79)$ & $3.88(.92)$ & $4.33(.97)$ & $4.24(.88)$ & $4.50(1.07)$ & $4.53(.98)$ \\
\hline Neg. cues & $4.46(.94)$ & $3.98(1.19)$ & $4.34(1.19)$ & $4.24(1.04)$ & $4.61(1.34)$ & $4.56(1.22)$ \\
\hline Pos. cues & $4.32(.98)$ & $3.81(.92)$ & $4.35(1.09)$ & $3.88(1.50)$ & $4.42(1.17)$ & $4.52(1.07)$ \\
\hline CDI-2 & $2.54(2.11)$ & $6.06(3.24)$ & $2.46(2.01)$ & $7.07(4.00)$ & $3.73(2.80)$ & $7.94(3.60)$ \\
\hline CRSQ & $1.38(0.30)$ & $2.46(2.59)$ & $1.39(0.29)$ & $2.78(0.59)$ & $1.51(0.32)$ & $2.77(0.45)$ \\
\hline RCMAS-2 & $1.85(2.07)$ & $4.56(2.59)$ & $2.04(2.15)$ & $4.93(2.47)$ & $2.33(2.18)$ & $5.37(2.26)$ \\
\hline
\end{tabular}

Note. Prop. SPMs = Proportion of specific autobiographical memories; CDI-2 = Children's Depression Inventory 2; CRSQ = Children's Response Styles Questionnaire; RCMSA-2 = Revised Children's Manifest Anxiety Scale.

Correlations among study variables and memory dimensions are reported in Table 8 . In terms of proportions of specific memories and amount of detail contained in specific memories, five significant correlations were found. Lower proportions of specific memories at Time 2 were associated with higher levels of anxiety at Time 1 and Time 2, and higher levels of depression at Time 1. Lower specificity at Time 3 was associated with higher depression at Time 2. Lower levels of detail in specific memories at Time 1 were associated with higher levels of rumination at Time 2 . However, these findings were significant at the $p$ $<.05$ level and should be interpreted with caution considering the number of comparisons carried out ( 4 out of 81 correlations approximates a probability level of .05 , so some of these significant correlations may have occurred by chance). Average memory word count was included in the correlation analysis to ascertain whether indices of psychological functioning were associated with tendency to report short or long memories independently of detail; no significant correlations were found. 
Table 8

Pearson Correlations between Memory Dimensions and Other Key Variables

\begin{tabular}{|c|c|c|c|c|c|c|c|c|c|}
\hline & \multicolumn{3}{|c|}{ Average Detail } & \multicolumn{3}{|c|}{ Prop. SPMs } & \multicolumn{3}{|c|}{ Average WC } \\
\hline & $\mathrm{T} 1$ & $\mathrm{~T} 2$ & T3 & $\mathrm{T} 1$ & $\mathrm{~T} 2$ & $\mathrm{~T} 3$ & $\mathrm{~T} 1$ & $\mathrm{~T} 2$ & T3 \\
\hline CDI-2 T1 & -.18 & -.04 & .02 & .01 & $-.21 *$ & -.19 & -.10 & -.06 & -.10 \\
\hline CDI-2 T2 & -.18 & -.08 & -.17 & -.04 & -.20 & $-.25^{*}$ & -.09 & -.03 & -.20 \\
\hline CDI-2 T3 & -.07 & .09 & -.05 & .06 & -.09 & -.08 & .04 & .08 & -.05 \\
\hline CRSQ T1 & -.14 & .05 & .03 & -.07 & -.18 & -.15 & .02 & .07 & .09 \\
\hline CRSQ T2 & $-.22 *$ & .09 & -.02 & -.03 & -.18 & -.11 & -.03 & .16 & .03 \\
\hline CRSQ T3 & -.15 & -.02 & -.05 & -.001 & -.14 & -.15 & .001 & .07 & .04 \\
\hline RCMAS-2 T1 & -.08 & .04 & .15 & -.03 & $-.23 *$ & -.14 & .01 & .04 & .09 \\
\hline RCMAS-2 T2 & -.14 & .12 & .02 & -.13 & $-.21 *$ & -.19 & .02 & .15 & .08 \\
\hline RCMAS-2 T3 & -.12 & .04 & .02 & .05 & -.07 & -.07 & .04 & .10 & .06 \\
\hline
\end{tabular}

Note. CDI-2 = Children's Depression Inventory 2; CRSQ = Children's Response Styles Questionnaire; RCMSA-2 = Revised Children's Manifest Anxiety Scale; T1 = Time 1; T2 = Time 2; T3 =Time 3; Prop. SPMs = Proportion of Specific Memories; WC = Word Count. $* p<.05$.

To test our first hypothesis that proportions of specific memories and units of detail averaged across specific memories would not be associated concurrently, correlation analyses were carried out for each time point (Table 9). The two memory dimensions were not significantly correlated at any time point, which supports our hypothesis and is consistent with previous research (Kyung et al., 2016). Additional correlation analyses showed that average memory word count was significantly correlated with memory detail at each time point ( $r s=.71$ to .79 ), but not with proportion of specific memories. These findings are consistent with previous research (Kyung et al., 2016) and highlight the importance of including average memory word count in all structural equation models as a covariate. 
Table 9

Correlations between Memory Dimensions

\begin{tabular}{lccc}
\hline & \multicolumn{3}{c}{ Average Detail } \\
\cline { 2 - 4 } & T1 & T2 & T3 \\
\hline Prop. SPMs T1 & .03 & .03 & -.10 \\
Prop. SPMs T2 & -.06 & .11 & -.04 \\
Prop. SPMs T3 & .11 & .13 & .10 \\
\hline Note. T1 = Time 1; T2 = Time 2; T3 =Time 3; \\
Prop. SPMs = Proportion of Specific Memories.
\end{tabular}

\section{Memory Specificity and Detail as Predictors of Psychological Functioning}

To test our second hypothesis that memory specificity and detail within specific memories would differentially predict psychological functioning (depression, rumination, and anxiety) across the entire sample, longitudinal autoregressive cross-lagged models were run. All models were run three times: 1) using memories reported across all cue words, 2) using memories reported to positive cue words, and 3) using memories reported to negative cue words. As models were run separately for the two time lags, six cross-lag effect tests were carried out per analysis (memory specificity and detail predicting change in depression, rumination, and anxiety). Age, gender and average word count were included as covariates in all analyses. No significant effects between memory specificity or detail and indices of psychological functioning were found across either time lag. Our second hypothesis that memory indices would predict psychological functioning differentially was therefore unsupported.

Next, multi-group analyses were run to test whether memory specificity and detail would predict indices of psychological functioning differentially across youth with either low or medium/high longitudinal rumination trajectories. That is, the moderating role of rumination trajectory on associations between the two memory dimensions and anxiety and depression was tested. No significant group differences were found across the two time lags. These findings are inconsistent with our hypothesis that rumination would moderate the effects of memory specificity and episodic detail on psychopathology.

\section{Psychological Functioning as a Predictor of Memory Specificity and Detail}

Next, to test whether anxiety, depression, and rumination levels would predict change in the two memory dimensions over time, we ran structural equation models that specified the three indices of psychological functioning as predictors of memory specificity and detail. As 
these analyses were exploratory, we did not form specific hypotheses about associations across the entire sample, or possible differences across rumination trajectory groups. We found one significant association across the entire sample, out of 36 comparisons; higher levels of depression at Time 2 predicted lower proportions of specific memories at Time $3, \beta$ $=-.35, p=.036, \mathrm{BC} 95 \% \mathrm{CI}[-.789,-.021]$.

To test whether psychological functioning would predict change in the two memory dimensions differently across rumination trajectory groups, we examined relevant pathway coefficients in the previously described multi-group models. Two out of the 36 group comparisons were significant. For each, chi-square difference tests were significant, indicating significantly worse model fit when the pathway of interest was constrained to equality across the two rumination groups. First, higher levels of anxiety at Time 1 predicted significantly lower proportions of specific memories at Time 2 in the medium/high rumination group, $\beta=-.41, p=.017, \mathrm{BC} 95 \% \mathrm{CI}[-.772,-.080]$, but not the low rumination group, $\beta=.20, p=.391$, BC 95\% CI [-.227, .564]. Second, higher anxiety levels at Time 2 predicted more detailed specific memories in response to negative cue words in the low rumination trajectory group, $\beta=.39, p=.018, \mathrm{BC} 95 \% \mathrm{CI}[.107, .742]$. For the medium/high trajectory group a trend in the opposite direction was found; increased levels of anxiety predicted lower levels of detail within specific memories reported to negative cue words, $\beta=$ $-.44, p=.064, \mathrm{BC} 95 \%$ CI $[-.779, .019]$.

In summary, with regard to our third research question-whether psychological functioning would predict change in memory specificity and detail-higher depression levels predicted lower memory specificity across the entire sample from Time 1 to Time 2. Moreover, higher levels of anxiety interacted with rumination trajectory to predict memory specificity and detail over time. All three effects were found across single, and different, time lags, however, indicating that psychopathology did not consistently predict episodic specificity and detail.

\section{Discussion}

The current study was conducted in order to achieve three major aims: first, to test associations between autobiographical memory specificity and episodic detail using the MiAMT in community adolescents; second, to examine similarities and differences in associations between these memory dimensions and psychological functioning longitudinally across three time points; and third, to investigate the moderating role of rumination tendencies on longitudinal associations between memory characteristics and psychopathology 
(depression and anxiety). With respect to the first aim, and supporting our first hypothesis, reporting of more specific memories on the Mi-AMT was not associated with higher levels of episodic detail in specific memories. With respect to the second aim, and not supporting our hypothesis, memory specificity and episodic detail did not predict change in psychopathology. With respect to our research question of whether psychopathology predicts memory indices, for which we did not form specific hypotheses, we found transient effects of depression on memory specificity, as well as effects of anxiety, in interaction with rumination growth, on memory specificity and detail. None of these longitudinal findings were consistent across the two time lags, however. In summary, we found limited evidence for relationships among memory specificity, memory detail, and psychopathology in this community-based adolescent sample.

\section{Association between Episodic Specificity and Detail}

Youth who reported more specific memories on the Mi-AMT did not report more episodic detail in specific memories within or across any of the three time points. Therefore, although specific memory retrieval may entail activation of event-specific information, as suggested by hierarchical models of autobiographical memory (e.g., SMS; Conway \& Pleydell-Pearce, 2000), the number of specific memories young people report are not an indication of degree of detailed elaboration of those memories. This finding is consistent with the only other study that investigated this relationship in an adult sample (Kyung et al., 2016). Similarly, other research with adults did not find an association between episodic specificity and detail, although memory indices were operationalised differently (Habermas \& Diel, 2013; Lemogne et al., 2006). Moreover, common but also distinct neural processes are activated during the retrieval and elaboration of specific events in fMRI research with adults (e.g., Addis, Wong, \& Schacter, 2007). As suggested by Kyung and colleagues, these findings lend weight to the argument that memory specificity and detail may represent independent constructs. In the current study, we present novel evidence that reporting of specific memories and reporting of episodic detail, when elicited by the cued and timeconstrained Mi-AMT, are also not correlated in youth.

\section{Longitudinal Associations between Memory Indices and Psychological Functioning}

Neither the number of specific memories that the young people reported nor the detail contained in those memories consistently predicted longitudinal change in ill-being. These findings differ to those described by Kyung et al. (2016) who found associations between fewer specific memories and higher levels of depression and rumination in adults. In contrast, they found that higher levels of detail in specific memories was associated with higher levels 
of rumination and depression. Substantial differences in populations under investigation and methodologies adopted arguably make comparisons tenuous between their and our findings, however. First, we tested associations longitudinally, while controlling for the effect of known covariates on the outcome measures. Kyung and colleagues performed a crosssectional study, and did not control for the effects of other established covariates on their outcome measures. Moreover, their findings were limited to neutral cue words, which we, in line with other studies in this area of research, did not assess.

Second, our participants were adolescents, whereas Kyung and colleagues' study was based on adults. Research has identified mid-to-late adolescence and young adulthood as critical times for increases in psychopathology (e.g., Hankin et al., 1998), which raises the possibility that psychopathology levels in the current sample were, on average, lower than those in Kyung's young adult sample. Perhaps memory specificity and episodic detail elicited on the AMT represent a vulnerability at increased levels of psychological distress, as has been discussed by previous research with respect to memory specificity (Crane et al., 2016). Lastly, it is also important to note that despite the large size of Kyung et al.'s sample $(N=$ 654), significant associations were small in absolute size. Thus, discrepancies in findings may also be due to differences in statistical power and associated constraints relating to detection of effects.

Despite the predominant pattern of null findings, we noted one significant transient effect. Higher levels of depression predicted fewer specific memories across the second time lag. While past research has focused on reduced memory specificity as a vulnerability marker of depression in youth (e.g., Hipwell et al., 2011), few studies have investigated the reverse relationship. That is, whether depression levels predict change in memory specificity. Moreover, past studies have largely recruited at-risk youth with current or past depression (e.g., Rawal \& Rice, 2012b), which makes it difficult to disentangle early temporal patterns of association between low mood and memory specificity. Our finding provides preliminary evidence that under some circumstances, reduced memory may be secondary to low mood during adolescence. Although we did not assess the function of low memory specificity, in our previous research (BLINDED FOR REVIEW) we found that cognitive avoidance predicted OGM in youth. Perhaps young people in this sample recalled fewer specific memories in the context of low mood to avoid activation of unpleasant memory-related affect (Williams, 1996; Williams et al., 2007).

\section{Rumination as a Moderator of Associations}


No consistent moderating effects of rumination on associations between memory constructs and psychopathology were found. Two significant findings emerged, but they were unstable across time. First, higher levels of anxiety predicted fewer specific memories for youth who reported higher rumination levels across time. Perhaps young people who attempt to regulate anxious distress by engaging in rumination are more prone to developing an equally non-specific style of remembering their past to manage distress. Indeed, rumination and non-specific remembering have been conceptualised as similarly abstract ways of processing information (Watkins \& Moulds, 2005), and rumination represents an established underlying mechanism of reduced memory specificity in people who experience emotional distress (Raes, Watkins, Williams, \& Hermans, 2008; Williams et al., 2007). Moreover, rumination has been found to moderate the association between anxiety and memory specificity in adults (Hallford \& Mellor, 2017).

Highly anxious youth reported more episodic detail in specific event memories, but only in response to negative cue words, and only when they engaged in low levels of rumination over time. For anxious youth who ruminated more, a trend in the opposite direction was found; they reported less episodic detail in specific memories reported to negative cues. Perhaps anxious youth who ruminate more are less likely to report eventspecific detail because processing of negative information in a general and abstract form is more habitual. In low-ruminating youth, on the other hand, higher levels of detail may represent more concrete information processing, which entails reflection on details of past experiences to process and manage current distress (Watkins, Baeyens, \& Read, 2009). Our data, unfortunately, do not allow us to address these possibilities; nonetheless, the current findings may be preliminary evidence that rumination can play a role in how anxious youth learn to remember their past.

\section{Methodological Considerations, Limitations and Conclusions}

We note that our measures of memory specificity and episodic detail represent performance on the Mi-AMT, a cued and time-constrained test. This measure was not designed to quantify memory retrieval ability or access. That is, young people in our study were not instructed to retrieve specific memories with as much detail as possible. Episodic detail in memories as assessed by the Mi-AMT may not indicate the amount of detail an individual is able to recall, or access, but rather reveal naturally occurring individual variability in the style of reporting such information on the Mi-AMT. In other research with youth, higher levels of episodic detail in turning point memory narratives — with instructions to retrieve as much detail as possible_ - predicted higher levels of depression over time 
(Salmon et al., under review). When interpreting associations between autobiographical remembering and psychological functioning, therefore, it is important to do so in the context of the methodology used (e.g., time constraints, specific instructions, cue valence, and type of memory elicited).

We measured detail only in temporally specific memories - in line with Kyung et al.'s (2016) research - to capture event-specific information, rather than semantic knowledge.

That is, theory suggests that although non-specific memories may contain semantic information (e.g., general similarities of repeated events), a defining feature of episodic recollection is remembering, or re-experiencing, the context in which a specific event was encoded (i.e., sensory-perceptual information; Lemogne et al., 2006; Tulving, 2001). Interestingly, researchers have pointed out that reporting of specific memories on cued memory tasks such as the AMT may not necessarily entail remembering of contextual detail, but rather reflect semantic knowledge (e.g., Griffith et al., 2012). In other words, a person may know where they were on a particular day, rather than remember the context. We, therefore, cannot assume that all contextual information in our participants' memories was truly remembered episodic detail. Indeed, the memory reports were, on average, relatively brief, and information units in them often referred to time and space. For a more comprehensive understanding of longitudinal associations between psychological functioning and different levels of memory (semantic and episodic) in youth, the field would arguably benefit by using additional memory retrieval tools. For example, measures that are designed to capture multiple dimensions of autobiographical memory, including subjective sense of remembering (e.g., the TEMPau task; Piolino, Desgranges, \& Eustache, 2009).

The current study has several limitations. None of our findings were consistent across both time lags. This transiency of effects may indicate that additional moderating, or mediating, variables blunted the strength of associations. For example, a measure of stress would have been valuable in light of findings that implicate stress in the relationship between memory specificity and depression in youth (Hamlat et al., 2015; Stange et al., 2013). Another limitation of the current study was statistical power. The large number of models run to test the current hypotheses, in combination with our relatively small sample size, warrants caution when interpreting findings as Type 1 error rate may have been inflated.

In summary, our findings provide novel evidence that adolescents who report more specific memories on the Mi-AMT do not also report more episodic detail in their memories. In addition, whereas the finding that memory specificity did not predict psychopathology in community youth is consistent with past studies (e.g., Crane et al., 2016), we extend this 
research by showing that episodic detail on the Mi-AMT, likewise, does not shed light on psychological functioning in this population. An important implication is that inferences about young people's tendency to report detailed episodic memories cannot be made on the basis of their performance on this particular measure. It is possible that the two aspects of memory represent independent constructs, although findings are likely moderated by population characteristics and measurement constraints. More research is needed to draw stronger conclusions about the relationship between the two facets of autobiographical memory, and how they relate to the initial emergence of psychological difficulties in adolescence. 


\section{Chapter 5 \\ General Discussion}

The overarching aim of this body of research was to conduct a comprehensive investigation of associations between OGM and psychological functioning across the course of adolescence. Specifically, the three empirical studies in this thesis aimed to: 1) test the influence of OGM on development of early psychopathological symptoms in community youth; 2) establish whether the CaR-FA-X model explains significant variability in OGM in this population; and 3) elucidate associations between OGM, episodic detail, and psychological functioning. In this general discussion I provide an overview of the main findings and discuss how they inform our understanding of OGM in young people. I also make recommendations for future research and outline the current findings' clinical implications. As in previous chapters of this thesis, the abbreviation $O G M$ broadly refers to retrieval of fewer specific memories and retrieval of more overgeneral memories (categoric and/or extended).

\section{Longitudinal Associations between OGM and Depression}

Given the proposed role of OGM in the development and maintenance of major depression, investigation of OGM's early influence on psychological functioning, prior to increases in psychopathology during adolescence, is critical. In Study 1, we, therefore, across three annual time points. In a large sample of community adolescents, OGM did not predict change in depressive symptomatology. This result supports previous findings for community youth across two time points (Crane et al., 2016). Two other longitudinal community youth studies demonstrated an association between OGM and depression, but reported effects that were a function of higher levels of emotional abuse (Stange et al., 2013) and higher levels of rumination and life stress (Hamlat et al., 2015). Collectively, findings for community adolescents suggest that OGM may only represent a vulnerability marker in youth who are already at risk for adversity (Crane et al., 2016).

In Study 1, we sought to extend research on possible moderating variables of associations between OGM and depression by operationalizing "risk" in terms of young people's longitudinal patterns of rumination. By doing so, we aimed to identify youth for whom this cognitive style of processing emotions is relatively habitual and enduring. Patterns of rumination growth across time did not moderate associations between OGM and depression, which is inconsistent with Hamlat et al.'s (2015) findings. Notably, in their study the interactive effect between OGM and rumination was a function of stress levels, which we 
did not control for. Our findings are important because they indicate that rumination alone may not be sufficient to exacerbate the effect of OGM on depression in community youth. Rather, the relationship between OGM and emerging depression may be qualified by complex interactions among multiple risk factors in community youth, including stress. This notion is in line with models of depression that hypothesize that cognitive vulnerabilities, such as rumination, interact with life stressors to promote the onset of depressive symptoms in adolescence (e.g., Response Styles Theory; Nolen-Hoeksema \& Girgus, 1994). Our and other research point toward the possibility that the role of OGM, as a cognitive vulnerability of depression, may also be a function of additional risk factors in community youth.

In Study 3, we broadened the focus on the relationship between OGM and depression by testing the reverse temporal relationship. Overall, patterns of association were nonsignificant across three annual time points. These findings are novel because longitudinal studies typically test the effect of OGM on psychopathology in community youth, and not vice versa. One transient effect of depression on OGM emerged. Specifically, we found that higher depression levels predicted reporting of fewer specific memories across one of the two time lags. As we controlled for baseline levels of OGM and rumination-a covariate of both OGM and childhood depression (Hamlat et al., 2015; Kuyken et al., 2006) — it is unlikely that the relationship we found between depression and OGM is a proxy for an effect of rumination on OGM. Indeed, additional analyses highlighted that rumination growth did not moderate the association between depression and OGM. Perhaps OGM emerges in response to low mood as a form of emotion regulation (Williams, 1996).

A theoretical implication of findings from Studies 1 and 3, collectively, is that OGM and depression are, to the most part, unrelated in community youth. Contrary to the common assumption that OGM is a precursor of depressive symptoms, our findings suggest that this memory bias does not represent a pre-existing risk factor of depression for typicallydeveloping young people. Perhaps OGM exacerbates depressive symptoms in this population once psychological distress reaches a critical threshold (Rawal \& Rice, 2012b; Sumner et al., 2011). Moreover, in this context, OGM may interact with other risk factors to maintain distress. For example, at high levels of depression, compromised emotion regulation and reduced executive control likely facilitate OGM, which, in turn, impedes adaptive emotional adjustment (Williams et al., 2007). Interestingly, our findings suggest that for community youth, OGM may, to some degree, be a consequence, rather than predictor, of low mood. Notably, this effect was limited to one of two time lags, which could indicate that this 
relationship does not occur in isolation. Future research is needed to replicate this finding and determine if other variables moderate, or mediate, the impact of low mood on OGM.

\section{Longitudinal Associations between OGM and Anxiety}

In Study 1, we further extended the field by demonstrating that OGM also does not explain significant variability in general anxiety levels in community youth. This result is consistent with findings from studies with clinical youth populations (de Decker et al., 2003; Rawal \& Rice, 2012b). Importantly, our rigorous statistical approach allowed us to test these relationships whilst controlling for concurrent and prospective associations among depression, OGM, and anxiety. This finding lends further support to the argument that OGM does not represent an early marker of vulnerability that predicts psychopathology in community adolescents.

Although associations were non-significant across the entire sample, we found some evidence that OGM predicted increases in anxiety in young people who are prone to ruminate. The opposite trend was found for youth who tend not to ruminate. Although no research with youth has tested these relationships, a recent study with adults identified rumination as a mediator of the association between anxiety and OGM (Hallford \& Mellor, 2017). Addressing this research question in youth is important because rises in anxiety typically precede and predict increases in depression in youth (Cole, Peeke, Martin, Truglio, \& Seroczynski, 1998). As depression levels in our study were low compared to other community youth research (e.g., Hamlat et al., 2015), our finding suggests that OGM, in interaction with higher rumination, may influence anxious symptomatology prior to initial increases in depression.

Why does OGM predict poor emotional adjustment in the context of high rumination? Past research has described the two constructs as similarly abstract and mutually reinforcing risk factors for psychopathology (Raes et al., 2006b; Watkins \& Teasdale, 2001, 2004). Specifically, whereas rumination may hijack the hierarchical search for a specific memory, recall of non-specific memories can, in turn, consolidate and increase accessibility of general negative self-beliefs, thus exacerbating ruminative self-focus (Williams, 1996; Williams et al., 2007). It is possible that for youth with long-standing rumination tendencies, OGM emerges as part of a broader host of abstract information processing biases that have negative consequences. In contrast, for adolescents who are not prone to ruminate, OGM may serve a protective function that fosters well-being. Indeed, a recent experimental study demonstrated that children and adolescents who are instructed to recall negative past events in an 
overgeneral manner report higher levels of positive affect than youth who are asked to retrieve specific memories (Bunnell \& Greenhoot, 2018).

The associations between OGM and anxiety across the two rumination subgroups were limited to negative cue words. This finding may be supportive of the idea that OGM is initially used to ward off unpleasant emotional experiences that retrieval of negative event memories can trigger (Hermans et al., 2008b; Williams, 1996; Williams et al., 2007). Over time, and as distress increases, OGM may develop into a more globalized and inflexible style of remembering the past that generalizes to positive cue words (Kuyken \& Dalgleish, 2011). Indeed, differences in associations between indices of psychopathology and OGM to negative and positive cue words may reflect differences in the flexibility with which young people adopt such a memory retrieval style to regulate affect (Hitchcock, 2014). Perhaps emotional distress in this community sample was not sufficiently high for OGM to develop into a response style that extends to all memories.

Findings from Study 3 further demonstrated that higher anxiety and rumination in conjunction may facilitate reporting of fewer specific memories. This result suggests that young people with a tendency to engage in abstract information processing - in the context of anxious distress - may be more likely to develop OGM. Again, the effects were transient, however, likely indicating that associations only emerge under particular circumstances. Regardless, findings from Study 1 and Study 3, in combination, are the first to demonstrate a possible bidirectional association between OGM and anxiety in adolescence, which challenges the predominant belief that the two constructs are unrelated (Williams et al., 2007). Importantly, rumination appears to moderate this relationship.

In summary, the overarching patterns of findings for the entire sample highlight that OGM and anxiety are unrelated in community youth. The finding that rumination exacerbated the effect of OGM on anxious mood, and vice versa, albeit transiently, warrants follow-up research. Indeed, studies on memory biases such as OGM in childhood anxiety are lacking (see Lau \& Waters, 2017, for a review). In light of established discrepancies in how OGM relates to other indices of cognitive functioning across different age groups (Stewart et al., 2017), findings for adults should not simply be generalized to young people.

\section{Testing the CaR-FA-X Model in Community Youth}

If OGM represents a risk factor for future adversity, establishing OGM's etiology during this time will have important implications for understanding the emergence of psychopathology. Accordingly, in Study 2, we tested the predominant theoretical account of 
OGM - the CaR-FA-X model (Williams et al., 2007) — in community youth across four annual time points. Overall, the model had limited utility in this sample of adolescents, which is consistent with other studies that did not find support for the model mechanisms in youth (e.g., Kuyken et al., 2006). The current study extended past research, however, by testing the model in its entirety for the first time in community youth. Importantly, we examined whether the mechanisms represent vulnerabilities for OGM by adopting a longitudinal study design. In light of consistent null effects across three time lags (each of approximately 12-month duration), we conclude that individual variability in OGM during adolescence is better explained by mechanisms other than the three CaR-FA-X components.

Moreover, depression growth did not moderate the degree to which the three model mechanisms predicted OGM in this sample. This analysis had both theoretical and empirical justification. First, the CaR-FA-X model was developed to shed light on OGM in the context of psychopathology such as depression (Williams et al., 2007), and second, the only study to test the model in its entirety in adults found differences in associations across clinically depressed and psychologically healthy participants (Sumner et al., 2014). To our knowledge, the current study provides novel evidence that the CaR-FA-X model does not explain significant variability in OGM as a function of early depression symptom growth in youth. At this point, I would like to reiterate that the CaR-FA-X model was developed to explain OGM in the context of psychopathology. The current conclusions do not refute the model, but rather provide support for the notion that the model mechanisms likely function differently across clinical and non-clinical populations (Sumner, 2012).

Despite the predominant lack of support for the model as a whole, increased levels of self-reported cognitive avoidance predicted OGM across the last time lag, but only in youth who reported higher, and increasing, depression trajectories. Perhaps depression levels need to reach a critical threshold for the functional avoidance mechanism to become activated in youth. Indeed, mean reported depression scores in this group only fell into the elevated range of scores for the CDI-2 across the last two time points. This finding suggests that OGM may, to some degree, be a cognitive avoidance strategy in community youth who are experiencing persistent low mood. In this context, OGM may serve an avoidance function that protects the young person from experiencing unpleasant emotional material that retrieval of specific negative events can trigger (Williams, 1996; Williams et al., 2007).

The association between avoidance and OGM was limited to negative cue words. As discussed earlier, stronger effects for negative cues might indicate that OGM initially manifests as a flexible form of affect regulation that is applied to negative event memories 
(Hermans et al., 2008b). In other words, at early developmental stages of the functional avoidance mechanism, OGM allows youth to selectively avoid re-experiencing of negative emotions (Williams et al., 2007). The ease with which young people can switch between specific and overgeneral recall may become compromised with continued use of OGM and reinforcing avoidance of unpleasant material. Chronic and worsening low mood likely facilitates this process due to increasing emotion regulation needs. In the current study, young people's early symptoms of depression had perhaps not reached high enough levels to trigger the processes whereby OGM is also applied to positive event memories.

Why did we not find support for the capture and rumination and executive control mechanisms? Although we refer to subgroups as low and medium/high, the terminology is relative; overall, levels of depression were low in this sample. The two mechanisms purport that insufficient executive control and rigid focus on negative self-schemas can interfere with the search for a specific memory. Notably, both of these cognitive vulnerabilities are hallmarks of depression, which may render their relative contributions to OGM more likely in depressed populations compared to psychologically healthy people (Crane et al., 2007; Sumner, 2012; Williams et al., 2007). Indeed, higher rumination and reduced executive control, in interaction, predict OGM in youth at risk for depression (Rawal \& Rice, 2012a). Perhaps the executive control and capture and rumination mechanisms are activated when low mood worsens and negative self-schemas become more elaborate and accessible (Williams et al., 2007). Further longitudinal research with adolescents is needed to test this developmental possibility.

In this area of research as a whole, considerable variability in methodologies across studies limits the scope for making comparisons and generalizing findings. Conclusions about the CaR-FA-X model's efficacy in the current research can, therefore, only be made in the context of the measures used, and the degree to which they tap into the mechanisms purported by this theory. For example, the first component of the model refers to two processes, namely the capture of attention by negative self-representations, and subsequent rumination. Whereas past research has tested the capture component, by examining presence of negative self-representations in young people's memories (Valentino, Toth, \& Cicchetti, 2009), for example, we only assessed the rumination aspect. Similarly, the model's executive control component is a complex construct that could be operationalized in a myriad of ways. Lastly, although trauma may play an important role in understanding the development of OGM as a form of functional avoidance (Williams, Stiles, \& Shapiro, 1999), we were unable to control for this possibility in our analyses. Clearly, to arrive at a more definitive evaluation 
of this theory's applicability to understanding OGM in young people, the model needs to be tested in its entirety using a range of measures for each proposed mechanism. Nonetheless, our findings provide compelling preliminary evidence that the CaR-FA-X model, as a whole, has limited utility in community youth.

\section{Episodic Detail: Associations with Memory Specificity and Psychopathology}

The third aim of this thesis was to shed light on associations between OGM and other aspects of autobiographical memory. In Study 3, we therefore tested the relationship between young people's tendency to report specific memories and average amount of episodic detail embedded in specific memories. We found that youth who reported more episodic memories did not report more detailed memories, which is consistent with results reported by the only other study that investigated this question in adults (Kyung et al., 2016). These findings suggest that the two facets of episodic recall, as measured by the AMT, represent different underlying processes. Replication of this finding is needed to draw stronger conclusions about possible implications, however. Moreover, as discussed in Chapter 4, mode of memory elicitation needs to be taken into consideration when interpreting this finding. As young people were not instructed to report as much detail as possible, we can only tentatively conclude that young people's tendency to report specific memories is unrelated to their tendency to report episodic detail on the AMT.

With the aim of gaining a better understanding of similarities and differences in relationships between the two memory dimensions and psychopathology, we also tested associations among episodic detail, depression, and anxiety across three annual assessment points. Importantly, we controlled for the effects of memory specificity and detail on each other and the outcome measures in all analyses. Mirroring findings for OGM, which I reported earlier in this chapter, amount of detail in specific memories was not consistently associated with psychopathology across the entire sample. This finding extends past research by demonstrating that episodic detail in specific memories, akin to OGM, does not shed light on emerging psychopathology in community youth.

Although episodic detail and psychopathology were not associated across the sample as a whole, we found that higher levels of anxiety predicted higher levels of episodic detail in memories to negative cue words, but only for youth with low rumination tendencies and only across a single time lag. For high ruminators, the trend was in the opposite direction. Perhaps reporting of episodic detail serves different emotion regulation functions across the two groups, as discussed more extensively in Chapter 4. The finding that rumination moderated 
these associations lends further support to the notion that this transdiagnotic risk factor may be crucial to understanding relationships between anxious distress and young people's memory. Given the transient nature of the effects, however, conclusions can only be made tentatively. More research on the development of memory biases, alongside other cognitive vulnerabilities, and emerging anxiety during adolescence is necessary.

As discussed with regard to OGM, more robust relationships between episodic detail and psychological functioning may emerge when young people experience other cognitive and emotional difficulties, above and beyond rumination. If the function of reduced memory specificity and its impact on well-being differs across depressed and community samples (Crane et al., 2015), the same might be true for episodic detail. Indeed, a study with adults reported less episodic detail in a group of depressed participants, relative to a control group, but they used the Autobiographical Interview (Söderlund et al., 2014). Future research is needed to establish whether clinically depressed youth report less episodic detail on the AMT than non-depressed young people, and whether amount of episodic detail reported relates differentially with other indices of psychological functioning in this population.

\section{Clinical Implications}

The current findings have several clinical implications. One aim of this body of research was to establish whether OGM, as assessed by the AMT, represents a risk factor for early psychopathology and should be targeted as part of preventative interventions. Overall, findings highlighted that OGM alone may not be a fruitful objective for such programmes in community youth as it did not consistently predict increases in depression and anxiety. Similarly, degree of detail in specific memories did not shed light on emerging psychopathology. Findings from this thesis suggest that associations between autobiographical remembering and psychopathology may, to some degree, be dependent on presence of other psychological vulnerabilities. First, higher levels of rumination exacerbated the effect of OGM on increases in anxiety, and second, higher levels of rumination interacted with higher levels of anxiety to predict increases in OGM and decreases in recall of episodic detail. These findings not only underscore the importance of addressing rumination in interventions (Watkins, 2015), but they also suggest that, for youth who report higher rumination, it may prove fruitful to identify and target memory biases more directly. In this context, OGM may be one manifestation of a host of maladaptive and abstract affect regulation processes. 
Promisingly, a recent review of interventions aimed at reducing autobiographical memory biases such as OGM reports positive findings, although the majority of studies were with adults (Hitchcok et al., 2017). In light of the notion that OGM may develop into a harmful form of avoidance when used rigidly, youth at elevated risk for psychopathology may benefit from interventions that target avoidant tendencies and memory retrieval flexibility. Indeed, in Study 2, avoidant tendencies predicted OGM predominantly in youth with enduring elevated depression symptoms. One programme that is currently under evaluation — memory flexibility training (MemFlex; Hitchcok et al., 2015) — may be particularly beneficial for this population as it addresses a range of cognitive biases. Preliminary evidence suggests that MemFlex can increase memory specificity, and reduce both rumination and cognitive avoidance in depressed adults (Hitchcok et al., 2016). Whether or not a programme such as MemFlex would be beneficial for community youth is yet to be determined, however.

\section{Future Research}

One broader methodological limitation of the current thesis is its overreliance on the AMT. This is not only a caveat of the current body of research, but the OGM literature as a whole (Griffith et al., 2012). The predominant use of this specific test limits generalizability of findings. In other words, we can draw conclusions about relationships between psychopathology and how people remember past events on the Mi-AMT, but we cannot assume that such patterns hold up under different conditions of memory elicitation. To complicate the picture further, studies on OGM vary in terms of AMT test instructions, cue words, and mode of administration they use. For example, the Mi-AMT used in this thesis was developed to increase sensitivity of the measure to OGM in community samples (Debeer et al., 2009), so findings are not directly comparable to research that used other AMT versions or non-AMT measures. For a more comprehensive understanding of memory biases in community youth, future research should adopt a broader range of measures (e.g., turning point narratives; see Salmon et al., under review) and conceptualize memory specificity in more than one way (e.g., episodic specificity/OGM vs. episodic detail). The literature would also benefit from a systematic review of associations between OGM and psychopathology in young people as a function of test characteristics and conceptualization of memory specificity.

Further longitudinal investigations of associations between OGM, episodic detail, and psychopathology (depression and anxiety) during adolescence need to be carried out. Such 
studies should span even longer time periods to identify pathways via which OGM may turn into a marker of vulnerability. Importantly, to delineate possible nuanced changes in associations between autobiographical remembering and emerging psychopathology, research needs to recruit large samples, control for covariates of autobiographical remembering and psychopathology (e.g., stress and rumination), and use rigorous statistical approaches. Moreover, although the current thesis examined associations between OGM and episodic detail, future studies should consider broadening the focus of investigation to include additional features of autobiographical memory. One promising direction for future research was recently highlighted by Vanderveren, Bijttebier and Hermans (2017), who discuss similarities between memory specificity and memory coherence (i.e., memories' internal structure). I agree with the authors' argument that exploration of how the two constructs relate to one another may have important theoretical and clinical implications.

Longitudinal investigations into late adolescence and early adulthood may also aid unpacking of how OGM, as a form of emotion regulation, develops into the inflexible style of remembering that is often found in depressed adults. Indeed, past research has implicated several forms of rigid processing (e.g., poor attentional shifting) in the development of adolescent depression (Stange et al., 2016). It would be interesting to explore relationships between OGM and multiple markers of cognitive inflexibility, above and beyond rumination. Also, different memory retrieval tests could be used to shed light on the role of cognitive flexibility, such as the Alternating Instructions AMT (AMT-AI; Dritschel, Beltsos, \& McClintock, 2014), which instructs participants to report both specific and non-specific memories.

As discussed earlier, to arrive at a more conclusive understanding of the CaR-FA-X model's utility in young people, additional longitudinal studies need to test the model in its entirety, using a range of measures for each component, and across longer time periods. Importantly, this research should be carried out with clinical and non-clinical samples. Although the current findings provide preliminary evidence that the model, as a whole, does not explain OGM in community youth, we cannot draw conclusions about the three mechanisms' predictive utility in the context of adolescent psychopathology. Another avenue for future research with community youth could be identification of alternative developmental processes that may underlie individual variability in OGM, such as those proposed in Valentino's (2011) developmental psychopathology model of OGM. Lastly, research outside the OGM literature highlights that retrieval of nonspecific event representations is not harmful, per se, and may even play an important role in adults' adaptive 
psychological functioning (Waters et al., 2014). It would be interesting to explore the selfreported functions of specific and nonspecific memories on various autobiographical memory measures in adolescence, and their associations with both negative and positive psychological outcomes.

\section{Final Conclusion}

Adolescence is critical for the onset of mood disorders such as major depression, and research on longitudinal predictors of early psychopathological symptoms has important theoretical and clinical implications. The aim of this body of research was to shed light on relationships between OGM and emerging psychopathology in community youth, and results extend the literature in several ways. Overall, patterns of findings across the three studies of this thesis suggest that OGM is not an index of poor psychological functioning in typicallydeveloping youth. This style of remembering did not consistently predict depression or anxiety, nor did the three cognitive vulnerabilities that make up the CaR-FA-X model explain significant change in OGM. Moreover, OGM was not related to reporting of episodic detail in specific memories, and episodic detail, in turn, did not shed light on emerging psychopathology. Although I reported a number of transient associations between biases in autobiographical memory and psychological functioning in each study, these findings were qualified by risk for psychopathology and not consistent across time. In summary, results across the three studies indicate that OGM may only represent a marker of vulnerability in adolescence when it presents alongside other established risk factors. 


\section{References}

Abela, J. R. Z., Brozina, K., \& Haigh, E. P. (2002). An examination of the response styles theory of depression in third-and seventh-grade children: A short-term longitudinal study. Journal of Abnormal Child Psychology, 30, 515-527. doi:10.1023/A:1019873015594

Abela, J. R. Z., Rochon, A., \& Vanderbilt, E. (2000). The Children's Response Style Questionnaire. Unpublished questionnaire.

Addis, D. R., Wong, A. T., \& Schacter, D. L. (2007). Remembering the past and imagining the future: common and distinct neural substrates during event construction and elaboration. Neuropsychologia, 45, 1363-1377. doi:10.1016/j.neuropsychologia.2006.10.016

Addis, D. R., Wong, A. T., \& Schacter, D. L. (2008). Age-related changes in the episodic simulation of future events. Psychological Science, 19, 33-41. doi:10.1111/j.14679280.2008.02043.x

Aldao, A., Nolen-Hoeksema, S., \& Schweizer, S. (2010). Emotion- regulation strategies across psychopathology: A meta-analytic review. Clinical Psychology Review, 30, 217-237. doi:10.1016/j.cpr.2009.11.004

Anderson, V., Anderson, P., Northam, E., Jacobs, R., \& Catroppa, C. (2001). Development of executive functions through late childhood and adolescence in an Australian sample. Developmental Neuropsychology, 20, 385-406. doi:10.1207/S15326942DN2001

Arbuckle, J. L. (2014). Amos 23.0 User's Guide. Chicago: IBM SPSS.

Bluck, S. (2003). Autobiographical memory: Exploring its functions in everyday life. Memory, 11, 113-123. doi:10.1080/741938206

Bluck, S., Alea, N., Habermas, T., \& Rubin, D. C. (2005). A tale of three functions: The selfreported uses of autobiographical memory. Social Cognition, 23, 91-117. doi: 10.1521/soco.23.1.91.59198

Brennen, T., Hasanović, M., Zotović, M., Blix, I., Skar, A. M. S., Prelić, N. K., ... GavrilovJerković, V. (2010). Trauma exposure in childhood impairs the ability to recall specific autobiographical memories in late adolescence. Journal of Traumatic Stress, 23, 240-247. doi:10.1002/jts.20513

Bridgett, D. J., Oddi, K. B., Laake, L. M., Murdock, K. W., \& Bachmann, M. N. (2013). Integrating and differentiating aspects of self-regulation: effortful control, executive functioning, and links to negative affectivity. Emotion, 13, 47-63. doi:10.1037/a0029536 
Brittlebank, A. D., Scott, J., Williams, J. M. G., \& Ferrier, I. N. (1993). Autobiographical memory in depression: State or trait marker? British Journal of Psychiatry, 162, 118121. doi:10.1192/bjp.162.1.118

Bunnell, S., \& Greenhoot, A. (2018). Do overgeneral memories make us feel better? An experimental examination. Memory, 26, 74-88. doi:10.1080/09658211.2017.1323105

Burgess, P. W., \& Shallice, T. (1996). Confabulation and the control of recollection. Memory, 4, 359-411. doi:0.1080/096582196388906

Burke, M., \& Mathews, A. (1992). Autobiographical memory and clinical anxiety. Cognition \& Emotion, 6, 23-35. doi:10.1080/02699939208411056

Capaldi, D. M., \& Rothbart, M. K. (1992). Development and validation of an early adolescent temperament measure. Journal of Early Adolescence, 12, 153-173.

Cicchetti, D., \& Toth, S. L. (1998). The development of depression in children and adolescents. The American Psychologist, 53, 221-241. doi:10.1037/0003066X.53.2.221

Ciesla, J. A., \& Roberts, J. E. (2007). Rumination, negative cognition, and their interactive effects on depressed mood. Emotion, 7, 555-565. doi; 10.1037/1528-3542.7.3.555

Conway, M. A. (2005). Memory and the self. Journal of Memory and Language, 53, 594-628. doi:10.1016/j.jml.2005.08.005

Conway, M. A., \& Pleydell-Pearce, C. W. (2000). The construction of autobiographical memories in the self-memory system. Psychological Review, 107, 261-288. doi:10.1037/0033-295X.107.2.261

Costello, E. J., Foley, D. L., \& Angold, A. (2006). 10-year research update review: the epidemiology of child and adolescent psychiatric disorders: II. Developmental epidemiology. Journal of the American Academy of Child \& Adolescent Psychiatry, 45, 8-25. doi:10.1097/01.chi.0000184929.41423.c0

Costello, E. J., Mustillo, S., Erkanli, A., Keeler, G., \& Angold, A. (2003). Prevalence and development of psychiatric disorders in childhood and adolescence. Archives of General Psychiatry, 60, 837-844. doi: 10.1001/archpsyc.60.8.837

Crane, C., Barnhofer, T., Visser, C., Nightingale, H., \& Williams, J. M. G. (2007). The effects of analytical and experiential rumination on autobiographical memory specificity in individuals with a history of major depression. Behaviour Research and Therapy, 45, 3077-3087. doi:10.1016/j.brat.2007.05.009 
Crane, C., Heron, J., Gunnell, D., Lewis, G., Evans, J., \& Williams, J. M. G. (2016). Adolescent over-general memory, life events and mental health outcomes: findings from a UK cohort study. Memory, 24, 348-368. doi:10.1080/09658211.2015.1008014

Cummings, C. M., Caporino, N. E., \& Kendall, P. C. (2014). Comorbidity of anxiety and depression in children and adolescents: 20 years after. Psychological Bulletin, 140, 816-845. doi:10.1037/a0034733

Dalgleish, T., Rolfe, J., Golden, A., Dunn, B., Barnard, P., \& Watson, D. (2008). Reduced Autobiographical Memory Specificity and Posttraumatic Stress: Exploring the Contributions of Impaired Executive Control and Affect Regulation. Journal of Abnormal Psychology, 117, 236-241. doi:10.1037/0021-843X.117.1.236

Dalgleish, T., \& Werner-Seidler, A. (2014). Disruptions in autobiographical memory processing in depression and the emergence of memory therapeutics. Trends in Cognitive Sciences, 18, 596-604. doi:10.1016/j.tics.2014.06.010

Dalgleish, T., Williams, J. M. G., Golden, A. M. J., Perkins, N., Barrett, L. F., Barnard, P. J., ... \& Watkins, E. (2007). Reduced specificity of autobiographical memory and depression: the role of executive control. Journal of Experimental Psychology: General, 136, 23-42. doi: 10.1037/0096-3445.136.1.23

de Decker, A., Hermans, D., Raes, F., \& Eelen, P. (2003). Autobiographical memory specificity and trauma in inpatient adolescents. Journal of Clinical Child \& Adolescent Psychology, 32, 22-31. doi:10.1207/S15374424JCCP3201_03

Debeer, E., Hermans, D., \& Raes, F. (2009). Associations between components of rumination and autobiographical memory specificity as measured by a Minimal Instructions Autobiographical Memory Test. Memory, 17, 892-903. doi:10.1080/09658210903376243

Dickson, K. S., Ciesla, J. A., \& Reilly, L. C. (2012). Rumination, worry, cognitive avoidance, and behavioural avoidance: Examination of temporal effects. Behavior Therapy, 43, 629-640. doi:10.1016/j.beth.2011.11.002

Dritschel, B., Beltosis, S., \& McClintock, S. (2013). An "alternating instructions" version of the Autobiographical Memory Test for assessing autobiographical memory specificity in non-clinical populations. Memory, 22, 881-889. doi:10.1080/09658211.2013.839710

Duckworth, A.L., \& Steinberg, L. (2015). Unpacking self-control. Child Development Perspective, 9, 32-37. doi:10.1111/cdep.12107 
Ellis, L. K., \& Rothbart, M. K. (2001, April). Revision of the early adolescent temperament questionnaire. Poster session presented at the biennial meeting of the Society for Research in Child Development, Minneapolis, Minnesota.

Ganly, T. J., Salmon, K., \& McDowall, J. (2016). Is remembering less specifically part of an avoidant coping style? Associations between memory specificity, avoidant coping, and stress. Cognition and Emotion. Advance online publication. doi: $10.1080 / 02699931.2016 .1227304$

Glynn, R., Salmon, K., \& Jose, P. (2016). The influence of reporting mode on children's cued personal memories. Memory, 24, 1224-1230. doi:10.1080/09658211.2015.1088034

Griffith, J. W., Sumner, J. A., Debeer, E., Raes, F., Hermans, D., Mineka, S., ... \& Craske, M. G. (2009). An item response theory/confirmatory factor analysis of the Autobiographical Memory Test. Memory, 17, 609-623. doi: $10.1080 / 09658210902939348$

Griffith, J. W., Sumner, J. A., Raes, F., Barnhofer, T., Debeer, E., \& Hermans, D. (2012). Current psychometric and methodological issues in the measurement of overgeneral autobiographical memory. Journal of Behavior Therapy and Experimental Psychiatry, 43, S21-S31. doi:10.1016/j.jbtep.2011.05.008

Gutenbrunner, C., Salmon, K., \& Jose, P. E. (2017). Do overgeneral autobiographical memories predict increased psychopathological symptoms in community youth? A 3 year longitudinal investigation. Journal of Abnormal Child Psychology. Advance online publication. doi:10.1007/s10802-017-0278-5

Gutenbrunner, C., Salmon, K., \& Jose, P. E. (2017). What predicts overgeneral memory in youth? Testing the CaR-FA-X model longitudinally in community adolescents. Manuscript under review.

Gutenbrunner, C., Salmon, K., \& Jose, P. E. (2017). Episodic specificity and detail: Investigating longitudinal associations with psychopathology in youth. Manuscript submitted for publication.

Habermas, T., \& Diel, V. (2013). The episodicity of verbal reports of personally significant autobiographical memories: Vividness correlates with narrative text quality more than with detailedness or memory specificity. Frontiers in Behavioral Neuroscience, 7 , 110. doi:10.3389/fnbeh.2013.00110

Hallford, D., \& Mellor, D. (2017). Autobiographical memory specificity and general symptoms of anxiety: Indirect associations through rumination. International Journal of Mental Health, 46, 74-88. doi:10.1080/00207411.2017.1294968 
Hamlat, E. J., Connolly, S. L., Hamilton, J. L., Stange, J. P., Abramson, L. Y., \& Alloy, L. B. (2015). Rumination and overgeneral autobiographical memory in adolescents: An integration of cognitive vulnerabilities to depression. Journal of Youth and Adolescence, 44, 806-818. doi: 10.1007/s10964-014-0090-2

Hankin, B. L., Abramson, L. Y., Moffitt, T. E., Silva, P. A., McGee, R., \& Angell, K. E. (1998). Development of depression from preadolescence to young adulthood: Emerging gender differences in a 10-year longitudinal study. Journal of Abnormal Psychology, 107, 128-140. doi:0.1037/0021-843X.107.1.128

Hermans, D., Vandromme, H., Debeer, E., Raes, F., Demyttenaere, K., Brunfaut, E., \& Williams, J. M. G. (2008a). Overgeneral autobiographical memory predicts diagnostic status in depression. Behaviour Research and Therapy, 46, 668-677. doi:10.1016/j.brat.2008.01.018

Hermans, D., de Decker, A., De Peuter, S., Raes, F., Eelen, P., \& Williams, J. M. G. (2008b). Autobiographical memory specificity and affect regulation: Coping with a negative life event. Depression and Anxiety, 25, 787-792. doi:10.1002/da.20326

Hermans, D., Defranc, A., Raes, F., Williams, J. M. G., \& Eelen, P. (2005). Reduced autobiographical memory specificity as an avoidant coping style. British Journal of Clinical Psychology, 44, 583-589. doi:10.1348/014466505X53461

Heron, J., Crane, C., Gunnell, D., Lewis, G., Evans, J., \& Williams, J. M. G. (2012). 40,000 memories in young teenagers: Psychometric properties of the autobiographical memory est in a UK cohort study. Memory, 20, 300-320. doi:10.1080/09658211.2012.656846

Hipwell, A. E., Sapotichne, B., Klostermann, S., Battista, D., \& Keenan, K. (2011). Autobiographical memory as a predictor of depression vulnerability in girls. Journal of Clinical Child and Adolescent Psychology, 40, 254-265. doi:10.1080/15374416.2011.546037

Hitchcock, C. (2014). Overgeneral memory, trauma, and psychopathology in children (Doctoral thesis). Flinders University, Adelaide, Australia.

Hitchcock, C., Hammond, E., Rees, C., Panesar, I., Watson, P., Werner-Seidler, A., \& Dalgleish, T. (2015). Memory Flexibility training (MemFlex) to reduce depressive symptomatology in individuals with major depressive disorder: Study protocol for a randomised controlled trial. Trials, 16. doi:10.1186/s13063-015-1029-y 
Hitchcock, C., Nixon, R. D. V, \& Weber, N. (2014a). A review of overgeneral memory in child psychopathology. British Journal of Clinical Psychology, 53, 170-193. doi:10.1111/bjc.12034

Hitchcock, C., Nixon, R. D. V., \& Weber, N. (2014b). A longitudinal examination of overgeneral memory and psychopathology in children following recent trauma exposure. Applied Cognitive Psychology, 28, 531-538. doi:10.1002/acp.3027

Hitchcock, C., Werner-Seidler, A., Blackwell, S. E., \& Dalgleish, T. (2017). Autobiographical episodic memory-based training for the treatment of mood, anxiety and stress-related disorders: A systematic review and meta-analysis. Clinical Psychology Review, 52, 92-107. doi:10.1016/j.cpr.2016.12.003

Jing, H. G., Madore, K. P., \& Schacter, D. L. (2016). Worrying about the future: An episodic specificity induction impacts problem solving, reappraisal, and well-being. Journal of Experimental Psychology: General, 145, 402-418. doi: 10.1037/xge0000142

Joormann, J., \& Gotlib, I. H. (2010). Emotion regulation in depression: relation to cognitive inhibition. Cognition \& Emotion, 24, 281-98. doi:10.1080/02699930903407948

Jose, P. E., \& Brown, I. (2008). When does the gender difference in rumination begin? Gender and age differences in the use of rumination by adolescents. Journal of Youth and Adolescence, 37, 180-192. doi:10.1007/s10964-006-9166-y

Jose, P. E., \& Huntsinger, C. S. (2005). Moderation and mediation effects of coping by Chinese American and European American adolescents. The Journal of Genetic Psychology, 166, 16-43. doi:10.3200/GNTP.166.1.16-44

Jung, T., \& Wickrama, K. A. (2008). An introduction to latent class growth analysis and growth mixture modelling. Social and Personality Psychology Compass, 2, 302-317. doi:10.1111/j.1751-9004.2007.00054.x

Kessler, R. C., Amminger, G. P., Aguilar-Gaxiola, S., Alonso, J., Lee, S., \& Ustun, T. B. (2007). Age of onset of mental disorders: A review of recent literature. Current Opinion in Psychiatry, 20, 359-364. doi:10.1097/YCO.0b013e32816ebc8c

Kovacs, M. (1985). The children's depression inventory. Psychopharmacology Bulletin, 21, 995-998.

Kovacs, M. (1992). The Children's Depression Inventory (CDI) Manual. New York, NY: Multi-Health Systems.

Kuyken, W., \& Dalgleish, T. (2011). Overgeneral autobiographical memory in adolescents at risk for depression. Memory, 19, 241-250. doi:10.1080/09658211.2011.554421 
Kuyken, W., Howell, R., \& Dalgleish, T. (2006). Overgeneral autobiographical memory in depressed adolescents with, versus without, a reported history of trauma. Journal of Abnormal Psychology, 115, 387-396. doi:10.1037/0021-843X.115.3.387

Kuyken, W., Watkins, E., Holden, E., \& Cook, W. (2006). Rumination in adolescents at risk for depression. Journal of Affective Disorders, 96, 39-47.

doi:10.1016/j.jad.2006.05.017

Kyung, Y., Yanes-Lukin, P., \& Roberts, J. E. (2016). Specificity and detail in autobiographical memory: Same or different constructs? Memory, 24, 272-284. doi: $10.1080 / 09658211.2014 .1002411$

Lau, J., \& Waters, A. (2017). Annual Research Review: An expanded account of information- processing mechanisms in risk for child and adolescent anxiety and depression. Journal of Child Psychology and Psychiatry, 58, 387-407. doi:10.1111/jcpp.12653

Lemogne, C., Piolino, P., Friszer, S., Claret, A., Girault, N., Jouvent, R., ... \& Fossati, P. (2006). Episodic autobiographical memory in depression: Specificity, autonoetic consciousness, and self-perspective. Consciousness and Cognition, 15, 258-268. doi:10.1016/j.concog.2005.07.005

Levine, B., Svoboda, E., Hay, J. F., Winocur, G., \& Moscovitch, M. (2002). Aging and autobiographical memory: Dissociating episodic from semantic retrieval. Psychology and Aging, 17, 677-689. doi: 10.1037//0882-7974.17.4.677

Little, J. A., \& Rubin, D. B. (2002). Statistical analysis with missing data ( $2^{\text {nd }}$ ed.). Hoboken, NJ: John Wiley and Sons.

Little, P. T. D. (2013). Longitudinal structural equation modelling. New York, NY: Guilford Press.

Lo, Y., Mendell, N. R., \& Rubin, D. B. (2001). Testing the number of components in a normal mixture. Biometrika, 88, 767-778. doi:10.1093/biomet/90.4.991

Lowe, P. A. (2015). The Revised Children's Manifest Anxiety Scale-Second Edition Short Form: Examination of the psychometric properties of a brief measure of general anxiety in a sample of children and adolescents. Journal of Psychoeducational Assessment, 33, 719-730. doi:10.1177/0734282915580763

Lyubomirsky, S., Layous, K., Chancellor, J., \& Nelson, S. K. (2015). Thinking about rumination: The scholarly contributions and intellectual legacy of Susan NolenHoeksema. Annual Review of Clinical Psychology, 11, 1-22. doi:10.1146/annurevclinpsy-032814-112733 
McEvoy, P. M., Watson, H., Watkins, E. R., \& Nathan, P. (2013). The relationship between worry, rumination, and comorbidity: Evidence for repetitive negative thinking as a transdiagnostic construct. Journal of Affective Disorders, 151, 313-320. doi: 10.1016/j.jad.2013.06.014

Moffitt, T. E., Harrington, H., Caspi, A., Kim-Cohen, J., Goldberg, D., Gregory, A. M., \& Poulton, R. (2007). Depression and generalized anxiety disorder: Cumulative and sequential comorbidity in a birth cohort followed prospectively to age 32 years. Archives of General Psychiatry, 64, 651-660. doi:10.1001/archpsyc.64.6.651

Moore, S. A., \& Zoellner, L. A. (2007). Overgeneral autobiographical memory and traumatic events: An evaluative review. Psychological Bulletin, 133, 419-437. doi:10.1037/0033-2909.133.3.419

Morgan, J. (2010). Autobiographical memory biases in social anxiety. Clinical Psychology Review, 30, 288-297. doi:0.1016/j.cpr.2009.12.003

Moulds, M. L., Kandris, E., Starr, S., \& Wong, A. C. (2007). The relationship between rumination, avoidance and depression in a non-clinical sample. Behaviour Research and Therapy, 45, 251-261. doi:10.1016/j.brat.2006.03.003

Muthén, B., \& Muthén, L. K. (2000). Integrating person-centered and variable-centered analyses: growth mixture modelling with latent trajectory classes. Alcoholism: Clinical and Experimental Research, 24, 882-891. doi:10.1111/j.15300277.2000.tb02070.x

Muthén, L. K., \& Muthén, B. O. (1998-2015). Mplus User's Guide. Seventh Edition. Los Angeles, CA: Muthén \& Muthén.

Nelson, K., \& Fivush, R. (2004). The emergence of autobiographical memory: A social cultural developmental theory. Psychological Review, 111, 486-511. doi:10.1037/0033-295X.111.2.486

Nolen-Hoeksema, S. (1991). Responses to depression and their effects on the duration of depressive episodes. Journal of Abnormal Psychology, 100, 569-582. doi:10.1037/0021-843X.100.4.569

Nolen-Hoeksema, S., \& Girgus, J. S. (1994). The emergence of gender differences in depression during adolescence. Psychological Bulletin, 115, 424-443. doi:10.1037/0033-2909.115.3.424

Nolen-Hoeksema, S., \& Watkins, E. R. (2011). A heuristic for developing transdiagnostic models of psychopathology explaining multifinality and divergent 
trajectories. Perspectives on Psychological Science, 6, 589-609. doi: $10.1177 / 1745691611419672$

Nuttall, A. K., Valentino, K., Comas, M., McNeill, A. T., \& Stey, P. C. (2014). Autobiographical memory specificity among preschool-aged children. Developmental Psychology, 50, 1963-1972. doi:10.1037/a0036988

Ono, M., Devilly, G. J., \& Shum, D. H. K. (2016). A meta-analytic review of overgeneral memory: The role of trauma history, mood, and the presence of posttraumatic stress disorder. Psychological Trauma: Theory, Research, Practice, and Policy, 8, 157-164. doi: $10.1037 /$ tra0000027

Orbach, Y., Lamb, M. E., Sternberg, K. J., Williams, J. M. G., \& Dawud-Noursi, S. (2001). The effect of being a victim or witness of family violence on the retrieval of autobiographical memories. Child Abuse and Neglect, 25, 1427-1437. doi:10.1016/S0145-2134(01)00283-6

Ottenbreit, N. D., \& Dobson, K. S. (2004). Avoidance and depression: the construction of the Cognitive-Behavioral Avoidance Scale. Behaviour Research and Therapy, 42, 293313. doi:10.1016/S0005-7967(03)00140-2

Park, R. J., Goodyer, I. M., \& Teasdale, J. D. (2002). Categoric overgeneral autobiographical memory in adolescents with major depressive disorder. Psychological Medicine, 32 , 267-276. doi:10.1017/S0033291701005189

Park, R. J., Goodyer, I. M., \& Teasdale, J. D. (2004). Effects of induced rumination and distraction on mood and overgeneral autobiographical memory in adolescent major depressive disorder and controls. Journal of Child Psychology and Psychiatry and Allied Disciplines, 45, 996-1006. doi:10.1111/j.1469-7610.2004.t01-1-00291.x

Peterson, C., Baker-Ward, L., \& Grovenstein, T. N. (2016). Childhood remembered: Reports of both unique and repeated events. Memory, 24, 240-256. doi:10.1080/09658211.2014.1001991

Pillemer, D. B. (2003). Directive functions of autobiographical memory: The guiding power of the specific episode. Memory, 11, 193-202. doi:10.1080/741938208

Pine, D. S., Cohen, P., Gurley, D., Brook, J., \& Ma, Y. (1998). The risk for early-adulthood anxiety and depressive disorders in adolescents with anxiety and depressive disorders. Archives of General Psychiatry, 55, 56-64. doi:10.1001/archpsyc.55.1.56

Piolino, P., Desgranges, B., \& Eustache, F. (2009). Episodic autobiographical memories over the course of time: Cognitive, neuropsychological and neuroimaging findings. Neuropsychologia, 47, 2314-2329. doi:10.1016/j.neuropsychologia.2009.01.020 
Raes, F., Hermans, D., De Decker, A., Eelen, P., \& Williams, J. M. G. (2003).

Autobiographical memory specificity and affect regulation: An experimental approach. Emotion, 3, 201-206. doi:10.1037/1528-3542.3.2.201

Raes, F., Hermans, D., Williams, J. M. G., Beyers, W., Brunfaut, E., Eelen, P., \& Watson, D. (2006b). Reduced autobiographical memory specificity and rumination in predicting the course of depression. Journal of Abnormal Psychology, 115, 699-704. doi:0.1037/0021-843X.115.4.699

Raes, F., Hermans, D., Williams, J. M. G., Demyttenaere, K., Sabbe, B., Pieters, G., \& Eelen, P. (2005). Reduced specificity of autobiographical memory: A mediator between rumination and ineffective social problem-solving in major depression? Journal of Affective Disorders, 87, 331-335. doi:10.1016/j.jad.2005.05.004

Raes, F., Hermans, D., Williams, J. M. G., \& Eelen, P. (2007). A sentence completion procedure as an alternative to the Autobiographical Memory Test for assessing overgeneral memory in non-clinical populations. Memory, 15, 495-507. doi:10.1080/09658210701390982

Raes, F., Hermans, D., Williams, J. M. G., Geypen, L., \& Eelen, P. (2006a). The effect of overgeneral autobiographical memory retrieval on rumination. Psychologica Belgica, 46, 131-141. doi:10.5334/pb-46-1-2-131

Raes, F., Schoofs, H., Griffith, J. W., \& Hermans, D. (2012). Rumination relates to reduced autobiographical memory specificity in formerly depressed patients following a selfdiscrepancy challenge: The case of autobiographical memory specificity reactivity. Journal of Behavior Therapy and Experimental Psychiatry, 43, 1002-1007. doi:10.1016/j.jbtep.2012.03.003

Raes, F., Verstraeten, K., Bijttebier, P., Vasey, M. W., \& Dalgleish, T. (2010). Inhibitory control mediates the relationship between depressed mood and overgeneral memory recall in children. Journal of Clinical Child \& Adolescent Psychology, 39, 276-281. doi:10.1080/15374410903532684

Raes, F., Watkins, E. R., Williams, J. M. G., \& Hermans, D. (2008). Non-ruminative processing reduces overgeneral autobiographical memory retrieval in students. Behaviour Research and Therapy, 46, 748-756. doi: 10.1016/j.brat.2008.03.003

Rawal, A., \& Rice, F. (2012a). A longitudinal study of processes predicting the specificity of autobiographical memory in the adolescent offspring of depressed parents. Memory, 20, 518-526. doi:10.1080/09658211.2012.683011 
Rawal, A., \& Rice, F. (2012b). Examining overgeneral autobiographical memory as a risk factor for adolescent depression. Journal of the American Academy of Child \& Adolescent Psychiatry, 51, 518-527. doi:10.1016/j.jaac.2012.02.025

Reid, S., Salmon, K., \& Lovibond, P. (2006). Cognitive biases in childhood anxiety, depression, and aggression: Are they pervasive or specific? Cognitive Therapy and Research, 30, 531-549. doi:10.1007/s10608-006-9077-y

Reynolds, C. R., \& Richmond, B. O. (2008). Revised Children's Manifest Anxiety ScaleSecond Edition (RCMAS-2). Los Angeles, CA: Western Psychological Services.

Rothbart, M. K., \& Bates, J. E. (1998). Temperament. In W. Damon, N. Eisenberg (Eds.), Handbook of child psychology: Social, emotional, and personality development (pp. 105-176). New York: Wiley.

Salmon, K., Isler, L., Glynn, R., Mitchell, C., Dewhirst, M., Buxton, B., . . . Jose, P. E. (2017). Caught in the detail: Greater episodic detail in narratives of critical life events predicts an increase in adolescent depression over time. Manuscript under review.

Salmon, K., \& O'Kearney, R. (2014). Emotional memory, psychopathology, and well-being. In P. J. Bauer and R. Fivush (Eds.), The Wiley handbook on the development of children's memory (pp. 743-773). Chichester: John Wiley \& Sons.

Šidák, Z. (1967). Rectangular confidence regions for the means of multivariate normal distributions. Journal of the American Statistical Association, 62, 626-633. doi:10.1080/01621459.1967.10482935

Smets, J., Griffith, J. W., Wessel, I., Walschaerts, D., \& Raes, F. (2013). Depressive symptoms moderate the effects of a self-discrepancy induction on overgeneral autobiographical memory. Memory, 21, 751-761. doi:10.1080/09658211.2012.756039

Söderlund, H., Moscovitch, M., Kumar, N., Daskalakis, Z. J., Flint, A., Herrmann, N., \& Levine, B. (2014). Autobiographical episodic memory in major depressive disorder. Journal of Abnormal Psychology, 123, 51-60. doi: 10.1037/a0035610

Stange, J. P., Hamlat, E. J., Hamilton, J. L., Abramson, L. Y., \& Alloy, L. B. (2013). Overgeneral autobiographical memory, emotional maltreatment, and depressive symptoms in adolescence: Evidence of a cognitive vulnerability-stress interaction. Journal of Adolescence, 36, 20-208. doi: 10.1016/j.adolescence.2012.11.001

Stewart, T., Hunter, S., \& Rhodes, S. (2017). A narrative synthesis of the applicability of the CaR-FA-X model in child and adolescent populations: A systematic review. Memory, 25, 1161-1190. doi:10.1080/09658211.2016.1275699 
Stokes, D. J., Dritschel, B. H., \& Bekerian, D. A. (2004). The effect of burn injury on adolescents autobiographical memory. Behaviour Research and Therapy, 42, 13571365. doi:10.1016/j.brat.2003.10.003

Sumner, J. A. (2012). The mechanisms underlying overgeneral autobiographical memory: An evaluative review of evidence for the CaR-FA-X model. Clinical Psychology Review, 32, 34-48. doi:10.1016/j.cpr.2011.10.003

Sumner, J. A., Griffith, J. W., \& Mineka, S. (2010). Overgeneral autobiographical memory as a predictor of the course of depression: A meta-analysis. Behaviour Research and Therapy, 48, 614-625. doi:10.1016/j.brat.2010.03.013

Sumner, J. A., Griffith, J. W., Mineka, S., Rekart, K. N., Zinbarg, R. E., \& Craske, M. G. (2011). Overgeneral autobiographical memory and chronic interpersonal stress as predictors of the course of depression in adolescents. Cognition and Emotion, 25, 183-192. doi:10.1080/02699931003741566

Sumner, J. A., Mineka, S., Adam, E. K., Craske, M. G., Vrshek-Schallhorn, S., WolitzkyTaylor, K., \& Zinbarg, R. E. (2014). Testing the CaR-FA-X model: Investigating the mechanisms underlying reduced autobiographical memory specificity in individuals with and without a history of depression. Journal of Abnormal Psychology, 123, 471486. doi:10.1037/a0037271

Swales, M., Williams, J. M. G., \& Wood, P. (2001). Specificity of autobiographical memory and mood disturbance in adolescents, Cognition and Emotion, 15, 321-331. doi:10.1080/0269993004200132

Thapar, A., Collishaw, S., Pine, D. S., \& Thapar, A. K. (2012). Depression in adolescence. The Lancet, 379, 1056-1067. doi:10.1016/S0140-6736(11)60871-4

Treynor, W., Gonzalez, R., \& Nolen-Hoeksema, S. (2003). Rumination reconsidered: A psychometric analysis. Cognitive Therapy and Research, 27, 247-259. doi:10.1023/A:1023910315561

Tulving, E. (2001). Episodic memory and common sense: How far apart? Philosophical Transactions of the Royal Society of London. Series B, 356(1413), 1505-1515. doi:10.1098/rstb.2001.0937

Tulving, E. (2002). Episodic memory: From mind to brain. Annual Review of Psychology, 53, 1-25. doi:10.1146/annurev.psych.53.100901.135114

Van Vreeswijk, M. F., \& De Wilde, E. J. (2004). Autobiographical memory specificity, psychopathology, depressed mood and the use of the Autobiographical Memory Test: 
A meta-analysis. Behaviour Research and Therapy, 42, 731-743. doi:10.1016/S00057967(03)00194-3

Vanderveren, E., Bijttebier, P., \& Hermans, D. (2017). The importance of memory specificity and memory coherence for the self: Linking two characteristics of autobiographical memory. Frontiers in Psychology, 8. doi:10.3389/fpsyg.2017.02250

Valentino, K. (2011). A developmental psychopathology model of overgeneral autobiographical memory. Developmental Review, 31, 32-54. doi:10.1016/j.dr.2011.05.001

Valentino, K., Bridgett, D. J., Hayden, L. C., \& Nuttall, A. K. (2012). Abuse, depressive symptoms, executive functioning, and overgeneral memory among a psychiatric sample of children and adolescents. Journal of Clinical Child and Adolescent Psychology, 41, 491-498. doi:10.1080/15374416.2012.660689

Valentino, K., Nuttall, A. K., Comas, M., McDonnell, C. G., Piper, B., Thomas, T. E., \& Fanuele, S. (2014). Mother-child reminiscing and autobiographical memory specificity among preschool-age children. Developmental Psychology, 50, 1197-1207. doi: $10.1037 / \mathrm{a} 0034912$

Valentino, K., Toth, S. L. \& Cicchetti, D. (2009). Autobiographical memory functioning among abused, neglected, and nonmaltreated children: the overgeneral memory effect. Journal of Child Psychology and Psychiatry, 50, 1029-1038. doi:10.1111/j.14697610.2009.02072.x

Vrielynck, N., Deplus, S., \& Philippot, P. (2007). Overgeneral autobiographical memory and depressive disorder in children. Journal of Clinical Child and Adolescent Psychology. 36, 95-105. doi:10.1080/15374410709336572

Wang, Q. (2004). The emergence of cultural self-constructs: autobiographical memory and self-description in European American and Chinese children. Developmental Psychology, 40, 3-15. doi:10.1037/0012-1649.40.1.3

Wang, Q., \& Conway, M. A. (2004). The stories we keep: Autobiographical memory in American and Chinese middle-aged adults. Journal of Personality, 72, 911-938. doi:10.1111/j.0022-3506.2004.00285.x

Waters, T. (2014). Relations between the functions of autobiographical memory and psychological wellbeing. Memory, 22, 265-275. doi:10.1080/09658211.2013.778293

Waters, T., Bauer, P., \& Fivush, R. (2014). Autobiographical memory functions served by multiple event types. Applied Cognitive Psychology, 28, 185-195. doi:10.1002/acp.2976 
Watkins, E. R. (2015). Psychological treatment of depressive rumination. Current Opinion in Psychology, 4, 32-36. doi:10.1016/j.copsyc.2015.01.020

Watkins, E. R., Baeyens, C. B., \& Read, R. (2009). Concreteness training reduces dysphoria: Proof-of-principle for repeated cognitive bias modification in depression. Journal of Abnormal Psychology, 118, 55-64. doi:10.1037/a0013642

Watkins, E. R., \& Moulds, M. (2005). Distinct modes of ruminative self-focus: Impact of abstract versus concrete rumination on problem solving in depression. Emotion, 5, 319-328. doi:10.1037/1528-3542.5.3.319

Watkins, E. R., Teasdale, J., \& Baker, T. B. (2001). Rumination and overgeneral memory in depression: Effects of self-focus and analytic thinking. Journal of Abnormal Psychology, 110, 353-357. doi:10.1037/0021-843X.110.2.333

Wechsler, D. (2004). Wechsler Intelligence Scale for Children - Fourth UK edition. London: Harcourt Assessment.

Wessel, I., Meeren, M., Peeters, F., Arntz, A., \& Merckelbach, H. (2001). Correlates of autobiographical memory specificity: The role of depression, anxiety and childhood trauma. Behaviour Research and Therapy, 39, 409-421. doi:10.1016/S00057967(00)00011-5

Williams, J. M. G. (1996). Depression and the specificity of autobiographical memory. In D. C. Rubin (Ed.), Remembering our past: Studies in autobiographical memory (pp. 244267). Cambridge, UK: Cambridge University Press.

Williams, J. M. G., Barnhofer, T., Crane, C., Herman, D., Raes, F., Watkins, E., \& Dalgleish, T. (2007). Autobiographical memory specificity and emotional disorder. Psychological Bulletin, 133, 122-148. doi:10.1037/0033-2909.133.1.122

Williams, J. M. G., \& Broadbent, K. (1986). Autobiographical memory in suicide attempters. Journal of Abnormal Psychology, 95, 144-149. doi:10.1037/0021-843X.95.2.144

Zlomuzica, A., Dere, D., Machulska, A., Adolph, D., Dere, E., \& Margraf, J. (2014). Episodic memories in anxiety disorders: clinical implications. Frontiers in Behavioral Neuroscience, 8. doi:10.3389/fnbeh.2014.00131 


\section{Appendix}

Appendix A: Autobiographical Memory Test (AMT) booklet with sample memory recording page

\section{MEMORY BOOKLET}

Hello. We come from Victoria University of Wellington and we are very interested in learning how people of your age think about themselves and their lives. We want to find this out so that we can understand better how to help kids feel good about themselves and their world as they get older. We are going to ask you a whole lot of questions today, and we hope that you will find these interesting as you fill them out.

Remember - nothing you say goes to anyone else (other than the researchers) unless we are very worried about your safety. Also, once we have your forms, with your names, we take your name off and use a number only.

Thank you very much for helping us.

We are interested in your memory for events that have happened in your life. For each of the following words we would like you to think of an event that happened to you which the word reminds you of. The event could have happened recently (e.g., yesterday, last week...) or a long time ago. It might be an important event, or a trivial event.

The memory you write down should be for a real event. So if we said "good" - it would not be OK to say "I always enjoy a good party" because this does not mention a specific event. But it would be OK to say "I had a good time at Jane's party" because that is a real event.

\section{Please don't use the same event more than once.}

After I read each word, you will have about a minute to think about, and write down your memory. We'll tell you when to start and when it's time to stop. You can use as many or as few lines as you want. You don't have to fill in all the lines. Don't turn the page until we tell you to.

Sample memory record page (identical for all ten cue words; separate A5 size page for each cue)

Happy 
Appendix B: Questionnaire booklet

Date

Code Number

QUESTION BOOKLET 
Kids sometimes have different feelings and ideas. This form lists the feelings and ideas in groups of 3 sentences. From each group of three sentences, pick one sentence that describes you best for the past two weeks. After you pick a sentence from the first group, go on to the next group.

There is no right or wrong answer. Just pick the sentence that best describes the way you have been recently. Put a mark like this X next to your answer. Put the mark on the line next to the sentence you pick. Choose only ONE in each group of 3 sentences.

\begin{tabular}{|c|c|}
\hline $\begin{array}{l}\text { Item } 1 . \\
\quad \text { I am sad once in a while } \\
\text { I am sad many times } \\
\text { I I am sad all the time }\end{array}$ & 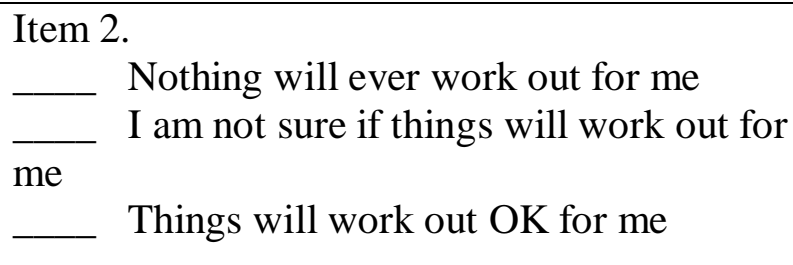 \\
\hline $\begin{array}{l}\text { Item } 3 . \\
\text { I do most things OK } \\
\text { I do many things wrong } \\
\text { I do everything wrong }\end{array}$ & $\begin{array}{l}\text { Item } 4 . \\
\text { I have fun in many things } \\
\text { I have fun in some things } \\
\text { Nothing is fun at all }\end{array}$ \\
\hline $\begin{array}{l}\text { Item } 5 . \\
-\quad \text { I am important to my family } \\
\frac{\text { I amily }}{\text { famot sure if I am important to my }} \\
\quad \text { My family is better off without me }\end{array}$ & $\begin{array}{l}\text { Item } 6 \\
\quad \text { I hate myself } \\
\text { I do not like myself } \\
\text { I like myself }\end{array}$ \\
\hline $\begin{array}{ll}\text { Item } 7 & \\
& \text { I feel grumpy all the time } \\
& \text { I feel grumpy many times } \\
& \text { I am almost never grumpy }\end{array}$ & $\begin{array}{l}\text { Item } 8 \\
- \text { I cannot make up my mind about things } \\
\text { things } \text { It is hard to make up my mind about } \\
\text { I make up my mind about things easily }\end{array}$ \\
\hline 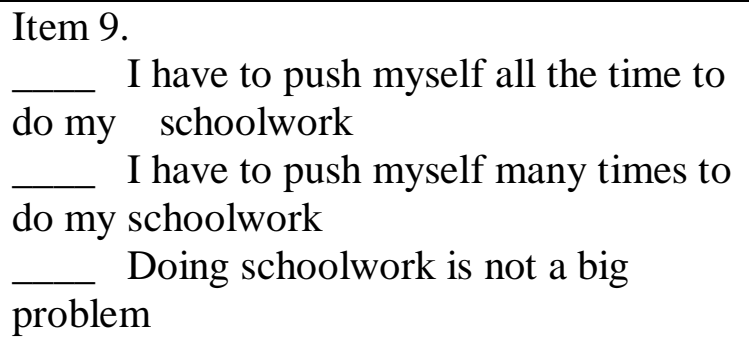 & $\begin{array}{l}\text { Item } 10 . \\
\\
\\
\\
\text { I I am tired once in a while } \\
\text { I am tired all the time }\end{array}$ \\
\hline $\begin{array}{l}\text { Item } 11 . \\
\square \quad \text { Most days I do not feel like eating } \\
\text { Many days I do not feel like eating } \\
\text { I eat pretty well }\end{array}$ & $\begin{array}{l}\text { Item } 12 . \\
\text { I do not feel alone } \\
\text { I feel alone many times } \\
\text { I feel alone all the time }\end{array}$ \\
\hline
\end{tabular}


We are interested in what people think about themselves. Circle one answer for each sentence.

1. When something good happens, I feel thankful. YES NO

2. When I am happy, I show it on my face. YES NO

3. Often I feel sick in my stomach. YES NO

4. I am nervous. $\quad$ YES NO

5. I often worry about something bad happening to me. YES NO

6. I fear other kids will laugh at me in class. YES NO

7. I have too many headaches. YES NO

8. I think I am happier than most of my friends. YES NO

9. I worry that others do not like me. YES NO

10. I wake up scared sometimes. $\quad$ YES NO

11. I get nervous around people. YES NO

12. I feel someone will tell me I do things the wrong way. YES NO

13. I fear other people will laugh at me. $\quad$ YES NO

14. When I do something hard, I feel proud of myself. $\quad$ YES NO

15. I am a happy person. $\quad$ YES NO 
We are interested in how you feel. This is not a test: there are no right or wrong answers. When people feel happy or sad, they do and think different things. What about you? What do you do and think when you feel happy or sad? For each question, please mark what you usually do, not what you think you should do.

\begin{tabular}{|c|c|c|c|c|}
\hline & $\begin{array}{c}\text { Almost } \\
\text { never }\end{array}$ & $\begin{array}{l}\text { Some } \\
\text {-times }\end{array}$ & Often & $\begin{array}{l}\text { Almost } \\
\text { always }\end{array}$ \\
\hline $\begin{array}{l}\text { 1. When I am happy, I like to go to the movies } \\
\text { with my friends. }\end{array}$ & 1 & 2 & 3 & 4 \\
\hline 2. When I am sad, I try to ignore my feelings. & 1 & 2 & 3 & 4 \\
\hline $\begin{array}{l}\text { 3. When I am sad, I try to ignore or get away from } \\
\text { my problems. }\end{array}$ & 1 & 2 & 3 & 4 \\
\hline $\begin{array}{l}\text { 4. When I am sad, I go away by myself and think } \\
\text { about why I feel this way. }\end{array}$ & 1 & 2 & 3 & 4 \\
\hline $\begin{array}{l}\text { 5. When I am sad, I talk about it with someone who } \\
\text { can help me feel better. }\end{array}$ & 1 & 2 & 3 & 4 \\
\hline $\begin{array}{l}\text { 6. When I am sad, I watch TV or play video games } \\
\text { so I don't think about how sad I am. }\end{array}$ & 1 & 2 & 3 & 4 \\
\hline 7. When I am sad, I think I'm ruining everything. & 1 & 2 & 3 & 4 \\
\hline $\begin{array}{l}\text { 8. When I am sad, I remind myself that this feeling } \\
\text { will go away. }\end{array}$ & 1 & 2 & 3 & 4 \\
\hline 9. When I am happy, I read a book or a magazine. & 1 & 2 & 3 & 4 \\
\hline $\begin{array}{l}\text { 10. When I am sad, I try to pretend there isn't a } \\
\text { problem. }\end{array}$ & 1 & 2 & 3 & 4 \\
\hline $\begin{array}{l}\text { 11. When I am sad, I stay away from the person or } \\
\text { situation that is causing the problem. }\end{array}$ & 1 & 2 & 3 & 4 \\
\hline 12. When I am sad, I do something I enjoy. & 1 & 2 & 3 & 4 \\
\hline $\begin{array}{l}\text { 13. When I am sad, I think about how angry I am } \\
\text { with myself. }\end{array}$ & 1 & 2 & 3 & 4 \\
\hline $\begin{array}{l}\text { 14. When I am sad, I make up my mind that things } \\
\text { are OK even if they're not. }\end{array}$ & 1 & 2 & 3 & 4 \\
\hline $\begin{array}{l}\text { 15. When I am sad, I avoid thinking about how I } \\
\text { feel. }\end{array}$ & 1 & 2 & 3 & 4 \\
\hline 16. When I am sad, I am afraid to think about my & 1 & 2 & 3 & 4 \\
\hline
\end{tabular}




\begin{tabular}{|c|c|c|c|c|}
\hline feelings. & & & & \\
\hline $\begin{array}{l}\text { 17. When I am sad, I ask a friend, parent or teacher } \\
\text { to help me solve my problem. }\end{array}$ & 1 & 2 & 3 & 4 \\
\hline $\begin{array}{c}\text { 18. When I am happy, I like to listen to my } \\
\text { favourite music. }\end{array}$ & 1 & 2 & 3 & 4 \\
\hline $\begin{array}{l}\text { 19. When I am sad, I think there must be something } \\
\text { wrong with me or I wouldn't feel this way. }\end{array}$ & 1 & 2 & 3 & 4 \\
\hline $\begin{array}{l}\text { 20. When I am sad, I go to my favourite place to get } \\
\text { my mind off my feelings. }\end{array}$ & 1 & 2 & 3 & 4 \\
\hline $\begin{array}{l}\text { 21. When I am sad, although things are bad, I } \\
\text { choose to believe that things are good. }\end{array}$ & 1 & 2 & 3 & 4 \\
\hline $\begin{array}{l}\text { 22. When I am sad, I avoid something that is } \\
\text { making me upset. }\end{array}$ & 1 & 2 & 3 & 4 \\
\hline $\begin{array}{l}\text { 23. When I am sad, I think about all of my failures, } \\
\text { faults, and mistakes. }\end{array}$ & 1 & 2 & 3 & 4 \\
\hline $\begin{array}{l}\text { 24. When I am sad, I decide that things are fine, } \\
\text { even though I know they're not. }\end{array}$ & 1 & 2 & 3 & 4 \\
\hline $\begin{array}{l}\text { 25. When I am sad, I think of a way to make my } \\
\text { problem better. }\end{array}$ & 1 & 2 & 3 & 4 \\
\hline $\begin{array}{l}\text { 26. When I am sad, I think why can't I handle } \\
\text { things better? }\end{array}$ & 1 & 2 & 3 & 4 \\
\hline $\begin{array}{l}\text { 27. When I am sad, I do something fun with a } \\
\text { friend. }\end{array}$ & 1 & 2 & 3 & 4 \\
\hline $\begin{array}{l}\text { 28. When I am happy, I think about how many } \\
\text { things I like to do. }\end{array}$ & 1 & 2 & 3 & 4 \\
\hline
\end{tabular}




\section{$\underline{\text { Directions }}$}

Below you will find a series of statements that people might use to describe themselves. The statements refer to a wide number of activities and attitudes.

For each statement, please circle the answer that best describes how true each statement is for you. There are no right or wrong answers. People are very different in how they feel about these statements. Please circle the first answer that comes to you.

You will use the following scale to describe how true or false a statement is about you:

Circle number:

1
2
3
4
5

If the statement is:

Almost always untrue of you

Usually untrue of you

Sometimes true, sometimes untrue of you

Usually true of you

Almost always true of you

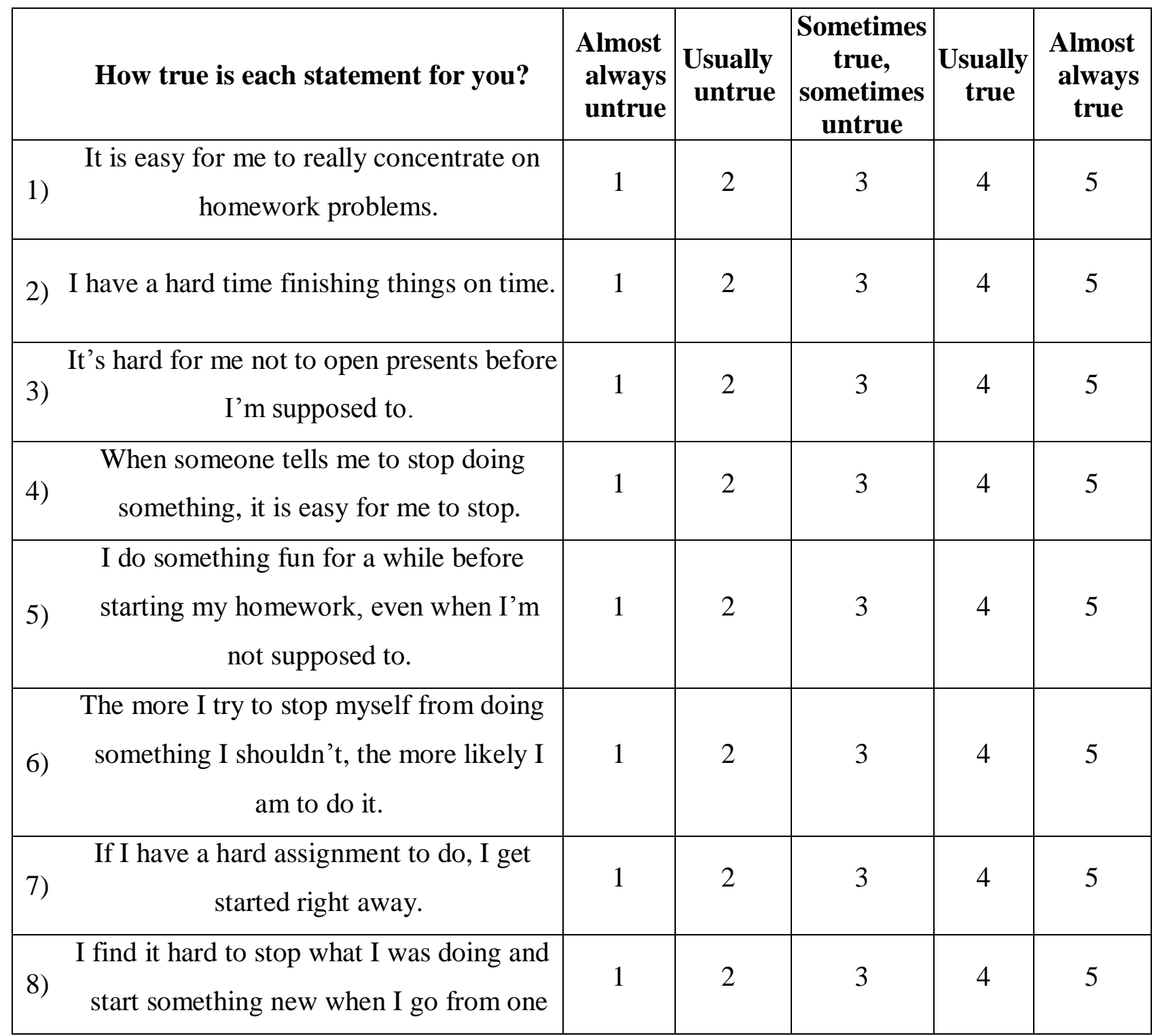




\begin{tabular}{|c|c|c|c|c|c|}
\hline subject to another at school. & & & & & \\
\hline $\begin{array}{l}\text { 9) When trying to study, I have difficulty } \\
\text { tuning out background noise and } \\
\text { concentrating. }\end{array}$ & 1 & 2 & 3 & 4 & 5 \\
\hline 10) I finish my homework before the due date. & 1 & 2 & 3 & 4 & 5 \\
\hline $\begin{array}{l}\text { 11) I am good at keeping track of several } \\
\text { different things that are happening around } \\
\text { me. }\end{array}$ & 1 & 2 & 3 & 4 & 5 \\
\hline 12) It's easy for me to keep a secret. & 1 & 2 & 3 & 4 & 5 \\
\hline $\begin{array}{l}\text { 13) I put off working on projects until right } \\
\text { before they're due. }\end{array}$ & 1 & 2 & 3 & 4 & 5 \\
\hline $\begin{array}{l}\text { 14) I pay close attention when someone tells } \\
\text { me how to do something. }\end{array}$ & 1 & 2 & 3 & 4 & 5 \\
\hline $\begin{array}{l}\text { 15) I tend to get in the middle of one thing, } \\
\text { then go off and do something else. }\end{array}$ & 1 & 2 & 3 & 4 & 5 \\
\hline 16) I can stick with my plans and goals. & 1 & 2 & 3 & 4 & 5 \\
\hline
\end{tabular}


Appendix C: Autobiographical memory coding scheme

\section{Autobiographical Memory Test (AMT) 11-Class Coding Procedure Gutenbrunner, Glynn, Allan, Salmon \& Jose (2014)}

- This coding scheme is an adapted version of scoring procedures used in research by Heron et al. (2012) and sourced through personal communication with Catherine Crane

\section{General Notes:}

Children sometimes respond with mixed tenses. In this case use your judgement on how to code the memory while making as few inferences as possible.

Examples:

I feel happy when I play with my dog yesterday (specific)

I felt excited when I go to my friend's house (categoric)

Specific (SPSS code 1)

An event that occurred on a particular occasion and lasted less than one day. It can often be located in a time or place, but this information does not need to have been provided by participants.

Examples:

On my $13^{\text {th }}$ birthday

During my sleepover last week.

When I came second in a hurdles race

When I was told my Grandad had died

When I found out we were going to Australia

When I was on the plane on the way to Australia

\section{Extended (SPSS code 2)}

An event that lasted longer than 1 day. Note: the memory must be referring to an event. Includes holidays, deaths, parental divorce or separation, child or others moving towns, being bullied, school holidays, school years and other extended periods with time boundaries e.g., "when my cousins were living with us". Trips - use your judgement, is it a place they are likely to travel to and from in less than 1 day?

Examples:

When I was on holiday in Fiji

When I was being bullied

When my parents split up

Categoric (SPSS code 3)

Summaries of a class of events or repeated events. Use your judgement - is it likely that this event would be one that happened repeatedly for a child in this age range?

Examples:

Playing rugby 
When I get home from school

When I get into a fight with my sister

\section{Specific OR Extended (SPSS code 4)}

Memories of a specific event which could have lasted less than 24 hours or could have lasted more than 24 hours and it is not possible to tell from the response. Also include memories that are ambiguous with regards to whether participant referred to specific point in time that may represent the beginning of a subsequently mentioned extended period e.g., "I was sad when Amy left to go to Germany for 6 weeks."

Examples:

When I went camping last weekend

When I went to Palmerston North

When my family were visiting

Specific OR Categoric (SPSS code 5)

Memories of an event that lasted less than 24 hours and may have occurred once or may have occurred more than once and it is not possible to tell from the response

Examples:

Reading my book

Going to the park

In the hot pools at Hamner Springs

\section{Extended AND Categoric (SPSS code 6)}

A response that contains a categoric memory within an extended time period. This extended period may be while overseas, when living in a different city, when in a different school year, during the school holidays

Examples:

On holiday I read a book by the pool

In year 7 when I played soccer

When I lived in Christchurch and I hung out with my friends

\section{Semantic Associate (SPSS code 7)}

A response that is derived from general semantic knowledge rather than a personal memory. Child has not provided a memory of an event. Include responses when something didn't happen e.g., "that I didn't get to say goodbye to my cat" (But does not include "that I didn't get injured when I almost got hit by a car" - this is a specific event). Also includes when participants say they always feel a certain way or feel it every day. May be present tense, but not referring to a specific event that happened to child in the past.

Examples:

I feel lucky to have a family that love me

I always feel lonely

I feel lucky to live in New Zealand

I felt angry that I didn't get to properly say goodbye to my Granddad

My dog 
Future Oriented (SPSS code 8)

Responses that refer to an event that has not yet happened. This event could be specific (i.e. confined to one day) or extended (i.e. will last longer than a day) but it must be an event that the child is going to experience in the future.

Examples:

I'm excited about going to Granddad's on the weekend

I'm scared to do the maths test tomorrow

Incomplete Responses (SPSS code 9)

Partial or incomplete responses that give enough detail to suggest the child had thought of a memory but not enough detail to code. It may seem as though the child ran out of time to complete the response. Note: if the response does not contain any detail about the memory code as Omission (code 10) e.g., "I was happy when"

Examples:

I get angry when my brother

I am proud when I do

I was lonely when I went to

Omissions (SPSS code 10)

Participant does not provide a response or cannot retrieve a memory for the cue. Includes responses that have been started but no memory content has been included e.g., "I feel happy when". Also includes responses that a child has "never" felt that way

Examples:

"I never feel angry"

"I felt happy when"

"N/A"

Repeated Memory (SPSS code 11)

Participant has already provided this response to a different cue 
Hi, I'm , and I'm here with some other people from the university. Your parents have said you can help us out with some research we are doing on how kids remember and think about things. Is that OK? Alright, if you could please follow me, we just have to collect some other students. We'll tell you more about what you'll be doing when we have everyone together as a group.

\section{Event Script}

(once students are seated in test room)

Hi Everyone, my name is , you may have already met (other researcher's names) when we came and collected you. We're from Victoria University, and we're here today because we are interested in finding out how students remember some events in their lives and think about things.

The first thing we're going to ask you to do is read through some info about the study. Your parent has said its fine for you to take part. If you think that's ok then can you sign, too? Remember, everything you write here is private. We have given you two copies so that you can keep this info as well.

[collect information/assent sheets off students as they finish. Make sure that they keep one copy]

OK, thanks everyone for signing that for us. Now we're going to start. There are two parts to our session today. First we'll do the remembering part and after that we'll answer some questions about what we think and feel.

First things first, we're going to give you a booklet. Please don't look through it - all I want you to do is read the first page. Then I want you to turn to the second page and just write down your name, your school, your date of birth and your culture or ethnicity. Remember even though this might feel like a test with us being all serious, it's NOT! There are no right answers or wrong answers; we just want to know your view.

$\mathrm{OK}$, is your name on your booklet now? Great! We're going to start the first part of our session in a moment, but I want you to remember that if there is anything you want to know at any time while you're here, just say! We want you to feel happy and comfortable being here. Also, if you feel uncomfortable at any time during this session and no longer want to stay just let us know and you can go back to class.

Even though this isn't a test, it is important that you listen carefully to what we say and concentrate on your own work. To help everyone else listen and concentrate, it's important that you don't speak to each other and don't discuss your answers.

OK, if you could all turn to the second page I'm going to start reading the instructions for what we are going to do. Are you ready? OK! 
[Read through instructions for the memory task. Stop frequently to make sure students understand the task. Emphasize that they should wait for you to say each word and that they shouldn't read ahead. Tell students that spelling doesn't matter. Remember to provide very clear "go" and "stop" signals].

Great! We've finished the remembering part! What I'd like for you to do now is wait for one of us to come and collect your memory booklet and give you a new booklet. Please don't open the booklet - just leave it on the front page while I tell you about what we're going to do next.

[Researchers should collect memory booklets one at a time, and replace it with a question booklet that you have filled in with the appropriate code number]

In this booklet we're going to answer some questions about what we think and feel. I'm going to read through the instructions and questions with you. Just like before, we'd like everyone to be quiet so that we can focus on answering their own questions. If you get lost or want to know something just ask one of the researchers to help you.

[Read through questions and response options. For questionnaires with scales/many response options read through all the options for the first item (at least) to show children how to respond. Make fun comments about progress. "phew - that one took a bit of thinking." "yes! Only one to go" "this one is fun" "this one asks some hard questions but we'll take it slow." Remind children periodically that this is not a test. No right or wrong answer]

Phew!! We've finished the question part. Thanks so much for all your effort! We're just going to come and collect your booklet off you now.

[collect booklets. If student is NOT in pilot study, give them a copy of the informal debriefing]

Debriefing:

Thanks so much for coming in today! We really hope that you enjoyed yourself. Now I'm going to tell you what happens next. When we finish at [School name] today we take all of these booklets back to the university. The first thing we do it take your name off the booklet so that your answers do not have your name attached to them. Instead, your answers become part of a big group of answers that we use to explore how kids think in general and how their thinking links in with what they remember.

When we finish this study we will send a letter to your school and to you and your parents. This letter will tell you about what we have learned about how kids think and remember things. This letter will not tell your parents or teachers how you did - remember your answers will be combined with other kids answers to create a big group that we will explore.

Do you have any questions about what you did today or what will happen in the future?

Thanks for helping us out! [researcher's name] will take you back to class now. 
- This coding scheme was developed by Kyung et al. (2016), and obtained through personal communications with John Roberts.

\section{Coding Different Properties (Details) of Specific Memory}

Here are the criteria for rating responses that are identified as a specific memory (coded 5) from the previous step. In order to give codings regarding the nature of specific memories, we would be looking at the level of details the response has.

We would only be looking at the level of details within descriptions of a specific event within one day. In order to figure out the level of details of each specific memory, the appropriate, specific part of the answer is to be identified first. Since some responses that are coded as specific memories have some part of the answer that are not descriptions of specific event pertaining to a one-day period, these parts that are irrelevant must be taken out of consideration when rating the level of details of the memory.

Thus, the first step would be to identify the part of response to be coded. Next, the level of details of the appropriate part of the answer can be coded.

\section{(2) Coding level of details}

Once the appropriate part is identified, the level of details in the part can be coded.

In order to code the level of details for each specific response, the number of "units" in each answer part will be counted and summed up. Units can be seen as meaningful measure of how much information or detail the response has. A unit can count information regarding the time when the event happened, place where it happened, who is(are) involved in the event, what has happened, which things are involved, etc. It can also count further information on the manner how the event happened, qualify the things that are involved, count in what is being said, or count in emotional responses during the event.

We can count the total number of units in a response (or response part) by summing up units across 10 categories of time, place, people, objects/events, actions/happenings, manner, qualifiers, quotations, cause/intentions, and emotional responses. For example, a response (part) can have 2 units of time, 1 units of place, and 1 unit of action or happening, summing up to 4 total units.

\section{a. Time}

The following are examples of words that describe time, each considered as 1 unit of time.

When I was in $5^{\text {th }}$ grade, at noon, last summer, on Saturday, when I was living in the U.S.

There can be multiple expressions regarding time in a single answer. Count the number of expressions describing the time when the event happened and add up. 
At $10 o^{\prime}$ 'clock on a Sunday when I was in $6^{\text {th }}$ grade (3 units)

May $5^{\text {th }}$, Saturday (2 units)

Count units as pertaining to the different levels of more than a year/ year/ more than a day/ day of week/ less than a day. Thus, the following example can be counted accordingly as follows:

At 11:20 am/ on Saturday, / June $5^{\text {th }}$, in the summer of/ 1997/ in my childhood (5 units)

-Exceptions. However, note the cases that do not count as units because they are not considered as giving information as to when the event happened. In the following cases, 'one day' and 'one time' does not give any concrete information regarding time, so it is not counted as a unit.

One day I ran into a friend of mine on the subway. $(x)$

There was one time when I had to tell my parents to come to school because of a misunderstanding between the teacher and $I .(x)$

\section{b. Place}

The following are examples of words that describe place.

At home, in school, on the way home, on the street, at a party, at the grocery store, at the conference, in school playground, in the swimming pool, ...

If there are multiple descriptions regarding place, multiple units can be scored.

At my aunt's house near Lake Chungju (2 units)

\section{c. People}

When the response mentions people other than the respondent him/herself, count units accordingly. The following examples are one units each.

Mother, brother, friends, younger sister, oldest brother, cousin, teacher, grandmother, with my boyfriend, someone, the school principal, a stranger

If there are more than one expression as in the following example, more than one unit can be counted. The following example has 2 units regarding people.

with my brother and cousin

Note that words that point to the respondent himself ('I' or 'me') or a nonspecified 'we' do not count as units in this category. (Unless it is specified who is meant by 'we'). I was excited to go on the class field trip. (0 unit for people)

We went on a field trip. (0 unit for people)

My class went on a field trip to Amsa-dong. We were very excited on the way there. (1 unit for people)

\section{d. Objects or events}


Objects or events (usually in the form of noun) that are involved in the description of the event can be counted as in units. The following examples have 1 unit each.

bike, guitar, class, video game, Tetris game, soccer game, blue jeans, midterm exam, math contest, Baduk contest, entrance exam, birthday party, a date, TV program, midterm, midterm exam, art class, ... (1 unit each) In the following example, 2 units can be given.

Candies and chocolates, afterparty of our concert, ... (2 units each)

\section{e. Actions and happenings}

Words or phrases that describe what was done and what happened (usually in the form of verb or verbal phrases) can be counted as units accordingly. The following examples are all 1 unit each.

Talking, eating, fighting, sleeping, buying, going, having a fight, having a meal, going shopping, going skiing, having a conversation, taking an exam, getting good grades, winning a prize, being complimented, falling down, playing soccer, ... (1 unit each)

There can be multiple expressions of action or happenings. The following examples have multiple units regarding actions or happenings.

We went to see the movies, and afterwards went shopping.(2 units for actions)

After taking several classes in a row, I had to go to my part-time job, and then had to do chores when I got back home.(3 units for actions)

In case of a negative form of verb, saying that something did not happen, it will always be counted as 1 unit for the negated part.

I waited for my friend at the bus stop, but she didn't show upfor an hour. (1 unit for the boldfaced part, 4 units total)

-Exceptions. In the following cases, meeting with and going themselves are not counted as actions/happenings, but rather the information is counted as people or place.

I met with a friend and chatted for an hour. (I chatted for an hour with a friend)

We went to the meeting and listened to a guest speaker.(We listened to a guest speaker at the meeting)

\section{f. Manner}

Certain words or phrases can describe the manner which the act or event was done.

My cousin ate up the meal very neatly and my parents complimented on him. (1 unit for manner)

A kid in my class made fun of my height, and I was so angry that I beat him up till his nose bled. (1 unit for manner)

One of my classmates showed my report card to other kids as a prank. (1 unit for manner)

-Exception. Note that expressions that are used without much addition to the meaning, such as 'very' or 'really' are not counted as units. 


\section{g. Qualifiers}

When certain words or phrases describe the property of other unit pieces to give further details or identify further what kind of things they are, count the qualifier units accordingly. Qualifiers can elaborate on time, place, people, objects/events, or actions/happenings. In the following examples, the parts that are in bold print are given 1 unit each for having a qualifying word or phrase. (Although the whole part of example listed below would have more units when counting units for things)

On a breezy Sunday (1 qualifier unit, 2 units total; elaborating on time)

At a famous Italian restaurant (1 qualifier unit, 2 units total; elaborates on place)

The girl I had admired sat next to me on the bus. (1 qualifier unit, 5 units total; elaborates on people)

A friend that I went to same kindergarten with (1 qualifier unit, 2 units total; elaborates on people)

A lengthy discussion (1 qualifier unit, 2 units total; elaborates on object/event)

A beginner-level art class (1 qualifier unit, 2 units total; elaborates on objects/event)

Presidential Cup soccer game (1 qualifier unit, 2 units total; elaborates on objects/events)

The toy that I really wanted to have (1 qualifier unit, 2 units total; elaborates on object/event) In the following examples, multiple qualifier units are added up.

Kids in the neighbourhood admired the pretty new bike my father bought me. (3 qualifier units, 7 units total)

Jina really admired my silver bracelet that I bought at a flea market (2 qualifier unit, 5 units total)

\section{h. Quotations/ thoughts}

When there are quoted phrases or sentences of what is being said or what is being thought during the event, count one unit every time a quote or thought content is included. However, note that, if the quoted part itself includes some specific information about what happened in the one-day period, count according units in addition to the quote being counted.

After having a bad score on the math contest, I was really dejected. My teacher told me then,

"You are a bright student who just takes a little more time to shine." (1 quotation unit)

Father told my brother and I that grandma had passed away.(1 quotations having 2 units in it)

When grandmother passed away, the thought that I couldn't see her any more made me say (1 qualifier unit, 5 units total)

Losing at the final round of Baduk contest, I thought that I will definitely do better next time. (1 unit for thought, 5 units total)

\section{i. Causes/ intentions}


Sometimes the causes of certain part of an event, intentions for actions, thoughts that one has that is involved in the event itself are described. These are also giving detailed information, and can be thought of as having units of detail.

Mother scolded me because of my bad grades. (1 unit for cause, 3 units total)

Mother scolded me because of my bad grades and my attitude.(2 units for cause, 4 units total)

The picnic was cancelled due to an unexpected rainshower. (1 unit for cause)

After a few days of not speaking to each other, I finally wrote a letter to Yejin to apologize and to get along well again. (2 units for intentions, 6 units total)

Again, if the part describing causes or intentions include relevant specific information regarding what happened in the one-day period, count the units appropriately.

I was scolded by mother because I said a bad word I picked up that day at school.(1 cause including 3 units in it, 5 units total)

\section{j. Emotional responses}

If the response part contains emotions, or emotional responses that the respondent or other people experienced during the specific event, count the number of expressions describing emotions. These include both words that describe pure emotions, or other emotional responses, etc. The following are examples:

Happy, sad, get angry, get irritated, nervous, worried, feel good, feel bad, get excited, feel satisfied, hatred, remorse, liking, feel attracted to $\sim$, get tired, cry, have a tantrum, ... The following examples have multiple units.

I was confused and sad. (2 units)

Note: Do not take into account emotions outside 1-day time period relevant to the event.

My close friends and I went on a 2-day trip to Gangwon province and we virtually stayed up all night talking, playing games, having fun. I was so tired the next morning, but after I came back, I was really glad that I was able to go on that trip with my favorite friends.

In the answer above, the first day and night at the trip could be the 1-day period that is relevant for coding the level of details. Thus the part except that period is not being used for coding here, and the emotion of being glad is not counted in for that reason. Similarly, the following example mentions thoughts and emotional response that is experienced during the time of recalling the event, and thus should not be counted.

I had a big dispute with my best friend that made us grow apart since. Come to think of the matter, it started from a very trivial issue that we disagreed on, and Ifeel very sad about it. 